\title{
RATIONAL CHEREDNIK ALGEBRAS AND HILBERT SCHEMES II: REPRESENTATIONS AND SHEAVES
}

\author{
I. GORDON AND J. T. STAFFORD
}

\begin{abstract}
Let $H_{c}$ be the rational Cherednik algebra of type $A_{n-1}$ with spherical subalgebra $U_{c}=e H_{c} e$. Then $U_{c}$ is filtered by order of differential operators with associated graded ring gr $U_{c}=\mathbb{C}\left[\mathfrak{h} \oplus \mathfrak{h}^{*}\right]^{W}$, where $W$ is the $n$-th symmetric group. Using the $\mathbb{Z}$-algebra construction from [GS] it is also possible to associate to a filtered $H_{c^{-}}$or $U_{c^{-}}$module $M$ a coherent sheaf $\widehat{\Phi}(M)$ on the Hilbert scheme Hilb(n).

Using this technique, we study the representation theory of $U_{c}$ and $H_{c}$, and relate it to Hilb(n) and to the resolution of singularities $\tau: \operatorname{Hilb}(\mathrm{n}) \rightarrow \mathfrak{h} \oplus \mathfrak{h}^{*} / W$. For example, we prove:

- If $c=1 / n$, so that $L_{c}$ (triv) is the unique one-dimensional simple $H_{c}$-module, then $\widehat{\Phi}\left(e L_{c}(\right.$ triv $\left.)\right) \cong \mathcal{O}_{\mathrm{Z}_{\mathrm{n}}}$, where $\mathrm{Z}_{\mathrm{n}}=\tau^{-1}(0)$ is the punctual Hilbert scheme.

- If $c=1 / n+k$ for $k \in \mathbb{N}$ then, under a canonical filtration on the finite dimensional module $L_{c}$ (triv), gr $e L_{c}$ (triv) has a natural bigraded structure which coincides with that on $\mathrm{H}^{0}\left(\mathrm{Z}_{\mathrm{n}}, \mathcal{L}^{k}\right)$, where $\mathcal{L} \cong$ $\mathcal{O}_{\text {Hilb(n) }}(1)$; this confirms conjectures of Berest, Etingof and Ginzburg.

- Under mild restrictions on $c$, the characteristic cycle of $\widehat{\Phi}\left(e \Delta_{c}(\mu)\right)$ equals $\sum_{\lambda} K_{\mu \lambda}\left[Z_{\lambda}\right]$, where $K_{\mu \lambda}$ are Kostka numbers and the $Z_{\lambda}$ are (known) irreducible components of $\tau^{-1}(\mathfrak{h} / W)$.
\end{abstract}

\section{Contents}

1. Introduction 2

2. Graded and filtered modules for $\mathbb{Z}$-algebras $\quad 5$

3. Rational Cherednik algebras and Hilbert schemes 10

4. Representations of $H_{c}$ and coherent sheaves on Hilb(n) 17

5. Finite dimensional $U_{c}$-modules and their characteristic varieties 24

6. Characteristic cycles 29

7. Bimodules 39

8. A Conze embedding 43

Index of Notation $\quad 45$

$\begin{array}{ll}\text { References } & 46\end{array}$

1991 Mathematics Subject Classification. 14C05, 32S45,16S80, 16D90, 05E10.

Key words and phrases. Cherednik algebra, Hilbert scheme, category $\mathcal{O}$, characteristic varieties.

The first author was supported by the Nuffield Foundation Grant NAL/00625/A and the Leverhulme trust. He would like to thank the University of Washington and the University of California at Santa Barbara for their hospitality while parts of this paper were written. The second author was supported in part by the NSF grant DMS-0245320. 


\section{INTRODUCTION}

1.1. Let $c \in \mathbb{C}$. We assume throughout the paper that $c \notin \frac{1}{2}+\mathbb{Z}$ and, for simplicity, we will also assume in this introduction that $c \notin \mathbb{Q}_{\leq 0}$. The aim of this paper is to study the representation theory of the rational Cherednik algebra of type $A_{n-1}$, denoted $H_{c}$, and its spherical subalgebra $U_{c}=e H_{c} e$ and to relate $H_{c^{-}}$and $U_{c^{-}}$-modules to sheaves over the Hilbert scheme of points in the plane.

Glimpses of such a connection appear in [G2] and [BEG2] where it is shown that finite dimensional $H_{c^{-}}$ and $U_{c}$-modules deform the sections of some remarkable sheaves on Hilb(n). A more formal relationship is provided by the main theorem from the companion paper [GS]. In order to describe that result recall that both $H_{c}$ and $U_{c}$ can be filtered by degree of differential operators with associated graded rings gr $H_{c} \cong$ $\mathbb{C}\left[\mathfrak{h} \oplus \mathfrak{h}^{*}\right] * W$ and $\operatorname{gr} U_{c} \cong \mathbb{C}\left[\mathfrak{h} \oplus \mathfrak{h}^{*}\right]^{W}$, where $\mathfrak{h} \subset \mathbb{C}^{n}$ is the reflection representation of the $n^{\text {th }}$ symmetric group $W$; one can therefore regard $U_{c}$ as a deformation of $\mathbb{C}\left[\mathfrak{h} \oplus \mathfrak{h}^{*}\right]^{W}$. The theorem below provides a second way of passing to associated graded objects which shows that $U_{c}$ can also be regarded as a noncommutative deformation of a homogeneous coordinate ring of the Hilbert scheme Hilb(n) (this scheme provides a crepant resolution $\left.\tau: \operatorname{Hilb}(\mathrm{n}) \rightarrow \mathfrak{h} \oplus \mathfrak{h}^{*} / W\right)$.

1.2. Theorem. [GS, Theorem 1.4] There exists a filtered $\mathbb{Z}$-algebra B such that

(1) $U_{c}$-mod, the category of finitely generated $U_{c}$-modules, is equivalent to B-qgr, the quotient category of finitely generated graded B-modules modulo those of finite length;

(2) gr $B$, the associated graded ring of $B$, is isomorphic to (the $\mathbb{Z}$-algebra associated with) the homogeneous coordinate ring $\bigoplus_{k \geq 0} \mathrm{H}^{0}\left(\mathrm{Hilb}(\mathrm{n}), \mathcal{L}^{k}\right)$ for a certain ample line bundle $\mathcal{L}$ on $\mathrm{Hilb}(\mathrm{n})$.

The construction of $B$ is described in more detail in Subsection 3.5. In brief $B=\bigoplus_{i \geq j \geq 0} B_{i j}$, where $B_{i j}$ is the $\left(U_{c+i}, U_{c+j}\right)$-bimodule that provides a Morita equivalence between $U_{c+i}$ and $U_{c+j}$, and multiplication in $B$ is given in matrix fashion: $B_{i j} B_{j k} \subseteq B_{i k}$, but $B_{i j} B_{\ell k}=0$ if $j \neq \ell$. One should regard $B$ as being a little like a Rees ring, but in a situation where one may not have any proper ideals. Each $B_{i j}$ is filtered by order of differential operators, which induces the desired filtration $B=\bigcup \operatorname{ord}^{n} B$ on $B$.

1.3. Theorem 1.2 provides the following recipe for passing from a left $U_{c}$-module $M$ with a good filtration $\Lambda$ to a coherent sheaf $\Phi(M)=\Phi_{\Lambda}(M)$ on Hilb(n). Each $M(k)=B_{k 0} \otimes_{U_{c}} M$ has an induced tensor product filtration $\Lambda$ defined by $\Lambda^{n} M(k)=\sum_{i \leq n} \operatorname{ord}^{i} B_{k 0} \otimes \Lambda^{n-i} M$. This induces a filtration on $\widetilde{M}=B \otimes M$, again written $\Lambda$, whose associated graded object $\operatorname{gr}_{\Lambda} \widetilde{M}$ is a finitely generated gr $B$-module. Finally, by the theorem, $\operatorname{gr}_{\Lambda} \widetilde{M}$ corresponds to a coherent sheaf $\Phi(M)$ on $\operatorname{Hilb}(\mathrm{n})$.

This also works for any filtered $H_{c}$-module $(N, \Gamma)$ : use the induced tensor product filtration $\Lambda$ on $e H_{c} \otimes_{H_{c}} N$ to construct a sheaf $\widehat{\Phi}(N)=\Phi_{\Lambda}\left(e H_{c} \otimes N\right)$ on Hilb(n). Under our hypotheses on $c$, tensoring with $e H_{c}$ gives a Morita equivalence between $H_{c}$ and $U_{c}$ [GS, Theorem 3.3], so $\widehat{\Phi}$ is really the same construction as $\Phi$.

The basic technique of this paper is to exploit the functors $\Phi$ and $\widehat{\Phi}$ to better understand the representation theory of $U_{c}$ and $H_{c}$. 
1.4. This works particularly well for $\mathcal{O}_{c}$, the category of finitely generated $H_{c}$-modules on which $\mathbb{C}\left[\mathfrak{h}^{*}\right]$ acts locally nilpotently. The basic building blocks here are the standard modules $\Delta_{c}(\mu)$, where $\mu \in \operatorname{Irrep}(W)$ is an irreducible representation of $W$. These are analogues of Verma modules over a simple complex Lie algebra; in particular $\Delta_{c}(\mu) \cong \mathbb{C}[\mathfrak{h}] \otimes \mu$ as left $\mathbb{C}[\mathfrak{h}]$-modules. It follows from this that, for any good filtration $\Lambda$ on $e \Delta_{c}(\mu)$, the associated variety $\mathcal{V}\left(\operatorname{ann}_{\operatorname{gr} U_{c}}\left(\operatorname{gr}_{\Lambda} e \Delta_{c}(\mu)\right)\right)=\mathfrak{h} / W \subset \mathfrak{h} \oplus \mathfrak{h}^{*} / W$ is independent of $\mu$.

An interesting phenomenon occurs if we follow the procedure of (1.3) since the characteristic variety $\mathcal{V}\left(\operatorname{ann}\left(\operatorname{gr}_{\Lambda} \widetilde{e \Delta_{c}(\mu)}\right)\right)$ of $\widetilde{e \Delta_{c}(\mu)}$ is a subvariety of Hilb(n) that does depend upon $\mu$. We will state the result using a finer invariant called the characteristic cycle, $\mathbf{C h}\left(\Delta_{c}(\mu)\right)$, which counts the irreducible components of the characteristic variety of $\widehat{\Phi}\left(\Delta_{c}(\mu)\right)$ in Hilb(n) with multiplicities (see (2.7) for the formal definition).

Theorem. (Theorem 6.7) Let $\Delta_{c}(\mu)$ be the standard $H_{c}$-module corresponding to $\mu \in \operatorname{Irrep}(W)$. Then

$$
\mathbf{C h}\left(\Delta_{c}(\mu)\right)=\sum_{\lambda} K_{\mu \lambda}\left[Z_{\lambda}\right]
$$

where $K_{\mu \lambda}$ are Kostka numbers as defined in (3.11) and the $Z_{\lambda}$ 's are the irreducible components of $Z=$ $\tau^{-1}(\mathfrak{h} / W)$ described in (6.4).

When $n=2$ and $c=1 / 2$ an easy direct proof of this and other theorems is given in (6.12-6.14) which the reader may like to use as an introduction to the results of this paper.

1.5. The above theorem illustrates a fact that will appear repeatedly in this paper: important modules $M$ over $H_{c}$ or $U_{c}$ correspond naturally to important sheaves and combinatorial data on Hilb(n).

One reason why Theorem 1.2 and the functors $\Phi$ and $\widehat{\Phi}$ provide a bridge between Hilbert schemes and Cherednik algebras is that the construction of $B$ carries key elements from both theories. For instance, the shift functors $\left(B_{j+1, j} \otimes-\right)$ are fundamental to the theory of Cherednik algebras; they are also the analogue of the endo-functor $(\mathcal{L} \otimes-)$ in $\operatorname{Coh}(\operatorname{Hilb}(\mathrm{n}))$ : given a $U_{c}$-module $M$ with a good filtration $\Lambda$, we show that $\mathcal{L} \otimes \Phi_{\Lambda}(M)=\Phi_{\Gamma}\left(B_{c+1, c} \otimes M\right)$ for the appropriate filtration $\Gamma$ (see Lemma 4.4). As Proposition 1.7 and Theorem 1.8 illustrate, this is important since it enables one to study whole families of modules simultaneously.

1.6. A consequence of Theorem 1.4 is that the Grothendieck group $K\left(\mathcal{O}_{c}\right)$ is naturally isomorphic to the top degree Borel-Moore homology group $H_{2 n-2}(Z, \mathbb{C}$ ) (see Corollary 6.10). If [GGOR, Remark 5.17] is confirmed, ${ }^{1}$ this can be considerably refined: the characteristic cycles of the simple modules in $\mathcal{O}_{c}$ give a canonical basis of $H_{2 n-2}(Z, \mathbb{C})$ which corresponds to Leclerc and Thibon's lower canonical basis of the ring of symmetric functions. The details can be found in Subsection 6.11.

\footnotetext{
${ }^{1}$ This has been announced recently by Rouquier.
} 
1.7. The category $\mathcal{O}_{c}$ also includes the finite dimensional $H_{c}$-modules: they occur precisely when $c=r / n$ for some $r \in \mathbb{N}$ with $(r, n)=1$. In such a case $H_{c}$ has a unique finite dimensional simple module $L_{c}$ (triv) (see (5.2) for the notation) and every finite dimensional $H_{c}$-module is a direct sum of copies of $L_{c}$ (triv). We note that $L_{1 / n}$ (triv) is one-dimensional and, by [GS, Proposition 3.16], that $e L_{k+1 / n}\left(\right.$ triv) $\cong B_{k 0} \otimes_{U_{1 / n}} e L_{1 / n}$ (triv), for $k \in \mathbb{N}$.

Proposition. (Proposition 5.4) Give $L_{1 / n}\left(\right.$ triv) the trivial filtration $\Lambda^{0}=L_{1 / n}($ triv $)$. Then $\widehat{\Phi}\left(L_{1 / n}(\right.$ triv $\left.)\right) \cong$ $\mathcal{O}_{\mathrm{Z}_{\mathrm{n}}}$, where $\mathrm{Z}_{\mathrm{n}}=\tau^{-1}(\mathbf{0})$ is the punctual Hilbert scheme.

1.8. Let $c=k+1 / n$ and give $e L_{c}$ (triv) $=B_{k 0} \otimes e L_{1 / n}$ (triv) the tensor product filtration induced from the trivial filtration on $e L_{1 / n}$ (triv). Then gr $e L_{c}$ (triv) and (under an analogous filtration) gr $L_{c}$ (triv) are bigraded: one component of this grading is induced from the Euler grading on differential operators; the other comes from the fact that we have taken an associated graded module. Similarly, $\widehat{\Phi}\left(L_{c}\right.$ (triv)) is a $\left(\mathbb{C}^{*}\right)^{2}$-equivariant sheaf on Hilb(n). Using Proposition 1.7 we are able to identify this sheaf, and we can then describe the corresponding bigraded characters of gr $L_{c}$ (triv) and gr $e L_{c}$ (triv). Modulo a technical change in the filtrations, this gives the following result, the first part of which confirms conjectures of Berest, Etingof and Ginzburg, [BEG2, Conjectures 7.2 and 7.3].

Theorem. (Theorem 5.11 and (5.12)) Let $c=k+1 / n$ for some $k \in \mathbb{N}$. Then there are natural filtrations $\Lambda$ on $e L_{c}\left(\right.$ triv) and $L_{c}($ triv) such that

(1) there are bigraded isomorphisms $\operatorname{gr}_{\Lambda} L_{c}($ triv $) \cong \mathrm{H}^{0}\left(\mathrm{Z}_{\mathrm{n}}, \mathcal{P} \otimes \mathcal{L}^{k-1}\right)$ and $\operatorname{gr}_{\Lambda} e L_{c}($ triv $) \cong \mathrm{H}^{0}\left(\mathrm{Z}_{\mathrm{n}}, \mathcal{L}^{k}\right)$;

(2) under this bigrading, $\operatorname{gr}_{\Lambda} e L_{c}$ (triv) has Poincaré series equal to the $(t, q)$-Catalan-like number defined by [GH1, (3.26)].

1.9. A further example of an $H_{c}$-module corresponding to an important sheaf is given by the module $H_{c}$ itself. Under the order filtration ord of differential operators, Theorem 4.5 shows that $\widehat{\Phi}_{\text {ord }}\left(H_{c}\right) \cong \mathcal{P}$, the Procesi bundle on Hilb(n). This is a remarkable rank $n$ ! bundle that arises from Haiman's isospectral Hilbert scheme. See (3.7) or [Ha2] for more details.

1.10. There is a second construction that produces a coherent sheaf on Hilb(n) from an $H_{c}$-module $N$ with a good filtration $\Gamma$, and which provides a potential connection between our $\mathbb{Z}$-algebra construction and the Fourier-Mukai equivalences of Bridgeland-King-Reid. The construction is described as follows. Theorems of Bridgeland-King-Reid and Haiman provide an equivalence of categories $D\left(\mathbb{C}\left[\mathfrak{h} \oplus \mathfrak{h}^{*}\right] * W\right) \stackrel{\sim}{\rightarrow} D(\operatorname{Hilb}(\mathrm{n}))$ from the bounded derived category of finitely generated $\mathbb{C}\left[\mathfrak{h} \oplus \mathfrak{h}^{*}\right] * W$-modules to that of coherent sheaves on Hilb(n). Applying this equivalence to the finitely generated $\mathbb{C}\left[\mathfrak{h} \oplus \mathfrak{h}^{*}\right] * W$-module $\operatorname{gr}_{\Gamma} N$, regarded as a complex concentrated in degree zero, gives a bounded complex $\Psi(N)=\Psi_{\Gamma}(N)$ of coherent sheaves on $\operatorname{Hilb}(\mathrm{n})$.

In all the examples we have calculated, the complex $\Psi(N)$ is quasi-isomorphic to $\widehat{\Phi}(N)$. We ask whether this is true in general; we are only able to show that there is a surjective mapping from the zeroth cohomology 
sheaf $\Psi^{0}(N)$ onto $\widehat{\Phi}(N)$, see Proposition 4.11. If this question had a positive answer it would imply that all higher cohomology of $\mathcal{P}^{*} \otimes \widehat{\Phi}(N)$ must vanish. A vanishing theorem in this spirit, which has consequences for symmetric function theory, has been proposed in [Ha3, Conjecture 3.2], see (4.12).

1.11. Our final application is an analogue of the Conze embedding [Co] for $U_{c}$. The Dunkl-Cherednik map [EG, Proposition 4.5] provides an inclusion $U_{c} \hookrightarrow e\left(D\left(\mathfrak{h}^{\text {reg }}\right) * W\right) e \cong D\left(\mathfrak{h}^{\text {reg }}\right)^{W}$ and it is straightforward to see that this actually embeds $U_{c}$ into the Weyl algebra $D(\mathfrak{h} / W)$. Our analogue of the Conze embedding gives a precise description of the $U_{c}$-module structure of this algebra:

Proposition. (Proposition 8.5) Under the natural identifications, $D(\mathfrak{h} / W)=\bigcup_{k \geq 0} B_{k 0}^{*}$, where the $B_{k 0}^{*}=$ $\operatorname{Hom}_{U_{c}}\left(B_{k 0}, U_{c}\right)$ are projective left $U_{c}$-modules. Thus $D(\mathfrak{h} / W)$ is a flat left $U_{c}$-module.

Once again, this corresponds to a fact about Hilbert schemes: in this case it is the result from [Ha1, Section 2] that shows that the subset $U_{\left(1^{n}\right)}$ of $\mathrm{Hilb}(\mathrm{n})$ is isomorphic to the affine space $\mathbb{A}^{2 n-2}$.

1.12. Plan. The paper is organised as follows. In Section 2 we study filtered $\mathbb{Z}$-algebras and their modules and show that classical facts concerning characteristic varieties and characteristic cycles for filtered noetherian algebras do generalise to $\mathbb{Z}$-algebras. Section 3 provides some background material while Section 4 sees the construction of the functors $\Phi$ and $\widehat{\Phi}$ and a discussion of their relationship to the equivalences of BridgelandKing-Reid. In Section 5 we study the effect of $\Phi$ and $\widehat{\Phi}$ on the finite dimensional simple $U_{c^{-}}$and $H_{c^{-}}$modules, in particular using this to determine their bigraded characters. Our results on characteristic cycles for $\mathcal{O}_{c}$ are presented in Section 6, whilst analogous results for Harish-Chandra modules are proved in Section 7 and the Conze embedding is constructed in Section 8.

\section{Graded And filtered modules for $\mathbb{Z}$-ALGebras}

2.1. Throughout this paper a $\mathbb{Z}$-algebra will mean a lower triangular $\mathbb{Z}$-algebra. By definition, this is a (non-unital) algebra $B=\bigoplus_{i \geq j \geq 0} B_{i j}$, where multiplication is defined in matrix fashion: $B_{i j} B_{j k} \subseteq B_{i k}$ for $i \geq j \geq k \geq 0$ but $B_{i j} B_{\ell k}=0$ if $j \neq \ell$. Although $B$ cannot have a unit element, we do require that each subalgebra $B_{i i}$ has a unit element $1_{i}$ such that $1_{i} b_{i j}=b_{i j}=b_{i j} 1_{j}$, for all $b_{i j} \in B_{i j}$.

Let $B$ be a $\mathbb{Z}$-algebra. We define the category $B$-Grmod to be the category of $\mathbb{N}$-graded left $B$-modules $M=\bigoplus_{i \in \mathbb{N}} M_{i}$ such that $B_{i j} M_{j} \subseteq M_{i}$ for all $i \geq j$ and $B_{i j} M_{k}=0$ if $k \neq j$. Homomorphisms are defined to be graded homomorphisms of degree zero. The subcategory of noetherian graded left $B$-modules will be denoted $B$-grmod. In all examples considered in this paper $B$-grmod will consist precisely of the finitely generated graded left $B$-modules.

A module $M \in B$-Grmod is bounded if $M_{n}=0$ for all but finitely many $n \in \mathbb{Z}$ and torsion if it is a direct limit of bounded modules. We denote the category of torsion modules by $B$-Tors. The corresponding subcategory of $B$-grmod is denoted $B$-tors; thus $B$-tors just consists of bounded, noetherian modules. Finally, 
in order to obtain geometric categories, we construct the quotient categories $B$-Qgr $=B$-Grmod $/ B$-Tors and $B$-qgr $=B$-grmod $/ B$-tors. We write $\pi(M)$ for the image in $B$-Qgr of $M \in B$-Grmod.

2.2. We will be interested in two types of examples of $\mathbb{Z}$-algebras.

First, suppose that $S=\bigoplus_{n \geq 0} S_{n}$ is a noetherian $\mathbb{N}$-graded algebra. To $S$ we associate a $\mathbb{Z}$-algebra $\widehat{S}=\bigoplus_{i \geq j \geq 0} \widehat{S}_{i j}$ by setting $\widehat{S}_{i j}=S_{i-j}$ with multiplication induced from that in $S$. Define categories $S$-Grmod, $\ldots, S$-qgr in the usual manner. In particular, $S$-Grmod denotes the category of $\mathbb{Z}$-graded $S$-modules, from which the other definitions follow as in the last paragraph. As explained in [GS, (5.3)] we have equivalences $S$-Qgr $\simeq \widehat{S}$-Qgr and $S$-qgr $\simeq \widehat{S}$-qgr. If $S$ is commutative and generated as an $S_{0}$-algebra by $S_{1}$, then $S$-qgr and hence $\widehat{S}$-qgr are equivalent to $\operatorname{Coh} \operatorname{Proj}(S)$, the category of $\operatorname{coherent} \operatorname{sheaves}$ on $\operatorname{Proj}(S)$ [EGA, Proposition 3.3.5].

Second, suppose that $\left\{R_{n}: n \in \mathbb{N}\right\}$ are Morita equivalent noetherian algebras, with the equivalence induced from the progenerative $\left(R_{n+1}, R_{n}\right)$-bimodules $P_{n}$. Define $R_{i j}=P_{i-1} \otimes_{R_{i-1}} \otimes \cdots \otimes_{R_{j+2}} P_{j+1} \otimes_{R_{j+1}} P_{j}$ and $R_{j j}=R_{j}$, for $i>j \geq 0$. The corresponding $\mathbb{Z}$-algebra $R_{\mathbb{Z}}=\bigoplus_{i \geq j \geq 0} R_{i j}$ will be called the $M o r i t a \mathbb{Z}$ -

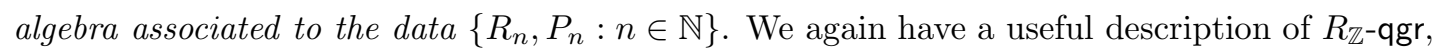

Lemma. [GS, Lemma 5.5] Let $R_{\mathbb{Z}}$ be a Morita $\mathbb{Z}$-algebra as above. Then

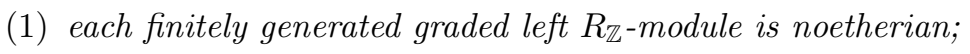

(2) the association $\phi: M \mapsto \bigoplus_{n \in \mathbb{N}} R_{n 0} \otimes_{R_{0}} M$ induces an equivalence of categories $R_{0}$-mod $\stackrel{\sim}{\longrightarrow} R_{\mathbb{Z}}$-qgr.

2.3. Associated graded techniques. A number standard constructions for unital noetherian rings extend routinely to $\mathbb{Z}$-algebras: those that will be useful in this paper are the notions of associated graded modules and characteristic cycles.

In order for filtrations of a $\mathbb{Z}$-algebra $R$ to work properly, the filtrations on the various components $R_{i j}$ need to be compatible. The abstract requirements for this are quite lengthy and so we will assume that we are in the following special situation.

(1) $R=R_{\mathbb{Z}}=\bigoplus_{i \geq j \geq 0} R_{i j}$ is the Morita $\mathbb{Z}$-algebra associated to the data $\left\{R_{n}, P_{n}: n \in \mathbb{N}\right\}$.

(2) Each $R_{i}$ is a noetherian domain, and each $P_{i}$ has rank one (on one side and therefore the other). As a result, we may identify each $R_{n+1}=\operatorname{End}_{R_{n}}\left(P_{n}\right)$ with a subring of the quotient division ring, say $D$, of $R_{0}$.

(3) There is a ring $E \subset D$ containing each $R_{i}$ and $P_{i}$ such that $E$ has an ascending $\mathbb{N}$-filtration $F^{k} E$ with a commutative associated graded ring $\operatorname{gr}_{F} E$.

The point of these assumptions is that we can take the induced filtrations $\left\{F^{k} R_{i j}\right\}$ on the $R_{i j}$ and hence on $R$ itself. It is then routine to check that this filtration is compatible with the ring structure of $R$ and so we obtain an associated graded $\mathbb{Z}$-algebra

$$
\operatorname{gr}_{F} R=\bigoplus_{i \geq j \geq 0} \operatorname{gr}_{F} R_{i j}, \quad \text { where } \quad \operatorname{gr}_{F} R_{i j}=\bigoplus_{n \in \mathbb{N}} \frac{F^{n} R_{i j}}{F^{n-1} R_{i j}}
$$


The final assumption that we will make is:

(4) $\operatorname{gr}_{F} R \cong \widehat{S}$, the $\mathbb{Z}$-algebra associated to some finitely generated commutative graded domain $S$.

Conditions (1)-(3) occur reasonably frequently. One case is given by taking the $R_{i}=U_{c+i}($ see $(3.5))$, but one can also take appropriate factor rings of the enveloping $U(\mathfrak{g})$ of a semisimple Lie algebra $\mathfrak{g}$, where the progenerators arise from translation functors (see, for example, [JS]). Condition (4) is more stringent, although Theorem 1.2(2) shows that it does apply when $R_{i}=U_{c+i}$.

2.4. Keep hypotheses (1)-(3) of (2.3) and let $M=\bigcup \Lambda^{i} M$ be a filtered left $R_{0}$-module. Each module $M(i)=R_{i 0} \otimes_{R_{0}} M$ is then filtered by the tensor product filtration

$$
\Lambda^{k} M(i)=\sum_{\ell \in \mathbb{Z}} F^{\ell} R_{i 0} \otimes \Lambda^{k-\ell} M
$$

We therefore obtain an associated graded $\operatorname{gr}_{F} R_{i}$-module $\operatorname{gr}_{\Lambda} M(i)=\bigoplus \Lambda^{k} M(i) / \Lambda^{k-1} M(i)$. Summing over all $i$ gives an associated graded $\operatorname{gr}_{F} R$-module $\operatorname{gr}_{\Lambda} \widetilde{M}=\bigoplus \operatorname{gr}_{\Lambda} M(i)$ for the $R$-module $\widetilde{M}=\bigoplus_{i \in \mathbb{Z}} M(i)$. When Hypothesis 2.3(4) holds we can and will $\operatorname{regard} \operatorname{gr}_{\Lambda} M$ as a graded $S$-module.

We say that $\Lambda$ is a good filtration on $M$ if $\operatorname{gr}_{\Lambda} M$ is a finitely generated $\operatorname{gr}_{F} R_{0}$-module. Similarly, a filtration $\Lambda$ on $\widetilde{M}$ is good if $\operatorname{gr}_{\Lambda} \widetilde{M}$ is a finitely generated $\operatorname{gr}_{F} R$-module. If $m \in \Lambda^{i} M \backslash \Lambda^{i-1} M$, we write $\sigma(m)=\left[m+\Lambda^{i-1} M\right] \in \operatorname{gr}_{\Lambda} M$ for the principal symbol of $m$.

2.5. Lemma. Keep Hypotheses (1)-(3) of (2.3) and let $M$ be a left $R_{0}$-module.

(1) If $\Lambda$ is a good filtration on $M$ then the induced filtration $\Lambda$ on $\widetilde{M}$ is good.

(2) Given two good filtrations $\Lambda$ and $\Gamma$ on $M$, there exists a positive integer $t$ such that, for all $n$ and $k$,

$$
\Lambda^{n} M(k) \subseteq \Gamma^{n+t} M(k) \subseteq \Lambda^{n+2 t} M(k)
$$

(3) Assume that Hypothesis (2.3)(4) also holds and regard $\operatorname{gr}_{\Lambda} \widetilde{M}$ as an object in S-grmod. Define the associated radical ideal $N=N_{\widetilde{M}}$ of $\widetilde{M}$ to be the radical ideal $N=\sqrt{I}$ of $I=\operatorname{ann}_{S}\left(\operatorname{gr}_{\Lambda} \widetilde{M}\right)$. Then $N$ is independent of the choice of good filtration $\Lambda$.

Proof. (1) Since the filtration $\Lambda$ on $M$ is good, we may pick generators $m_{i}$ for $M$ with $m_{i} \in \Lambda^{j_{i}} M \backslash \Lambda^{j_{i}-1} M$ such that the principal symbols $\sigma\left(m_{i}\right)$ generate $\operatorname{gr}_{\Lambda} M$. Pick a free $R_{0}$-module $G=\bigoplus R_{0} g_{i}$ and filter $G$ by putting $g_{i}$ in degree $j_{i}$; thus $G$ is filtered by $\Phi^{n} G=\bigoplus\left(F^{n-j_{i}} R_{0}\right) g_{i}$. Then the natural surjection $\alpha: G \rightarrow M$ given by $g_{i} \mapsto m_{i}$ is filtered surjective in the sense that, for each $n$, we have $\alpha\left(\Phi^{n} G\right)=\Lambda^{n} M$. Equivalently, $\alpha$ induces a surjection $\operatorname{gr} \alpha: \operatorname{gr}_{\Phi} G \rightarrow \operatorname{gr}_{\Lambda} M$.

Now consider the induced map $G(k)=R_{k 0} \otimes_{R_{0}} G \rightarrow M(k)$. The tensor product filtration (2.4.1) on $G(k)$ induces a surjection $\alpha_{k}: \Phi^{n} G(k) \rightarrow \Lambda^{n} M(k)$ for all $n$ and $k$ and hence a surjection of graded groups $\operatorname{gr} \alpha_{k}: \operatorname{gr}_{\Phi} G(k) \rightarrow \operatorname{gr}_{\Lambda} M(k)$. Since $G$ is free, we have, for each $n$ and $k$,

$$
\Phi^{n} G(k)=\sum_{j} F^{n-j} R_{k 0} \otimes \Phi^{j} G=\sum_{j, t} F^{n-j} R_{k 0} \otimes\left(F^{j-j_{t}} R_{0}\right) g_{t}=\sum_{t}\left(F^{n-j_{t}} R_{k 0}\right) \otimes g_{t} .
$$


The filtration $\Phi G(k)$ is therefore the natural filtration on the (weighted) direct sum of copies of $R_{k 0}$ and so the associated graded module $\operatorname{gr}_{\Phi} \widetilde{G}$ is just the weighted sum $E=\bigoplus_{t} S\left[j_{t}\right]$. Since $g r_{\Lambda} \widetilde{M}$ is a homomorphic image of $E$, it is finitely generated.

(2) By [KL, Corollary 6.12] the desired equation holds for $k=0$. By (2.4.1) it then holds for each $k$, with the same value of $t$.

(3) By using intermediary filtrations, as in [MR, Lemma 8.6.11], we may assume in part (2) that $t=1$ for $k=0$ and hence for all $k$. As in the proof of [MR, Lemma 8.6.12], we then obtain short exact sequences

$$
0 \rightarrow Z \rightarrow \operatorname{gr}_{\Lambda}(\widetilde{M}) \rightarrow Y \rightarrow 0 \quad \text { and } \quad 0 \rightarrow Y \rightarrow \operatorname{gr}_{\Gamma}(\widetilde{M}) \rightarrow Z[1] \rightarrow 0,
$$

where $Y=\bigoplus_{k} \bigoplus_{n} \Lambda^{n+1} M(k) / \Gamma^{n} M(k)$ and $Z=\bigoplus_{k} \bigoplus_{n} \Gamma^{n} M(k) / \Lambda^{n} M(k)$. If $J=\operatorname{ann}_{S}\left(\operatorname{gr}_{\Gamma} \widetilde{M}\right)$, then it follows that $I^{2} \subseteq J$ and $J^{2} \subseteq I$.

2.6. Definition. Keep the hypotheses of Lemma 2.5(3). Then $N_{\widetilde{M}}$ is a graded ideal of $S$ and we define the characteristic variety $\operatorname{Char}(M)$ of $M$ to be the projective subvariety $\mathcal{V}\left(N_{\widetilde{M}}\right)$ of $\operatorname{Proj}(S)$ defined by $N_{\widetilde{M}}$. The lemma ensures that this subvariety is independent of the choice of good filtration $\Lambda$, and so it is an invariant of $M$. A second variety associated to $M$ is

$$
\operatorname{Char}_{0}(M)=\mathcal{V}\left(\operatorname{ann}_{\operatorname{gr} R_{0}}\left(\operatorname{gr}_{\Lambda} M\right)\right) \subseteq \operatorname{Spec}\left(\operatorname{gr} R_{0}\right)
$$

In order to avoid any possible confusion with Char $M$ we will always call $\operatorname{Char}_{0} M$ the associated variety of $M$, as it is sometimes termed in the literature.

2.7. Characteristic cycles. In the classical situation of filtered noetherian rings it is often useful to refine the characteristic variety of a module to the characteristic cycle; see, for example, [Bj2, Remark 5.7]. As we show next, this also works for $\mathbb{Z}$-algebras.

Keep the hypotheses and notation of Lemma 2.5, and write Min $\widetilde{M}$ for the set of prime ideals minimal over the associated radical ideal $N_{\widetilde{M}}$. If $P \in \operatorname{Min} \widetilde{M}$, we define $n_{\widetilde{M}, P}$ to be the length of the (necessarily finite length) $S_{P}$-module $M_{P}$. We note that if $S_{[P]}=S\left[\mathcal{C}^{-1}\right]_{0}$, where $\mathcal{C}$ denotes the set of homogeneous elements in $S \backslash P$, then $n_{\widetilde{M}, P}$ also equals the length of the $S_{[P]}-$ module $\operatorname{gr}_{\Lambda}(\widetilde{M}) S\left[\mathcal{C}^{-1}\right]_{0}$. The characteristic cycle $\mathbf{C h} M$ is defined to be

$$
\text { Ch } M=\sum_{\operatorname{Min} \widetilde{M}} n_{\widetilde{M}, P} \mathcal{V}(P)
$$

Clearly this is a finite sum with finite coefficients. By the next result it is also a well-defined notion. We remark that the more usual associated cycle of $\operatorname{gr}_{\Lambda} M$ will not be used in this paper and so there should be no confusion in our notation.

Lemma. Keep the hypotheses (1-4) of (2.3) and let $M$ be a left $R_{0}$-module. Then the characteristic cycle Ch $M$ is independent of the choice of the good filtration $\Lambda$ used in its definition. 
Proof. Suppose that the module $M$ has two good filtrations $\Lambda$ and $\Gamma$ and, for the moment, write $\mathbf{C h}_{\Lambda} M$ for the characteristic cycle of $M$ induced from $\Lambda$. As in the proof of Lemma 2.5(3) we may assume that $t=1$ in the conclusion of part (2) of that lemma, and hence that we have the two short exact sequences (2.5.1). It follows immediately from those sequences that $\left(\operatorname{gr}_{\Lambda} \widetilde{M}\right)_{P}$ and $\left(\operatorname{gr}_{\Gamma} \widetilde{M}\right)_{P}$ have the same (finite) length for any $P \in \operatorname{Min} \widetilde{M}$. In other words, $\mathbf{C h}_{\Lambda} M=\mathbf{C h}_{\Gamma} M$.

2.8. Assume that $R_{0}$ satisfies hypotheses (1-4) of (2.3). We will need to understand how characteristic cycles relate to short exact sequences of $R_{0}$-modules. In general one does not obtain additivity on such sequences since embedded primes cause problems - a standard example is given by the short exact sequence of $\mathbb{C}[x]$-modules $0 \rightarrow(x) \rightarrow \mathbb{C}[x] \rightarrow \mathbb{C} \rightarrow 0$. The standard way around this is to only look at the components of the characteristic variety of maximal dimension and we do the same here.

Thus, if $M$ is a finitely generated filtered $R_{0}$-module, write $\operatorname{Min}^{\prime} \widetilde{M}$ for the subset of $\operatorname{Min} \widetilde{M}$ consisting of those prime ideals $P$ for which $\operatorname{dim} \mathcal{V}(P)=\operatorname{dim} \mathcal{V}\left(N_{\widetilde{M}}\right)$ and let $\mathbf{r C h} M$ be the corresponding restricted characteristic cycle:

$$
\operatorname{rCh} M=\sum_{\operatorname{Min}^{\prime} \widetilde{M}} n_{\widetilde{M}, P} \mathcal{V}(P)
$$

By Lemma 2.7 this too is well-defined.

Lemma. Assume that $R_{0}$ satisfies hypotheses (1-4) of (2.3) and let $0 \rightarrow A \rightarrow B \stackrel{\beta}{\rightarrow} C \rightarrow 0$ be a short exact sequence of finitely generated left $R_{0}$-modules. Then precisely one of the following cases occurs:

(a) $\operatorname{dim} \operatorname{Char}(A)<\operatorname{dim} \operatorname{Char}(B)=\operatorname{dim} \operatorname{Char}(C)$ and $\mathbf{r C h} B=\mathbf{r C h} C$ ；

(b) $\operatorname{dim} \operatorname{Char}(A)=\operatorname{dim} \operatorname{Char}(B)>\operatorname{dim} \operatorname{Char}(C)$ and $\mathbf{r C h} B=\mathbf{r C h} A$;

(c) $\operatorname{dim} \operatorname{Char}(A)=\operatorname{dim} \operatorname{Char}(B)=\operatorname{dim} \operatorname{Char}(C)$ and $\mathbf{r C h} B=\mathbf{r C h} A+\mathbf{r C h} C$.

Proof. Choose a good filtration $\Gamma$ on $B$ and give $A$ and $C$ the induced filtrations $\Gamma^{t} A=\Gamma^{t} B \cap A$ and $\Gamma^{t} C=\beta\left(\Gamma^{t} B\right)$. It is an easy and standard exercise to check that this induces a short exact sequence of gr $R_{0^{-}}$ modules and that the analogue of the lemma holds for the restricted characteristic cycles of those graded modules (see, for example, [Bj2, Formula 5.8]). Unfortunately the induced tensor product filtration on $\widetilde{A}$, etc, need not be filtered exact and so one will not necessarily obtain a short exact sequence of associated graded modules. So the proof needs to be a little more involved.

In order to simplify the notation, we want to assume that $S$ is generated in degree one, which is easy to arrange. By [EGA, Lemme 2.1.6(v) and Proposition 2.4.7(i)], some Veronese ring $S^{(n)}=\bigoplus S_{i n}$ is generated by $S_{0}^{(n)}=S_{0}$ and $S_{1}^{(n)}=S_{n}$ while $\operatorname{Proj}(S)=\operatorname{Proj}\left(S^{(n)}\right)$ for any such $n$. Thus if we pass to the Veronese $\mathbb{Z}$-algebra $R^{(n)}=\bigoplus R_{i n, j n}$, with associated graded $\operatorname{ring} \operatorname{gr}_{F} R^{(n)}=\widehat{S^{(n)}}$, then the associated cycle of $R^{(n)} \otimes_{R_{0}} B$ will equal that of $\widetilde{B}$. Thus we may assume that $S$ is generated in degree one.

Consider the induced short exact sequence $0 \rightarrow \widetilde{A} \rightarrow \widetilde{B} \stackrel{\widetilde{\beta}}{\rightarrow} \widetilde{C} \rightarrow 0$. Give $\widetilde{B}$ the tensor product filtration $\Gamma$, which it is convenient to write as $\widetilde{\Gamma}$. Give $\widetilde{A}$ the induced filtration $\widetilde{\Gamma}$; thus $\widetilde{\Gamma}^{m} A(k)=A(k) \cap \widetilde{\Gamma}^{m} B(k)$ for 
all $m, k$. It is unnecessary to do this with $\widetilde{C}$ since, as in the proof of Lemma $2.5(1), \Gamma^{m} C(k)=\widetilde{\beta}\left(\widetilde{\Gamma}^{m} B(k)\right)$ for all $m, k$. It is immediate that $0 \rightarrow \widetilde{\Gamma}^{m} \widetilde{A} \rightarrow \widetilde{\Gamma}^{m} \widetilde{B} \rightarrow \Gamma^{m} \widetilde{C} \rightarrow 0$ is exact for each $m$ and hence that the associated complex of graded modules

$$
0 \rightarrow \operatorname{gr}_{\widetilde{\Gamma}} \widetilde{A} \rightarrow \operatorname{gr}_{\widetilde{\Gamma}} \widetilde{B} \rightarrow \operatorname{gr}_{\Gamma} \widetilde{C} \rightarrow 0
$$

is exact.

By Lemma $2.5(1), \operatorname{gr}_{\widetilde{\Gamma}} \widetilde{B}$ is finitely generated and therefore, by Hypothesis $2.3(4)$, so is $\operatorname{gr}_{\widetilde{\Gamma}} \widetilde{A}$. Pick an integer $t$ such that $\operatorname{gr}_{\widetilde{\Gamma}} \widetilde{A}$ is generated by $\bigoplus_{j \leq t} \operatorname{gr}_{\widetilde{\Gamma}} A(j)$. Define a new filtration $\Lambda$ on $\widetilde{A}_{\geq t}=\bigoplus_{i \geq t} A(i)$ by defining $\Lambda=\widetilde{\Gamma}$ on $A(t)$ and the tensor product filtration thereafter; thus $\Lambda^{m} A(i)=\sum_{j} F^{j} R_{i t} \otimes \widetilde{\Gamma}^{m-j} A(t)$, for $i>t$. The choice of $t$ and the fact that $S$ is generated in degree one ensures that

$$
\operatorname{gr}_{\widetilde{\Gamma}} A(m)=\sum_{j \leq t} S_{m-j} \operatorname{gr}_{\widetilde{\Gamma}} A(j)=S_{m-t} \operatorname{gr}_{\Lambda} A(t),
$$

for all $m \geq t$. Pulling this back to $\widetilde{A}_{\geq t}$ gives

$$
\widetilde{\Gamma}^{j} A(m)=\sum_{v \geq 0} F^{v} R_{m t} \otimes \Lambda^{j-v}\left(R_{t 0} \otimes A\right)=\Lambda^{j} A(m),
$$

for any $m \geq t$ and $j \geq 0$. In other words, $\Lambda=\widetilde{\Gamma}$.

Now consider characteristic cycles. Under the tensor product filtration $\operatorname{gr}_{\Gamma} \widetilde{B}$ and $\operatorname{gr}_{\Gamma} \widetilde{B}_{\geq t}$ only differ in the first $t$ terms and so in $\operatorname{Proj}(S)$ they have the same characteristic variety and restricted characteristic cycle. So we may work with $\widetilde{B}_{\geq t}$. But now the choice of good filtration on $A(t)$ is irrelevant, so we may choose the filtration $\Lambda=\widetilde{\Gamma}$ on $A(t)$ and the original filtrations $\widetilde{\Gamma}=\Gamma$ on $B(t)$ and $C(t)$. By (2.8.2) we therefore obtain a short exact sequence

$$
0 \rightarrow \operatorname{gr}_{\Lambda} \widetilde{A}_{\geq t} \rightarrow \operatorname{gr}_{\Gamma} \widetilde{B}_{\geq t} \rightarrow \operatorname{gr}_{\Gamma} \widetilde{C}_{\geq t} \rightarrow 0,
$$

for which the restricted characteristic cycles of the three terms are $\mathbf{r C h} A$, respectively $\mathbf{r C h} B$ and $\mathbf{r C h} C$.

It is now routine to see that the conclusion of the lemma is satisfied. Indeed, if $P \in \operatorname{Min}^{\prime} \widetilde{B}$, then localising (2.8.3) at $P$ gives a short exact sequence of finite dimensional $S_{P}$-modules and so the dimensions add: $n_{\widetilde{B}, P}=n_{\widetilde{A}, P}+n_{\widetilde{C}, P}$.

\section{Rational Cherednik algebras and Hilbert schemes}

In this section we collect some of the basic material we need concerning rational Cherednik algebras and Hilbert schemes.

3.1. The rational Cherednik algebra of type $A$. Let $W=\mathfrak{S}_{n}$ be the symmetric group on $n$ letters, regarded as the Weyl group of type $A_{n-1}$ acting on its $(n-1)$-dimensional reflection representation $\mathfrak{h} \subset \mathbb{C}^{n}$ by permutations. We will always identify $\mathfrak{h}^{*}$ with its image in the coordinate ring $\mathbb{C}[\mathfrak{h}]$ and we fix a basis $\left\{x_{i}\right\}$ of $\mathfrak{h}^{*}$. Let $\mathcal{S}=\{s=(i, j)$ with $i<j\} \subset W$ denote the reflections, with reflecting hyperplanes $\alpha_{s}=0$. 
The polynomial $\delta=\prod_{s \in \mathcal{S}} \alpha_{s} \in \mathbb{C}[\mathfrak{h}]$ will be important, in part because $\mathfrak{h}^{\text {reg }}=\mathfrak{h} \backslash\{\delta=0\}$ is the subset of $\mathfrak{h}$ on which the action of $W$ is free. Notice that $\delta$ transforms under $W$ by the sign representation sign.

Given a variety $Z$ we will write $D(Z)$ for the ring of differential operators on $Z$. Let $D\left(\mathfrak{h}^{\text {reg }}\right) * W$ denote the skew group ring of $D\left(\mathfrak{h}^{\text {reg }}\right)$ by $W$; thus, by definition, $w f=w(f) w$, for all $f \in D\left(\mathfrak{h}^{\text {reg }}\right)$ and $w \in W$. For $c \in \mathbb{C}$ the rational Cherednik algebra of type $A_{n-1}$ is the $\mathbb{C}$-subalgebra $H_{c}$ of $D\left(\mathfrak{h}^{\text {reg }}\right) * W$ generated by the multiplication operators $\mathfrak{h}^{*} \subseteq \mathbb{C}[\mathfrak{h}]=\mathbb{C}\left[x_{1}, \ldots, x_{n-1}\right]$, the group algebra $\mathbb{C} W$, and the Dunkl operators

$$
y_{i}=\partial_{i}-\sum_{s \in S} c \alpha_{s}\left(y_{i}\right) \alpha_{s}^{-1}(1-s), \quad \text { where } \partial_{i}=\partial / \partial x_{i} .
$$

3.2. By [EG, Theorem 1.3] there is a Poincaré-Birkhoff-Witt isomorphism of $\mathbb{C}$-vector spaces

$$
\mathbb{C}[\mathfrak{h}] \otimes_{\mathbb{C}} \mathbb{C} W \otimes_{\mathbb{C}} \mathbb{C}\left[\mathfrak{h}^{*}\right] \stackrel{\sim}{\rightarrow} H_{c}
$$

under which $\mathbb{C}\left[\mathfrak{h}^{*}\right]$ identifies with the subalgebra of $H_{c}$ generated by $\mathfrak{h}=\bigoplus_{i=1}^{n-1} \mathbb{C} y_{i}$.

Equation 3.2.1 can be interpreted as follows. For any variety $Z$, one has a natural filtration on $D(Z)$ by order of operators and this induces a filtration on $D\left(\mathfrak{h}^{\text {reg }}\right) * W$ and its subalgebras by defining elements of $W$ to have order zero. If $R$ is a subalgebra (or subset) of $D\left(\mathfrak{h}^{\text {reg }}\right) * W$, let $\operatorname{ord}^{n} R$ denote the operators of order $\leq n$ in $R$. The associated graded ring of $R$ will be written $\operatorname{ogr}(R)=\bigoplus \operatorname{ogr}^{n}(R)$, where $\operatorname{ogr}^{n}(R)=$ $\operatorname{ord}^{n}(R) / \operatorname{ord}^{n-1}(R)$ and the resulting graded structure of $\operatorname{ogr}(R)$ will be called the order or ogr gradation.

If we filter $H_{c}$ in this way, then (3.2.1) implies that $\operatorname{ord}^{0} H_{c}=\mathbb{C}[\mathfrak{h}] * W$, $\operatorname{ord}^{1} H_{c}=\mathfrak{h}+\operatorname{ord}^{0} H_{c}$ and $\operatorname{ord}^{i} H_{c}=\left(\operatorname{ord}^{1} H_{c}\right)^{i}$ for $i>1$. Moreover, the associated graded ring ogr $H_{c}=\bigoplus \operatorname{ogr}^{n} H_{c}$ is isomorphic to the skew group ring $\mathbb{C}\left[\mathfrak{h} \oplus \mathfrak{h}^{*}\right] * W$.

3.3. The spherical subalgebra. Let $e \in \mathbb{C} W$ be the trivial idempotent and $e_{-} \in \mathbb{C} W$ be the sign idempotent; thus $e=|W|^{-1} \sum_{w \in W} w$ and $e_{-}=|W|^{-1} \sum_{w \in W} \operatorname{sign}(w) w$. The spherical subalgebra of $H_{c}$ is the algebra $U_{c}=e H_{c} e$ while the related algebra $U_{c}^{-}=e_{-} H_{c} e_{-}$is called the anti-spherical subalgebra. By [BEG2, Proposition 4.1] these algebras are related through the identity

$$
U_{c}=\delta^{-1} U_{c+1}^{-} \delta=e \delta^{-1} H_{c+1} \delta e .
$$

3.4. By (3.3.1), $e H_{c+1} \delta e$ is an $\left(U_{c+1}, U_{c}\right)$-bimodule but the results of this paper require that it is also a progenerator on both sides. This fails for special values of $c \in(-1,0)$ (see [GS, Remarks 3.14]) and so throughout this paper we will need to make the following assumption:

Hypothesis. Set $\mathcal{C}=\left\{z: z=\frac{m}{d}\right.$ where $m, d \in \mathbb{Z}$ with $2 \leq d \leq n$ and $\left.z \notin \mathbb{Z}\right\}$. Assume that $c \in \mathbb{C}$ is such that $c \notin \frac{1}{2}+\mathbb{Z}$. If $c$ is a negative rational number assume further that $c \notin \mathcal{C}$.

Remarks. (1) If $n=2$ and $c \in \frac{1}{2}+\mathbb{N}$, then all the results of this paper do also hold as stated. The reason is that [GS, Theorem 3.3] is easy to prove directly for Cherednik algebras of type $A_{1}$ (see [GS, Remark 3.14(1)]) and this is the only place where the restriction $c \notin \frac{1}{2}+\mathbb{Z}$ is used. 
(2) Using the identities $H_{c} \cong H_{-c}$ and $U_{c} \cong U_{-c}^{-}$from [De, Section 2], analogues of the results in this paper do hold for $c \in \mathcal{C}$. The details are similar to the arguments of [GS, Corollary 3.13] and are left to the interested reader.

3.5. Assume that $c$ satisfies Hypothesis 3.4. Then [GS, Corollary 3.13] implies that the shift functors

$$
S_{c}: U_{c}-\bmod \rightarrow U_{c+1}-\bmod : \quad N \mapsto e H_{c+1} \delta e \otimes_{U_{c}} N
$$

and

$$
\widetilde{S}_{c}: H_{c}-\bmod \rightarrow H_{c+1} \text {-mod }: \quad M \mapsto H_{c+1} \delta e \otimes_{U_{c}} e M
$$

are equivalences of categories. For $i>j \geq 0$ set

$$
B_{j j}(c)=B_{j j}=U_{c+j} \quad \text { and } \quad B_{i j}(c)=B_{i j}=\left(e H_{c+i} \delta e\right)\left(e H_{c+i-1} \delta e\right) \cdots\left(e H_{c+j+1} \delta e\right)
$$

where the multiplication is taken in $D\left(\mathfrak{h}^{\text {reg }}\right) * W$. Since each $e H_{c+i} \delta e$ is projective, multiplication gives a natural isomorphism

$$
e H_{c+i} \delta e \otimes_{U_{c+i-1}} e H_{c+i-1} \delta e \otimes_{U_{c+i-2}} \cdots \otimes_{U_{c+j+1}} e H_{c+j+1} \delta e \cong B_{i j}
$$

As a result we have a Morita $\mathbb{Z}$-algebra

$$
B=B(c)=\bigoplus_{i \geq j \geq 0} B_{i j}
$$

associated to the data $\left\{U_{c+i}, e H_{c+i} \delta e ; i \in \mathbb{N}\right\}$. By setting $E=D\left(\mathfrak{h}^{\text {reg }}\right) * W$ with the order filtration, we see that the requirements $(1-3)$ of $(2.3)$ are fulfilled.

3.6. The Hilbert scheme. We next want to relate $U_{c}$ and $B$ to Hilbert schemes. Let Hilb $\mathbb{C}^{2}$ be the Hilbert scheme of $n$ points on the plane, which we realise as the set of ideals of colength $n$ in the polynomial ring $\mathbb{C}\left[\mathbb{C}^{2}\right]$. If we identify the variety $S^{n} \mathbb{C}^{2}$ of unordered $n$-tuples of points in $\mathbb{C}^{2}$ with the categorical quotient $\mathbb{C}^{2 n} / W$, then the map $\widehat{\tau}: \operatorname{Hilb}^{\mathrm{n}} \mathbb{C}^{2} \longrightarrow S^{n} \mathbb{C}^{2}$ which sends an ideal to its support (counted with multiplicity) is a resolution of singularities [Na, Theorem 1.15].

Consider the $W$-equivariant map $\mathfrak{h} \oplus \mathfrak{h}^{*} \hookrightarrow \mathbb{C}^{2 n}$. To fix notation, let $\mathfrak{h}$ be the hypersurface $\mathbf{z}=0$ in the first copy of $\mathbb{C}^{n}$ and similarly let $\mathfrak{h}^{*}$ be the hypersurface $\mathbf{z}^{*}=0$ in the second copy of $\mathbb{C}^{n}$; thus $\mathbb{C}\left[\mathbb{C}^{2 n}\right]=\mathbb{C}\left[\mathfrak{h} \oplus \mathfrak{h}^{*}\right]\left[\mathbf{z}, \mathbf{z}^{*}\right]$. Define

$$
\operatorname{Hilb}(\mathrm{n})=\widehat{\tau}^{-1}\left(\mathfrak{h} \oplus \mathfrak{h}^{*} / W\right)
$$

In [Ha2, Ha3], Haiman proves a number of fundamental results about $\mathrm{Hilb}^{\mathrm{n}} \mathbb{C}^{2}$. The analogous results for Hilb(n) will be needed in this paper but, by [GS, Lemma 4.9 and Corollary 4.10], they follow routinely from Haiman's work. In particular, $\widehat{\tau}$ restricts to a crepant resolution of singularities $\tau: \operatorname{Hilb}(\mathrm{n}) \rightarrow \mathfrak{h} \oplus \mathfrak{h}^{*} / W$. 
3.7. Write $A^{0}=\mathbb{C}\left[\mathfrak{h} \oplus \mathfrak{h}^{*}\right]^{W}, J^{0}=\mathbb{C}\left[\mathfrak{h} \oplus \mathfrak{h}^{*}\right]$ and set $A^{1}=\mathbb{C}\left[\mathfrak{h} \oplus \mathfrak{h}^{*}\right]^{\text {sign }}$ and $J^{1}=\mathbb{C}\left[\mathfrak{h} \oplus \mathfrak{h}^{*}\right] A^{1}$. For $k>1$, define $A^{k}=\left(A^{1}\right)^{k}$ and $J^{k}=\left(J^{1}\right)^{k}$ for the respective $k^{\text {th }}$ powers using multiplication in $\mathbb{C}\left[\mathfrak{h} \oplus \mathfrak{h}^{*}\right]$. Finally, write

$$
A=\bigoplus_{k \geq 0} A^{k} \delta^{k} \cong A^{0}\left[A^{1} \delta t\right] \quad \text { and } \quad S=\bigoplus_{k \geq 0} J^{k} \delta^{k} \cong \mathbb{C}\left[\mathfrak{h} \oplus \mathfrak{h}^{*}\right]\left[J^{1} \delta t\right]
$$

for the corresponding Rees rings at the ideals $A^{1} \delta$, respectively $J^{1} \delta$. By [GS, Corollary 4.10], Hilb(n) $=$ $\operatorname{Proj}(A)$. Moreover $X_{n}=\operatorname{Proj}(S)$ is the reduced fibre product

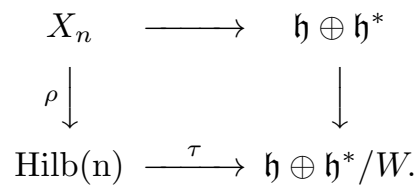

and the map $\rho$ is flat of degree $n$ !.

Associated to these objects we have three important vector bundles: the rank $n$ tautological bundle $\mathcal{B}$ on $\operatorname{Hilb}(\mathrm{n})$ whose fibre above $I \in \operatorname{Hilb}(\mathrm{n})$ is the $n$-dimensional vector space $\mathbb{C}\left[\mathbb{C}^{2}\right] / I$; the Procesi bundle $\mathcal{P}=\rho_{*} \mathcal{O}_{X_{n}}$ of rank $n$ !; and the line bundle $\mathcal{L}=\wedge^{n} \mathcal{B}$. By [Ha1, Proposition 2.12] $\mathcal{L} \cong \mathcal{O}_{\text {Hilb(n) }}(1)$, the ample line bundle associated to the isomorphism $\operatorname{Hilb}(\mathrm{n})=\operatorname{Proj}(A)$.

3.8. The given definitions of $A$ and $S$ agree with those used in Section 6 of [GS], but not with those used in the earlier sections of that paper where the (isomorphic) rings $A^{0}\left[A^{1} t\right]$ and $\mathbb{C}\left[\mathfrak{h} \oplus \mathfrak{h}^{*}\right]\left[J^{1} t\right]$ are used. The reason for caring about the distinction is that $W$ acts on $S$ via its natural action on $\mathbb{C}\left[\mathfrak{h} \oplus \mathfrak{h}^{*}\right]$, and on $\mathcal{P}=\rho_{*} \mathcal{O}_{X_{n}}$ by the permutation action on $X_{n}$. By [GS, (4.6) and Corollary 4.10] the induced actions agree under our chosen action:

$$
\mathrm{H}^{0}\left(\mathrm{Hilb}(\mathrm{n}), \mathcal{P} \otimes \mathcal{L}^{d}\right) \cong J^{d} \delta^{d} \quad \text { as } W-\text { representations. }
$$

In contrast, $W$ acts on $\delta$ with the sign representation and so acts on $J^{d}$ by $\operatorname{sign}^{\otimes d}$ [GS, loc.cit.].

3.9. Let $\widehat{A}=\bigoplus_{i \geq j \geq 0} A^{i-j} \delta^{i-j}$ be the $\mathbb{Z}$-algebra associated to $A$ as in (2.2). By [GS, Theorem 6.4(2)]

$$
\operatorname{ogr} B=e \widehat{A} e \cong \widehat{A}
$$

whenever $c$ satisfies Hypothesis 3.4. Hence requirement (4) of (2.3) is also satisfied. Combined with (3.5) this implies that:

If $c \in \mathbb{C}$ satisfies Hypothesis 3.4, then Theorem 1.2 holds for $U_{c}$ and, moreover, we can apply all the results from Section 2. 
3.10. Partitions. We will use the same conventions for partitions and $W$-representations as was used in [GS]. Thus Irrep $(W)$ denotes the set of irreducible representations of $W$, up to isomorphism. These irreducible representations will be parametrised by partitions $\mu=\left(\mu_{1} \geq \mu_{2} \geq \cdots \geq \mu_{\ell}>0\right)$ of $n$, where the understanding is that $\mu_{i}=0$ for $i>\ell$. The Ferrers diagram of $\mu$ is the set of lattice points

$$
d(\mu)=\left\{(i, j) \in \mathbb{N} \times \mathbb{N}: j<\mu_{i+1}\right\} .
$$

Following the French style, the diagram is drawn with the $i$-axis vertical and the $j$-axis horizontal, so the parts of $\mu$ are the lengths of the rows, and $(0,0)$ is the lower left corner. The arm $a(x)$ and the $\operatorname{leg} l(x)$ of a point $x \in d(\mu)$ denote the number of points strictly to the right of $x$ and above $x$, respectively. See [GS, (2.6.1)] for a typical example. The transpose partition $\mu^{t}$ is obtained from $\mu$ by reflecting the Ferrers diagram about the line $y=x$, in other words exchanging the rows and columns of $\mu$.

The partition statistic of a partition $\mu$ is $n(\mu)=\sum_{i} \mu_{i}(i-1)$. We will always use the dominance ordering of partitions as in, for example [Mac, p.7]; thus if $\lambda$ and $\mu$ are partitions of $n$ then $\lambda \geq \mu$ if and only if $\sum_{i=1}^{k} \lambda_{i} \geq \sum_{i=1}^{k} \mu_{i}$ for all $k \geq 1$. Thus, as in [Mac, Example 1, p.116], the trivial representation triv is labelled by $(n)$ while the sign representation sign is parametrised by $\left(1^{n}\right)$ and so triv $>$ sign.

3.11. For a pair of partitions $\mu, \lambda$ let $K_{\mu \lambda}(t, s)$ be the Kostka-Macdonald coefficients defined in [Mac, VI, (8.11)]. Their specialisations yield the classical Kostka numbers $K_{\mu \lambda}=K_{\mu \lambda}(0,1)$. By definition, these numbers give the transition matrix between the bases of the ring of symmetric functions given by the Schur functions, $s_{\mu}$, and by the monomial symmetric functions, $m_{\mu}$; thus $s_{\mu}=\sum_{\lambda \vdash n} K_{\mu \lambda} m_{\lambda}$. By [Mac, I, (6.5)], $K_{\mu \mu}=1$ and $K_{\mu \lambda}=0$ unless $\mu \geq \lambda$.

3.12. The punctual Hilbert scheme. Let $\mathbf{0} \in S^{n} \mathbb{C}^{2}$ be the zero orbit. The punctual Hilbert scheme, $\mathrm{Z}_{\mathrm{n}}$, is defined to be $\widehat{\tau}^{-1}(\mathbf{0})$, the fibre of the resolution $\widehat{\tau}: \operatorname{Hilb}^{\mathrm{n}} \mathbb{C}^{2} \rightarrow S^{n} \mathbb{C}^{2}$ above 0. By [Ha1, Proposition 2.10] $\mathrm{Z}_{\mathrm{n}}$ is reduced. Since $\mathbf{0} \in \mathfrak{h} \oplus \mathfrak{h}^{*} / W$ we also have $\mathrm{Z}_{\mathrm{n}}=\tau^{-1}(\mathbf{0})$. Let $\mathfrak{m}=\mathbb{C}\left[\mathfrak{h} \oplus \mathfrak{h}^{*}\right]_{+}^{W} \triangleleft \mathbb{C}\left[\mathfrak{h} \oplus \mathfrak{h}^{*}\right]^{W}$ be the maximal ideal corresponding to $\mathbf{0}$. Since $\operatorname{Hilb}(\mathrm{n})=\operatorname{Proj} A$, we have $\mathrm{Z}_{\mathrm{n}}=\operatorname{Proj}(A / A \mathfrak{m})$.

Lemma. Let $\mathcal{P}$ and $\mathcal{L}$ be the bundles defined in (3.7). Then, for all $k \geq 0$, there is a vector space isomorphism $\mathrm{H}^{0}\left(\mathrm{Z}_{\mathrm{n}}, \mathcal{L}^{k}\right) \cong A^{k} \delta^{k} / A^{k} \delta^{k} \mathfrak{m}$ and a $W$-equivariant isomorphism of vector spaces

$$
\mathrm{H}^{0}\left(\mathrm{Z}_{\mathrm{n}}, \mathcal{P} \otimes \mathcal{L}^{k}\right) \cong J^{k} \delta^{k} / J^{k} \delta^{k} \mathfrak{m} .
$$

Proof. Once we set up the notation, this is an easy consequence of [Ha3, Theorem 2.2]. We first prove (3.12.1). Let $\mathcal{P}_{1}, \mathcal{B}_{1}$ and $\mathcal{L}_{1}=\bigwedge^{n} \mathcal{B}_{1}$ denote the vector bundles on Hilb $\mathbb{C}^{2}$ analogous to $\mathcal{P}, \mathcal{B}$ and $\mathcal{L}$ on $\operatorname{Hilb}(\mathrm{n})$, as in [GS, (4.5)]. By construction, the restriction of $\mathcal{P}_{1}$ (respectively $\mathcal{L}_{1}$ ) to $\mathrm{Z}_{\mathrm{n}}$ equals the restriction of $\mathcal{P}$ (respectively $\mathcal{L}$ ). Set $\ell=k n$ and define $R(n, \ell)=\mathrm{H}^{0}\left(\operatorname{Hilb}^{\mathrm{n}} \mathbb{C}^{2}, \mathcal{P}_{1} \otimes \mathcal{B}_{1}^{\ell}\right)$, where $\mathcal{B}_{1}^{\ell}=\mathcal{B}_{1}^{\otimes \ell}$. Finally, let $W^{k} \subset \mathfrak{S}_{\ell}$ act on $\mathcal{B}_{1}^{\ell}$ by permutations.

Let $\mathfrak{m}_{1} \triangleleft \mathbb{C}\left[\mathbb{C}^{2 n}\right]$ be the maximal ideal corresponding to $\mathbf{0}$. It follows from [Ha3, Theorem 2.2] that $R(n, \ell) / R(n, \ell) \mathfrak{m}_{1}=\mathrm{H}^{0}\left(\mathrm{Z}_{\mathrm{n}}, \mathcal{P}_{1} \otimes \mathcal{B}_{1}^{\ell}\right)$ while, by definition, if $\epsilon_{k}$ denotes the sign representation of $W^{k}$ then 
$\mathcal{L}_{1}^{k}=\left(\mathcal{B}_{1}^{\ell}\right)^{\epsilon_{k}}$. As the action of $W^{k}$ is trivial on $\mathcal{P}_{1}$, we therefore obtain

$$
\mathrm{H}^{0}\left(\mathrm{Z}_{\mathrm{n}}, \mathcal{P} \otimes \mathcal{L}^{k}\right)=\mathrm{H}^{0}\left(\mathrm{Z}_{\mathrm{n}}, \mathcal{P}_{1} \otimes \mathcal{L}_{1}^{k}\right)=\mathrm{H}^{0}\left(\mathrm{Z}_{\mathrm{n}},\left(\mathcal{P}_{1} \otimes \mathcal{B}_{1}^{\ell}\right)^{\epsilon_{k}}\right)=\mathrm{H}^{0}\left(\mathrm{Z}_{\mathrm{n}}, \mathcal{P}_{1} \otimes \mathcal{B}_{1}^{\ell}\right)^{\epsilon_{k}}=R(n, \ell)^{\epsilon_{k}} / R(n, \ell)^{\epsilon_{k}} \mathfrak{m}_{1} .
$$

By [GS, Lemma 4.9(1) and (4.6.2)] $R(n, \ell)^{\epsilon_{k}} / R(n, \ell)^{\epsilon_{k}} \mathfrak{m}_{\perp} \cong J^{k} \delta^{k} / J^{k} \delta^{k} \mathfrak{m} W$-equivariantly. Combined with the last display, this proves (3.12.1).

By [GS, Lemmas 4.4(1) and 4.9(1)], $A^{k} \delta^{k}$ equals the triv-isotypic component of $J^{k} \delta^{k}$, while $\mathcal{O}_{\text {Hilb(n) }}=\mathcal{P}^{W}$. Substituting these observations into (3.12.1) gives

$$
\mathrm{H}^{0}\left(\mathrm{Z}_{\mathrm{n}}, \mathcal{L}^{k}\right) \cong \mathrm{H}^{0}\left(\mathrm{Z}_{\mathrm{n}},\left(\mathcal{P} \otimes \mathcal{L}^{k}\right)\right)^{W} \cong A^{k} \delta^{k} / A^{k} \delta^{k} \mathfrak{m}
$$

as required.

3.13. Torus action. In Section 5 we will need two refinements on Lemma 3.12: first that the isomorphisms are bigraded under the appropriate torus action and second that we can determine the dimensions of $\mathrm{H}^{0}\left(\mathrm{Z}_{\mathrm{n}}, \mathcal{L}^{k}\right)$ and $\mathrm{H}^{0}\left(\mathrm{Z}_{\mathrm{n}}, \mathcal{P} \otimes \mathcal{L}^{k}\right)$. Both results are implicit in Haiman's papers, but take a while to explain.

We begin with the torus action. The torus $\mathbb{T}^{2}=\left(\mathbb{C}^{*}\right)^{2}$ acts linearly on $\mathbb{C}^{2}$ as the group of diagonal matrices of the form $\tau_{s, t}=\operatorname{diag}\left\{s^{-1}, t^{-1}\right\}$; thus, as in $[\mathrm{Ha} 3,(12)], \mathbb{T}^{2}$ acts on $\mathbb{C}\left[\mathbb{C}^{2}\right]=\mathbb{C}[x, y]$ by $\tau_{s, t} x=s x, \tau_{s, t} y=t y$. This induces a $\mathbb{T}^{2}$-action on $S^{n} \mathbb{C}^{2}$ and Hilb $\mathbb{C}^{2}$; furthermore, since $\mathfrak{h} \oplus \mathfrak{h}^{*} / W \subset S^{n} \mathbb{C}^{2}$ is $\mathbb{T}^{2}$-stable, there is an induced $\mathbb{T}^{2}$-action on Hilb(n). This action further restricts to the punctual Hilbert scheme $Z_{n}$ since the zero orbit in $\mathfrak{h} \oplus \mathfrak{h}^{*} / W$ is invariant under $\mathbb{T}^{2}$.

As in [Ha3, Section 2, p.377], there is an induced $\mathbb{T}^{2}$-equivariant structure on the tautological bundle $\mathcal{B}$ and on the Procesi bundle $\mathcal{P}$ arising from the action of $\mathbb{T}^{2}$ on $\mathbb{C}\left[\mathbb{C}^{2}\right]$, respectively $\mathbb{C}\left[\mathfrak{h} \oplus \mathfrak{h}^{*}\right]$. Consequently, $\mathcal{L}=\wedge^{n} \mathcal{B}$ is also a $\mathbb{T}^{2}$-equivariant sheaf. Analogously, the vector bundles $\mathcal{B}_{1}, \mathcal{P}_{1}, \mathcal{L}_{1}$ over Hilb $\mathbb{C}^{2}$ introduced in the proof of Lemma 3.12 have natural $\mathbb{T}^{2}$-equivariant structures.

Of course, one also obtains induced actions of $\mathbb{T}^{2}$ on the sections of each of these bundles. This action can be equivalently described by a $\mathbb{Z}^{2}$-grading: an element $f$ is homogeneous of weight $(i, j)$ if $\tau_{s, t} f=s^{i} t^{j} f$. If $M=\bigoplus_{i, j} M^{i j}$ is a bigraded decomposition of a module $M$ arising from such a $\mathbb{T}^{2}$-action, then the Poincaré series of $M$ is the Laurent series $p(M, s, t)=\sum_{i, j} s^{i} t^{j} \operatorname{dim} M^{i j}$. The reader should note that the variables $s$ and $t$ appear in the opposite order in [Ha3, (46)].

3.14. The action of $\mathbb{T}^{2}$ on $\mathbb{C}\left[\mathfrak{h} \oplus \mathfrak{h}^{*}\right]$ also induces a bigrading on $A^{k}$ and $J^{k}$ and the element $\delta$ is bihomogeneous for this action with weight $(N, 0)$, where $N=n(n-1) / 2$. We have the following refinement of Lemma 3.12.

Corollary. The isomorphisms of Lemma 3.12 restrict to identifications of bigraded components

$$
\mathrm{H}^{0}\left(\mathrm{Z}_{\mathrm{n}}, \mathcal{L}^{k}\right)^{i j} \cong\left(\frac{A^{k} \delta^{k}}{A^{k} \delta^{k} \mathfrak{m}}\right)^{i+N k, j} \quad \text { and } \quad \mathrm{H}^{0}\left(\mathrm{Z}_{\mathrm{n}}, \mathcal{P} \otimes \mathcal{L}^{k}\right)^{i j} \cong\left(\frac{J^{k} \delta^{k}}{J^{k} \delta^{k} \mathfrak{m}}\right)^{i+N k, j} .
$$

Proof. Set $\ell=n k$, for some $k$, and let $\mathbb{J}^{k}$ denote the $\mathbb{C}\left[\mathbb{C}^{2 n}\right]$ analogue of $J^{k}$, as in [GS, (4.3)] . By [GS, (4.6.1)], there is an isomorphism

$$
\mathbb{J}^{k} \cong R(n, \ell)^{\epsilon_{k}} \cong \mathrm{H}^{0}\left(\operatorname{Hilb}^{\mathrm{n}} \mathbb{C}^{2}, \mathcal{P}_{1} \otimes \mathcal{L}_{1}^{k}\right)
$$


We claim that (3.14.1) is $\mathbb{T}^{2}$-equivariant. For the second isomorphism this is just [Ha3, (69)]. Note, here, that the extra variables $a_{i}$ and $b_{j}$ used in the definition of $R(n, \ell)$ in $[\mathrm{Ha} 3,(17)]$ have bidegree $(1,0)$, respectively $(0,1)$. The first isomorphism in (3.14.1) is constructed explicitly in the third paragraph of the proof of [Ha2, Proposition 4.11.1] and is clearly $\mathbb{T}^{2}$-equivariant, as claimed.

As in the proof of Lemma 3.12, $J^{k} / J^{k} \mathfrak{m}=\mathbb{J}^{k} / \mathbb{J}^{k} \mathfrak{m}_{1} \cong \mathrm{H}^{0}\left(\mathrm{Z}_{\mathrm{n}}, \mathcal{P} \otimes \mathcal{L}^{k}\right)$ and, by (3.14.1), this isomorphism is $\mathbb{T}^{2}$-equivariant. Since $\delta$ is bihomogeneous of degree $(N, 0)$, this proves the second isomorphism of the lemma. As in the proof of Lemma 3.12, the first equation follows by taking $W$-fixed points.

3.15. For the next result we need some notation. By [Ha3, Proposition 3.1] the $\mathbb{T}^{2}$-fixed points on Hilb(n) are labelled by partitions of $n$ : to any partition $\eta$ we associate the monomial ideal

$$
I_{\eta}=\mathbb{C} \cdot\left\{x^{r} y^{s}:(r, s) \notin d(\eta)\right\} \unlhd \mathbb{C}[x, y]
$$

regarded as a point of $\operatorname{Hilb}(\mathrm{n})$. We also recall that the Catalan number $C_{n}^{(k)}$ is defined to be

$$
C_{n}^{(k)}=\frac{1}{k n+1}\left(\begin{array}{c}
(k+1) n \\
n
\end{array}\right)
$$

Lemma. For all $k \geq 1$, we have $\operatorname{dim} \mathrm{H}^{0}\left(\mathrm{Z}_{\mathrm{n}}, \mathcal{P} \otimes \mathcal{L}^{k-1}\right)=(k n+1)^{n-1}$ and $\operatorname{dim} \mathrm{H}^{0}\left(\mathrm{Z}_{\mathrm{n}}, \mathcal{L}^{k}\right)=C_{n}^{(k)}$.

Proof. As in the proof of Lemma 3.12, we may replace $\mathcal{P}$ and $\mathcal{L}$ by $\mathcal{P}_{1}$ and $\mathcal{L}_{1}$ and so, by [Ha3, Theorem 2.2], the hypothesis of [Ha1, Theorem 2] is now valid. Thus, by [Ha1, (1.7) and Theorem 2], and in the notation of that paper, $\operatorname{dim} \mathrm{H}^{0}\left(\mathrm{Z}_{\mathrm{n}}, \mathcal{L}_{1}^{k}\right)=C_{n}^{(k)}(1,1)=C_{n}^{(k)}$. This proves the second formula.

For a partition $\mu$ of $n$, set $\Omega(\mu)=\prod_{x \in d(\mu)}\left(1-s^{1+l(x)} t^{-a(x)}\right)\left(1-s^{-l(x)} t^{1+a(x)}\right)$, and write

$$
B_{\mu}=\sum_{(x, y) \in d(\mu)} s^{x} t^{y}, \quad \Pi_{\mu}=\prod_{\substack{(x, y) \in d(\mu) \\(x, y) \neq(0,0)}}\left(1-s^{x} t^{y}\right) \quad \text { and } \quad P_{\mu}=\sum_{\lambda} s^{n(\mu)} K_{\lambda \mu}\left(t, s^{-1}\right) \operatorname{dim} \lambda .
$$

We claim that $\mathrm{H}^{0}\left(\mathrm{Z}_{\mathrm{n}}, \mathcal{P}_{1} \otimes \mathcal{L}_{1}^{k-1}\right)$ has the Poincaré series

$$
p\left(\mathrm{H}^{0}\left(\mathrm{Z}_{\mathrm{n}}, \mathcal{P}_{1} \otimes \mathcal{L}_{1}^{k-1}\right), s, t\right)=\sum_{\mu} P_{\mu} s^{(k-1) n(\mu)} t^{(k-1) n\left(\mu^{t}\right)}(1-s)(1-t) \Pi_{\mu} B_{\mu} \Omega(\mu)^{-1} .
$$

We prove the claim by following the derivation of [Ha3, Theorem 3.2]. A locally free, $\mathbb{T}^{2}$-equivariant resolution

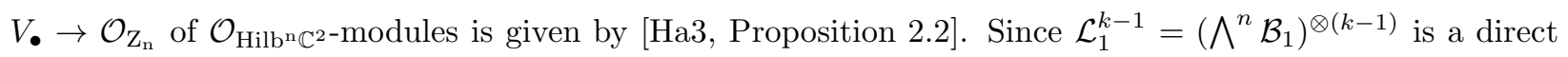
summand of $\mathcal{B}_{1}^{\otimes n(k-1)}$, [Ha3, Theorem 2.2] shows that $\mathrm{H}^{i}\left(\mathrm{Hilb}^{\mathrm{n}} \mathbb{C}^{2}, \mathcal{P}_{1} \otimes \mathcal{L}_{1}^{k-1} \otimes \mathcal{O}_{\mathrm{Z}_{\mathrm{n}}}\right)=\mathrm{H}^{i}\left(\mathrm{Z}_{\mathrm{n}}, \mathcal{P}_{1} \otimes \mathcal{L}_{1}^{k-1}\right)=0$ for all $i>0$. Combining these observations means that

$$
\begin{aligned}
p\left(\mathrm{H}^{0}\left(\mathrm{Z}_{\mathrm{n}}, \mathcal{P}_{1} \otimes \mathcal{L}_{1}^{k-1}\right), s, t\right) & =p\left(\mathrm{H}^{0}\left(\operatorname{Hilb}^{\mathrm{n}} \mathbb{C}^{2}, \mathcal{P}_{1} \otimes \mathcal{L}_{1}^{k-1} \otimes \mathcal{O}_{\mathrm{Z}_{\mathrm{n}}}\right), s, t\right) \\
& =\sum_{j \geq 0}(-1)^{j} p\left(\mathrm{H}^{0}\left(\operatorname{Hilb}^{\mathrm{n}} \mathbb{C}^{2}, \mathcal{P}_{1} \otimes \mathcal{L}_{1}^{k-1} \otimes V_{j}\right), s, t\right)
\end{aligned}
$$


By [Ha3, (35)], the sheaves $\mathcal{P}_{1} \otimes \mathcal{L}_{1}^{k-1} \otimes V_{j}$ are acyclic for the global section functor. Thus, combining the last displayed equation with the Atiyah-Bott-Lefschetz formula [Ha3, Proposition 3.2] gives

$$
\begin{aligned}
p\left(\mathrm{H}^{0}\left(\mathrm{Z}_{\mathrm{n}}, \mathcal{P}_{1} \otimes \mathcal{L}_{1}^{k-1}\right), s, t\right) & =\sum_{\mu} \sum_{j \geq 0}(-1)^{j} p\left(\mathcal{P}_{1}\left(I_{\mu}\right) \otimes \mathcal{L}_{1}^{k-1}\left(I_{\mu}\right) \otimes V_{j}\left(I_{\mu}\right), s, t\right) \Omega(\mu)^{-1} \\
& =\sum_{\mu} p\left(\mathcal{L}_{1}\left(I_{\mu}\right), s, t\right)^{k-1} \Omega(\mu)^{-1} \sum_{j \geq 0}(-1)^{j} p\left(\mathcal{P}_{1}\left(I_{\mu}\right) \otimes V_{j}\left(I_{\mu}\right), s, t\right) .
\end{aligned}
$$

As was shown in the proof of [GS, Proposition 4.8], $p\left(\mathcal{L}_{1}\left(I_{\mu}\right), s, t\right)=s^{n(\mu)} t^{n\left(\mu^{t}\right)}$, while the final sum in (3.15.3) is computed in [Ha3, (89)] (the individual terms in [Ha3, (89)] are defined in Equations (71), (73) and (88) of [Ha3]; note, however, that the convention [Ha3, (46)] for Poincaré series differs from ours). These computations combine to give (3.15.2), as claimed.

By [Ha1, (1.9)] we have $\sum_{x \in d(\mu)} l(x)=n(\mu)$ and, similarly, $\sum_{x \in d(\mu)} a(x)=n\left(\mu^{t}\right)$. Applying these formulæ to (3.15.2) gives

$$
p\left(\mathrm{H}^{0}\left(\mathrm{Z}_{\mathrm{n}}, \mathcal{P}_{1} \otimes \mathcal{L}_{1}^{k-1}\right), s, t\right)=\sum_{\mu} \frac{P_{\mu}(1-s)(1-t) s^{k n(\mu)} t^{k n\left(\mu^{t}\right)} \Pi_{\mu} B_{\mu}}{\prod_{x \in d(\mu)}\left(t^{a(x)}-s^{1+l(x)}\right)\left(s^{l(x)}-t^{1+a(x)}\right)} .
$$

The right hand side of this equation is the same as [GH1, (3.27)], provided one specialises the formal variable $X=\left(x_{1}, x_{2}, \ldots\right)$ from [GH1, (3.27)] to $x_{1}=\cdots=x_{n}=1$ and $x_{i}=0$ for $i>n$. Now [GH1, (3.27)] can be computed from [GH1, Theorem 4.1]. Let $e_{n}$ denote the $n^{\text {th }}$ elementary symmetric function and note that, if $q=1$ and $X$ is as above, then $e_{n}\left[X\left(1+q+\ldots+q^{k n}\right)\right]=e_{n}[X(k n+1)]=(k n+1)^{n}$ in the notation of [GH1, Introduction]. Therefore, specialising [GH1, Theorem 4.1] to $q=1$ (which means taking $s=t=1$ in our notation) gives

$$
\operatorname{dim} \mathrm{H}^{0}\left(\mathrm{Z}_{\mathrm{n}}, \mathcal{P}_{1} \otimes \mathcal{L}_{1}^{k-1}\right)=\frac{1}{k n+1} e_{n}\left[X\left(1+q+\ldots+q^{k n}\right)\right]=(k n+1)^{n-1},
$$

as required.

3.16. We note for use in (5.12) that the derivation of (3.15.2) also yields

$$
p\left(\mathrm{H}^{0}\left(\mathrm{Z}_{\mathrm{n}}, \mathcal{L}^{k}\right), s, t\right)=\sum_{\mu} s^{k n(\mu)} t^{k n\left(\mu^{t}\right)}(1-s)(1-t) \Pi_{\mu} B_{\mu} \Omega(\mu)^{-1} .
$$

This is the two variable Catalan-like number $C_{n}^{(k)}(s, t)$ of Garsia and Haiman, as defined in [Ha1, (1.10)].

\section{Representations of $H_{c}$ And coherent sheaves on Hilb(n)}

4.1. Throughout the section we assume that $c \in \mathbb{C}$ satisfies Hypothesis 3.4 although, by the observations from Remark 3.4(2), analogues of the results proved here do hold for more general values of $c$.

The results of the previous sections allow us to associate a coherent sheaf $\Phi_{\Lambda}(M)$ on $\operatorname{Hilb}(\mathrm{n})$ to any $U_{c^{-}}$ or $H_{c}$-module $M$ with a good filtration $\Lambda$ and in this section we investigate the general consequences of this construction. These results will begin to explain one of the central theses of this paper: $\Phi_{\Lambda}(M)$ carries much deeper information about the structure of $M$ than does, for example, the associated graded $A^{0}$-module 
$\operatorname{gr}_{\Lambda} M$. One reason for this is that the fundamental Morita equivalence between $U_{c}$ and $U_{c+1}$, and with it the more subtle information about $U_{c}$-modules, is lost in passing to the associated graded rings but survives in our approach - in the notation of $(3.7)$ it becomes the shift functor $(\mathcal{L} \otimes-$ ) for coherent sheaves (see Lemma 4.4). In Proposition 4.11, we show that there are intriguing connections between the map $\Phi$ and the Bridgeland-King-Reid equivalences.

4.2. Associated sheaves. Given a filtered noetherian $\operatorname{ring} R$, write $R$-Filt for the category of $R$-modules equipped with increasing exhaustive filtrations and whose morphisms respect those filtrations. Let $R$-filt be the full subcategory of $R$-Filt consisting of modules with good filtrations. By (2.2) and (3.9.1), graded modules over $\operatorname{ogr} B \cong \widehat{A}$ are the same as those over $A$. In the notation of (2.4) we therefore have a functor

$$
\Phi: U_{c^{-}} \text {Filt } \longrightarrow \text { Qcoh Hilb(n) } \quad(M, \Lambda) \mapsto \Phi_{\Lambda}(M)=\pi\left(\operatorname{gr}_{\Lambda} \widetilde{M}\right)
$$

where $\pi$ is the quotient map from $A$-Grmod to $A$-Qgr $\simeq$ Qcoh Hilb(n). Lemma 2.5(1) implies that $\Phi$ restricts to a functor $\Phi: U_{c}$-filt $\rightarrow$ Coh Hilb(n).

We will often abuse notation by writing just $\Phi(M)$ when the filtration on $M$ is clearly understood, but we will write $\Phi_{\Lambda}(M)=\Phi_{\Lambda}^{c}(M)$ if we need to specify the initial algebra $U_{c}$.

Similarly, we have a functor $\widehat{\Phi}: H_{c}$-Filt $\rightarrow$ Qcoh Hilb(n). More precisely, if $(N, \Gamma) \in H_{c^{-}}$Filt then applying the functor $E_{c}=\left(e H_{c} \otimes-\right)$ to $(N, \Gamma)$ gives an object $\left(e N, \Gamma_{E}\right) \in U_{c}$-Filt by setting $\Gamma_{E}^{k}(e N)=e \Gamma^{k}(N)$. Thus we can extend $\Phi$ to

$$
\widehat{\Phi}: H_{c} \text {-Filt } \longrightarrow \text { Qcoh Hilb(n) } \quad(N, \Gamma) \mapsto \Phi_{\Lambda}(M) \quad \text { for }(M, \Lambda)=\left(e N, \Gamma_{E}\right) .
$$

If $(N, \Gamma) \in H_{c^{-}}$filt, then $\operatorname{gr}_{\Lambda} e N=\bigoplus e \Gamma^{n} N / e \Gamma^{n-1} N$ is a homomorphic image of the finitely generated $\mathbb{C}\left[\mathfrak{h} \oplus \mathfrak{h}^{*}\right]^{W}$-module $\operatorname{gr}_{\Gamma} N$ and so $\Lambda$ is also a good filtration. Thus, as before, it follows from Lemma 2.5 that $\widehat{\Phi}$ restricts to a functor $\widehat{\Phi}: H_{c}$-filt $\rightarrow$ Coh Hilb(n) .

4.3. Warning. The careful reader has noticed that we have two filtrations in play at the moment. In (3.9.1) we used the order filtration on the bimodules $B_{i j}$, whilst in (2.4.1) and (4.2) we have insisted on the tensor product filtration for modules. For general modules these two filtrations can differ (an example is given in [GS, (7.4)]) and so one can ask whether $\Phi\left(U_{c}\right)$ actually equals $\mathcal{O}_{\text {Hilb(n) }}$. It does, by [GS, Lemma 7.2].

This potential ambiguity occurs several times in this paper — one instance occurs in the next lemma-but in each case it is resolved by [GS, Lemma 7.2].

4.4. Shift Functors. Recall from (3.7) that $\mathcal{L} \cong \mathcal{O}_{\mathrm{Hilb}(\mathrm{n})}(1)$, the Serre twisting sheaf corresponding to the Rees ring $A$. The role of the shift functor $(\mathcal{L} \otimes-)$ in Qcoh Hilb(n) is played by the shift functor $S_{c}$ in the noncommutative world:

Lemma. Assume that $c \in \mathbb{C}$ satisfies Hypothesis 3.4 and let $(M, \Lambda) \in U_{c}$-Filt. Define $\left(M^{\prime}, \Lambda^{\prime}\right) \in U_{c+1}$-Filt by setting $M^{\prime}=S_{c}(M)=\left(e H_{c+1} \delta e\right) \otimes_{U_{c}} M$ together with the tensor product filtration $\Lambda^{\prime}$ from by (2.4.1). Then $\Phi_{\Lambda^{\prime}}^{c+1}\left(M^{\prime}\right) \cong \Phi_{\Lambda}^{c}(M) \otimes \mathcal{L}$. 
Proof. Recall from (3.5.1) the definition of $B_{i j}(c)$. By construction $M(i)=B_{i 0}(c) \otimes_{U_{c}} M$ and

$$
M^{\prime}(i)=B_{i 0}(c+1) \otimes_{U_{c+1}} M^{\prime}=B_{i+1,0}(c) \otimes_{U_{c}} M=M(i+1) .
$$

As in (4.3) we should be careful to compare the two filtrations on $M^{\prime}(i)$ given by this equality. By definition and [GS, Lemma 7.2], $\Lambda^{\prime}$ is given by

$$
\begin{aligned}
&\left(\Lambda^{\prime}\right)^{k} M^{\prime}(i)=\sum_{u+v+w=k} \operatorname{ord}^{u} B_{i 0}(c+1) \otimes \operatorname{ord}^{v} B_{10}(c) \otimes \Lambda^{w} M \\
&=\sum_{x+w=k} \operatorname{ord}^{x} B_{i+1,0}(c) \otimes \Lambda^{w} M=\Lambda^{k} M(i+1) .
\end{aligned}
$$

Thus (4.4.1) is also an equality of filtered modules.

The sheaf $\Phi_{\Lambda}(M)$ on Hilb(n) corresponds to the graded $A$-module $\operatorname{gr}_{\Lambda} \widetilde{M}=\bigoplus_{i \geq 0} \operatorname{gr}_{\Lambda} M(i)$. The action of $A^{j}$ taking $\operatorname{gr}_{\Lambda} M(i+1)$ to $\operatorname{gr}_{\Lambda} M(i+j+1)$ is induced from the mapping

$$
B_{i+j+1, i+1}(c) \otimes_{U_{c+i+1}} M(i+1) \longrightarrow M(i+j+1) .
$$

Similarly the action of $A^{j}$ on $\operatorname{gr}_{\Lambda^{\prime}} M^{\prime}(i)$ is induced from the map $B_{i+j, i}(c+1) \otimes_{U_{c+i+1}} M^{\prime}(i) \rightarrow M^{\prime}(i+j)$. Since $B_{i+j+1, i+1}(c)=B_{i+j, i}(c+1)$, it follows from (4.4.1) that these actions are equal for all $j$ and all $i \geq 0$. Thus, (4.4.2) implies that $\left(\operatorname{gr}_{\Lambda} \widetilde{M}\right)[1]=\bigoplus_{i \geq 0} \operatorname{gr}_{\Lambda} M(i+1)$ and $\operatorname{gr}_{\Lambda^{\prime}} \widetilde{M^{\prime}}=\bigoplus_{i \geq 0} \operatorname{gr}_{\Lambda^{\prime}} M^{\prime}(i)$ are equal as graded $A$-modules. Since $\mathcal{L} \cong \mathcal{O}_{\mathrm{Hilb}(\mathrm{n})}(1)$, this is equivalent to the conclusion of the lemma.

4.5. Many of the results of this paper can be interpreted as saying that important $H_{c}$-modules correspond via $\widehat{\Phi}$ to important sheaves on Hilb(n). We give one example of this philosophy here - for the module $H_{c}$ itself. Comparing this result with (3.7.2) shows that one can think of $H_{c}$ as a noncommutative analogue of the isospectral Hilbert scheme $X_{n}$.

Theorem. Assume that $c \in \mathbb{C}$ satisfies Hypothesis 3.4. Then ogr $\left(\widetilde{e H_{c}}\right)=\bigoplus_{k \geq 0} e J^{k} \delta^{k}$ and $\widehat{\Phi}_{\text {ord }}\left(H_{c}\right) \cong \mathcal{P}$, the Procesi bundle on $\operatorname{Hilb}(\mathrm{n})$.

Proof. We emphasise that if we begin with $(N, \Lambda)=\left(H_{c}\right.$, ord $)$, then $\widehat{\Phi}\left(H_{c}\right)$ is defined by the tensor product filtrations on the summands $B_{k 0} \otimes_{U_{c}} e H_{c}$ of $\widetilde{e H_{c}}=\bigoplus B_{k 0} \otimes_{U_{c}} e H_{c} \cong \bigoplus N(k)$ rather than the ord filtration. However, by [GS, Lemma 7.2], these filtrations are equal. So the identity $\operatorname{ogr}\left(\widetilde{e H_{c}}\right)=\bigoplus e J^{k} \delta^{k}$ of graded vector spaces is immediate from [GS, Proposition 6.5]. That this is an identity of $A$-modules then follows from [GS, Lemma 6.7]. Since $X_{n}=\operatorname{Proj} S$ for $S=\bigoplus J^{k} \delta^{k}$, in the notation of (3.7.1), this implies that $\widehat{\Phi}\left(H_{c}\right) \cong \pi(S)=\mathcal{P}$, as required.

4.6. Recall from (2.6) the definitions of the the characteristic variety Char $M \subseteq \operatorname{Hilb}(\mathrm{n})$ and the associated variety $\operatorname{Char}_{0} M \subseteq \mathfrak{h} \oplus \mathfrak{h}^{*} / W$ for $(M, \Lambda) \in U_{c^{-}}$filt. As has been mentioned in the introduction, Char $M$ carries more subtle information $M$ than $\mathrm{Char}_{0} M$. Nevertheless these two varieties are connected and we will show in Proposition 4.8 that $\operatorname{Char}_{0} M=\tau(\operatorname{Char} M)$, under the resolution of singularities $\tau$. In a weak 
sense this shows that the natural diagram

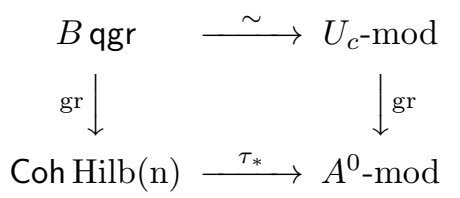

commutes. One can show by example that the stronger commutativity result, $\tau_{*}\left(\operatorname{gr}_{\Lambda}(\widetilde{M})\right)=\operatorname{gr}_{\Lambda}(M)$, does not always hold.

We begin with an abstract lemma.

Lemma. Let $R=\bigcup F^{i} R$ and $S=\bigcup F^{i} S$ be subrings of a filtered ring $D=\bigcup_{i \geq 0} F^{i} D$ and $T$ an $(S, R)$-subbimodule of D. Assume that $\operatorname{gr}_{F} D$ is commutative, that $\operatorname{gr}_{F} R=\operatorname{gr}_{F} S$ under the induced filtrations and that the induced filtration $F$ on $T$ is good on both sides. Let $(N, F) \in R$-filt and give $T \otimes_{R} N$ the tensor product filtration $F$. Then $I=\operatorname{ann}_{\operatorname{gr}_{F} R}\left(\operatorname{gr}_{F} N\right) \subseteq \operatorname{ann}_{\operatorname{gr}_{F}}\left(\operatorname{gr}_{F}(T \otimes N)\right)$.

Proof. We drop the subscript $F$ from all graded modules. The commutativity of gr $D$ implies the following: take $\bar{q} \in \operatorname{gr}^{j} T$ and $\bar{r} \in \operatorname{gr}^{i} R=\operatorname{gr}^{i} S$, and lift $\bar{q}$ to $q \in F^{j} T$ and lift $\bar{r}$ to elements $r_{1} \in F^{i} S$ and $r_{2} \in F^{i} R$. Then, $r_{1} q=q r_{2}$ modulo $F^{i+j-1} T$.

Recall from (2.4) that the principal symbol of $n \in N$ is written $\sigma(n)$. Now let $\bar{a} \in I_{k}=I \cap \operatorname{gr}^{k} R$ and pick a typical generator, say $\sigma(q \otimes n)$ of $\operatorname{gr}^{t}(T \otimes N)$, where $q \in F^{i} T$ and $n \in F^{t-i} N$. Lift $\bar{a}$ to elements $a \in F^{k} S$ and $b \in F^{k} R$. Since $\sigma(b) \sigma(n)=0$, clearly $b n \in F^{k+(t-i-1)}(N)$. By the last paragraph, $a q-q b=c \in F^{k+i-1} T$ and so

$$
a(q \otimes n)=q b \otimes n+c \otimes n=q \otimes b n+c \otimes n \in F^{k+t-1}(T \otimes N)
$$

In other words, $\bar{a} \sigma(q \otimes n)=0$ and hence $\bar{a} \operatorname{gr}(T \otimes N)=0$.

4.7. Corollary. Let $R_{\mathbb{Z}}$ be a Morita $\mathbb{Z}$-algebra satisfying Hypotheses (1-4) of (2.3) and suppose that $M$ is a finitely generated left $R_{0}$-module. Then $\operatorname{Char}_{0} M=\operatorname{Char}_{0}\left(R_{k 0} \otimes_{R_{0}} M\right)$ for all $k \geq 0$.

Proof. Set $Q=R_{k 0}$. Pick a good filtration $\Lambda$ of $M$ and give $Q \otimes M$ the tensor product filtration $\Gamma$; by [GS, Lemma 6.7(2)] this is a good filtration. Since Char $_{0} M$ is independent of the choice of good filtration [Bj2, Proposition 5.1], it is determined by $\operatorname{ann}_{\operatorname{gr}\left(R_{0}\right)}\left(\operatorname{gr}_{\Lambda} M\right)$. The hypotheses of Lemma 4.6 are satisfied by $R=R_{0}, S=R_{k}, T=Q$ and $N=M$ and so that lemma implies that $\operatorname{Char}_{0} M \supseteq \operatorname{Char}_{0}(Q \otimes M)$.

The lemma may also be applied to the rings $R=R_{k}, S=R_{0}$ with the modules $T=P=Q^{*}$ and $N=Q \otimes M$, with its good tensor product filtration $\Gamma$. By hypothesis, $Q$ is a progenerator and so $P \otimes_{R_{k}} N=$ $\left(P \otimes_{R_{k}} Q\right) \otimes_{R_{0}} M \cong M$. Thus the lemma now implies that $\operatorname{Char}_{0}(Q \otimes M)=\operatorname{Char}_{0} N \supseteq \operatorname{Char}_{0}(P \otimes N)=$ $\operatorname{Char}_{0} M$ and hence that $\operatorname{Char}_{0} M=\operatorname{Char}_{0}(Q \otimes M)$. 
4.8. We can now relate $\operatorname{Char}_{0} M$ to Char $M$ for a finitely generated $U_{c}$-module $M$, where $c$ satisfies Hypothesis 3.4. We will also need this result in other contexts and so we generalise it slightly. Let $R_{\mathbb{Z}}$ be a Morita $\mathbb{Z}$-algebra that satisfies Hypotheses (1-4) of (2.3). The graded ring $S=\bigoplus S_{i}$ of (2.3)(4) satisfies $S_{0}=\operatorname{gr} R_{0}$ and so we have a natural surjection $\tau^{\prime}: \operatorname{Proj} S \rightarrow \operatorname{Spec} \operatorname{gr} R_{0}$ analogous to $\tau$.

Proposition. Let $R_{\mathbb{Z}}$ be a Morita $\mathbb{Z}$-algebra that satisfies Hypotheses (1-4) of (2.3) and let $M$ be a finitely generated left $R_{0}$-module. Then $\tau^{\prime}(\mathbf{C h a r} M)=\operatorname{Char}_{0} M$.

Remark. As was remarked in [GS, (1.3)], Theorem 1.2 can be regarded as a kind of analogue for Cherednik algebras of the Beilinson-Bernstein equivalence of categories for enveloping algebras. Under this analogy, the proposition for $U_{c}$ corresponds to [BB, Theorem 1.9(c)].

Proof. Identify $S_{0}=\operatorname{gr} R_{0}$ and set $I=\operatorname{ann}_{S_{0}}(\operatorname{gr} M)$ and $J=\operatorname{ann}_{S}(\operatorname{gr} \widetilde{M})$. If $P=\bigoplus_{i \geq 0} P_{i}$ is a graded prime ideal of $S$ that does not contain the irrelevant ideal $S_{+}=\bigoplus_{i>0} S_{i}$, then the corresponding varieties satisfy $\tau^{\prime}(\mathcal{V}(P))=\mathcal{V}\left(P_{0}\right)$. For an arbitrary graded ideal of $S$ we have to worry about occurrences of $S_{+}$. Thus, let $K=\bigoplus_{i \geq 0} K_{i}$ denote the largest ideal $L$ of $S$ such that $L\left(S_{+}\right)^{m} \subseteq J$ for some $m \geq 0$. By definition $\mathcal{V}(J)=$ $\mathcal{V}(K)$ but now the prime ideals $P$ minimal over $K$ do not contain $S_{+}$. Thus $\tau^{\prime}(\mathcal{V}(J))=\tau^{\prime}(\mathcal{V}(K))=\mathcal{V}\left(K_{0}\right)$.

A standard exercise shows that $K=\operatorname{ann}_{S}\left((\operatorname{gr} \widetilde{M})_{\geq t}\right)$, for $t \gg 0$. For some such $t$ pick such that $(\operatorname{gr} \widetilde{M})_{\geq t}$ is generated by $\bigoplus_{j=t}^{s} \operatorname{gr} M(j)$ as an $S$-module. Clearly $K_{0}=\bigcap_{j=t}^{s} \operatorname{ann}_{S_{0}} \operatorname{gr} M(j)$. By Corollary 4.7 $\sqrt{I}=\sqrt{\operatorname{ann}_{S_{0}}(\operatorname{gr} M(j))}$ for each such $j$ and so $\sqrt{I}=\sqrt{K_{0}}$. By the conclusion of the last paragraph, this implies that $\tau^{\prime}(\mathbf{C h a r} M)=\operatorname{Char}_{0} M$.

4.9. Let $M \in U_{c}$-mod be pure in the sense that each nonzero submodule has the same Gelfand-Kirillov dimension as $M$. By Watanabe's Theorem [Wt], $\mathbb{C}\left[\mathfrak{h} \oplus \mathfrak{h}^{*}\right]^{W}$ is Gorenstein and so Gabber's theorem says that the associated variety $\mathrm{Char}_{0} M$ is equidimensional (see, for example, [Bj2, (5.2)] and [Bj1, Theorem 7.1]). It would be interesting to know if the analogous result holds for Char:

Question. If $M \in U_{c}$-mod is pure, is Char $M$ equidimensional?

4.10. The BKR equivalence. In the final result of this section, Proposition 4.11, we show that there is an interesting connection between $\widehat{\Phi}$ and the Bridgeland-King-Reid equivalence of categories.

We start by giving a version of that equivalence of categories that is appropriate for $\mathfrak{h} \oplus \mathfrak{h}^{*} / W$. Recall the following commutative diagram from (3.7.2).

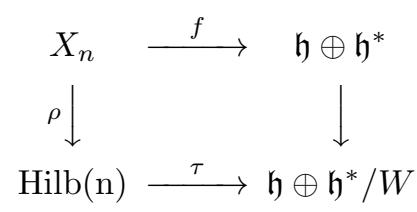

Let $D(\operatorname{Hilb}(\mathrm{n}))$ denote the derived category of complexes of quasicoherent sheaves on Hilb(n) with bounded coherent cohomology and let $D^{W}\left(\mathfrak{h} \oplus \mathfrak{h}^{*}\right)$ be the derived category of complexes of $W$-equivariant quasicoherent 
sheaves on $\mathfrak{h} \oplus \mathfrak{h}^{*}$, again with bounded coherent cohomology. Equivalently, $D^{W}\left(\mathfrak{h} \oplus \mathfrak{h}^{*}\right)$ can be identified with the derived category of bounded complexes of finitely generated $\mathbb{C}\left[\mathfrak{h} \oplus \mathfrak{h}^{*}\right] * W$-modules.

Theorem. ([BKR, Theorem 1.1] and [Ha3, Corollary 5.1]) The functor $\Psi: D^{W}\left(\mathfrak{h} \oplus \mathfrak{h}^{*}\right) \rightarrow D(H i l b(\mathrm{n}))$ given by $\mathcal{F} \mapsto\left(\rho_{*} \circ L f^{*}(\mathcal{F})\right)^{W}$ is an equivalence of triangulated categories.

Proof. By [GS, Corollary 4.10], $\tau: \operatorname{Hilb}(\mathrm{n}) \rightarrow \mathfrak{h} \oplus \mathfrak{h}^{*} / W$ is a crepant resolution of singularities and Hilb(n) is irreducible. Since $W$ preserves the symplectic form on $\mathfrak{h} \oplus \mathfrak{h}^{*}$, the result will therefore follow from [BKR, Corollary 1.3] once we show that $\operatorname{Hilb}(\mathrm{n}) \cong \mathfrak{h} \oplus \mathfrak{h}^{*} / / W$, the $W$-Hilbert scheme on $\mathfrak{h} \oplus \mathfrak{h}^{*}$. By definition the points of $\mathfrak{h} \oplus \mathfrak{h}^{*} / / W$ are (isomorphism classes of) $W$-clusters on $\mathfrak{h} \oplus \mathfrak{h}^{*}$; that is, cyclic $\mathbb{C}\left[\mathfrak{h} \oplus \mathfrak{h}^{*}\right] * W$-modules which both carry the regular representation as $W$-modules and are generated by the trivial representation.

Let $\mathbb{C}^{2 n} / / W$ denote the $W$-Hilbert scheme on $\mathbb{C}^{2 n}$. Any central element of $\mathbb{C}\left[\mathbb{C}^{2 n}\right] * W$ acts by scalar multiplication on a $W$-cluster in $\mathbb{C}^{2 n}$, so there is a morphism $p: \mathbb{C}^{2 n} / / W \longrightarrow S^{n} \mathbb{C}^{2}$. Since $\mathfrak{h} \oplus \mathfrak{h}^{*} / / W$ consists of the clusters which are annihilated by the elements $\mathbf{z}$ and $\mathbf{z}^{*}$ of $(3.6)$, it follows that $p^{-1}\left(\mathfrak{h} \oplus \mathfrak{h}^{*} / W\right)=$ $\mathfrak{h} \oplus \mathfrak{h}^{*} / / W$. By [Ha2, Theorem 5.1] there is an isomorphism of $S^{n} \mathbb{C}^{2}$-varieties $\iota: \mathbb{C}^{2 n} / / W \longrightarrow$ Hilb $^{n} \mathbb{C}^{2}$. Since $\operatorname{Hilb}(\mathrm{n})=\tau^{-1}\left(\mathfrak{h} \oplus \mathfrak{h}^{*} / W\right)$ this induces the required isomorphism $\mathfrak{h} \oplus \mathfrak{h}^{*} / / W \cong \operatorname{Hilb}(\mathrm{n})$.

Finally, we compute the functor giving the equivalence of categories, since the functor we want is not quite that of [BKR] or [Ha3]. As is explained by [Ha3, Corollary 5.1 and Proposition 5.3], adjusted to our setup over $\mathfrak{h} \oplus \mathfrak{h}^{*}$, the equivalence $D(\operatorname{Hilb}(\mathrm{n})) \rightarrow D^{W}\left(\mathfrak{h} \oplus \mathfrak{h}^{*}\right)$ is given by $\alpha=R \Gamma_{X_{n}}(\mathcal{P} \otimes-)$, with inverse $\mathcal{O}(1) \otimes\left(\rho_{*} \circ L f^{*}\right)^{\text {sign }}$. Consequently, if we replace $\alpha$ by the functor $R \Gamma_{X_{n}}(\mathcal{P}(-1) \otimes-) \otimes$ sign, which is obviously still an equivalence, then its inverse will indeed be the functor $\left(\rho_{*} \circ L f^{*}\right)^{W}$ that we require.

4.11. Theorem 4.10 gives a second way in which to construct coherent sheaves on Hilb(n) from objects of $H_{c^{-}}$filt. Let $(N, \Gamma) \in H_{c^{-}}$-filt. Then $\operatorname{gr}_{\Gamma} N$ is a finitely generated $\mathbb{C}\left[\mathfrak{h} \oplus \mathfrak{h}^{*}\right] * W$-module, which we consider as an object of $D^{W}\left(\mathfrak{h} \oplus \mathfrak{h}^{*}\right)$ concentrated in degree zero. Applying the functor of Theorem 4.10 to this module gives a complex $\Psi(N)=\Psi_{\Gamma}(N)$ of coherent sheaves on Hilb(n). Finally, taking the zeroth homology of $\Psi$ gives the functor

$$
\Psi_{\Gamma}^{0}: H_{c} \text {-filt } \longrightarrow \text { Coh Hilb(n), } \quad(N, \Gamma) \mapsto\left(\rho_{*} f^{*}\left(\operatorname{gr}_{\Gamma} N\right)\right)^{W} .
$$

The following proposition shows this functor is closely related to the functor $\widehat{\Phi}$ obtained from our $\mathbb{Z}$-algebra construction.

Proposition. For every $(N, \Gamma) \in H_{c}$-filt there is a surjective mapping $\Psi_{\Gamma}^{0}(N) \rightarrow \widehat{\Phi}_{\Gamma}(N)$ of sheaves on $\operatorname{Hilb}(n)$.

Proof. To prove the proposition we will first study free $H_{c}$-modules, and then pass to the general case via free $H_{c}$-resolutions. The result for free modules follows from the following result.

Sublemma. Let $R_{x}: H_{c} \rightarrow H_{c}$ be the mapping given by right multiplication by an element $x \in H_{c}$. Then $\Psi_{\text {ord }}^{0}\left(H_{c}\right)=\mathcal{P}=\widehat{\Phi}_{\text {ord }}\left(H_{c}\right)$ and the induced map $\Psi_{\text {ord }}^{0}\left(R_{x}\right): \mathcal{P} \rightarrow \mathcal{P}$ coincides with $\widehat{\Psi}\left(R_{x}\right)$. 
Proof of the sublemma. This just amounts to unravelling the definitions and we begin with $\widehat{\Psi}$.

Recall from (4.3) that the ord and tensor product filtrations coincide on $\widetilde{e H}_{c}$. It is therefore clear that the map ogr $R_{x}:$ ogr $\widetilde{e H}_{c} \rightarrow$ ogr $\widetilde{e H}_{c}$ induced from $R_{x}$ is just given by right multiplication by the principal symbol $\sigma(x) \in \mathbb{C}\left[\mathfrak{h} \oplus \mathfrak{h}^{*}\right] * W$ of $x$. Write $\sigma(x)=\sum p_{\gamma} \gamma$, for some $p_{\gamma} \in \mathbb{C}\left[\mathfrak{h} \oplus \mathfrak{h}^{*}\right]$ and $\gamma \in W$ and recall from (3.1) that multiplication in $\mathbb{C}\left[\mathfrak{h} \oplus \mathfrak{h}^{*}\right] * W$ is defined by $w f=w(f) w$ for $w \in W$ and $f \in \mathbb{C}\left[\mathfrak{h} \oplus \mathfrak{h}^{*}\right]$. By Theorem 4.5, ogr $\widetilde{e H}_{c} \cong S=\bigoplus_{k \geq 0} J^{k} \delta^{k}$, where the isomorphism is induced on each summand from the natural isomorphism $\Theta^{-1}: e\left(\mathbb{C}\left[\mathfrak{h} \oplus \mathfrak{h}^{*}\right] * W\right) \stackrel{\sim}{\rightarrow} \mathbb{C}\left[\mathfrak{h} \oplus \mathfrak{h}^{*}\right]$ given by $e p \gamma=e \gamma \gamma^{-1}(p) \mapsto \gamma^{-1}(p)$, for $p \in \mathbb{C}\left[\mathfrak{h} \oplus \mathfrak{h}^{*}\right]$ and $\gamma \in W$. Therefore, at the level of graded $A$-modules, the map $\widehat{\Phi}\left(R_{x}\right)$ is the map $S \rightarrow S$ defined by

$$
j \mapsto e j \mapsto \sum_{\gamma} e j p_{\gamma} \gamma \mapsto \sum_{\gamma} \gamma^{-1}\left(j p_{\gamma}\right), \quad \text { for } j \in J^{k} \delta^{k} .
$$

Now consider $\Psi^{0}$. The map $f: X_{n} \longrightarrow \mathfrak{h} \oplus \mathfrak{h}^{*}$ is $W$-equivariant, so the pullback $f^{*}$ sends $W$-equivariant coherent sheaves on $\mathfrak{h} \oplus \mathfrak{h}^{*}$ to $W$-equivariant coherent sheaves on $X_{n}$. We identify the category of $W$ equivariant coherent sheaves on $\mathfrak{h} \oplus \mathfrak{h}^{*}$ with $\mathbb{C}\left[\mathfrak{h} \oplus \mathfrak{h}^{*}\right] * W$-mod and, by (3.7), write $X_{n}=\operatorname{Proj} S$. In this language, if $M$ is an object of $\mathbb{C}\left[\mathfrak{h} \oplus \mathfrak{h}^{*}\right] * W$-mod, then $f^{*}(M)=S \otimes_{\mathbb{C}\left[\mathfrak{h} \oplus \mathfrak{h}^{*}\right]} M$ with $W$ acting diagonally on the right hand side. Similarly, the functor $\rho_{*}: \operatorname{Coh} X_{n} \rightarrow \operatorname{Coh} \operatorname{Hilb}(\mathrm{n})$ corresponds to the restriction functor from graded $S$-modules to graded $A$-modules.

The map ogr $R_{x}: \operatorname{ogr} H_{c}=\mathbb{C}\left[\mathfrak{h} \oplus \mathfrak{h}^{*}\right] * W \rightarrow \mathbb{C}\left[\mathfrak{h} \oplus \mathfrak{h}^{*}\right] * W$ is clearly given by right multiplication by $\sigma(x)$ and so, by the last paragraph, $\Psi^{0}\left(R_{x}\right)$ is given by the homomorphism of graded $A$-modules

$$
R_{\sigma(x)}:\left(S \otimes_{\mathbb{C}\left[\mathfrak{h} \oplus \mathfrak{h}^{*}\right]}\left(\mathbb{C}\left[\mathfrak{h} \oplus \mathfrak{h}^{*}\right] * W\right)\right)^{W} \longrightarrow\left(S \otimes_{\mathbb{C}\left[\mathfrak{h} \oplus \mathfrak{h}^{*}\right]}\left(\mathbb{C}\left[\mathfrak{h} \oplus \mathfrak{h}^{*}\right] * W\right)\right)^{W}
$$

defined by right multiplication by $\sigma(x)$. We need to compare this to (4.11.1). There is a natural identification

$$
\chi: S \stackrel{\sim}{\longrightarrow}\left(S \otimes_{\mathbb{C}} \mathbb{C} W\right)^{W} \stackrel{\sim}{\longrightarrow}\left(S \otimes_{\mathbb{C}\left[\mathfrak{h} \oplus \mathfrak{h}^{*}\right]}\left(\mathbb{C}\left[\mathfrak{h} \oplus \mathfrak{h}^{*}\right] * W\right)\right)^{W}
$$

given by

$$
b \mapsto \frac{1}{n !} \sum_{g \in W} g(b) \otimes_{\mathbb{C}} g \mapsto \frac{1}{n !} \sum_{g \in W} g(b) \otimes_{\mathbb{C}\left[\mathfrak{h} \oplus \mathfrak{h}^{*}\right]} g \quad \text { for } b \in S .
$$

Write $\sigma(x)=\sum p_{\gamma} \gamma$, as before. Composing the map $\chi$ with (4.11.2) yields the mapping $S \rightarrow S$ given by

$$
\begin{aligned}
b \mapsto \frac{1}{n !} \sum_{g} g(b) \otimes g & \stackrel{\cdot \sigma(x)}{\longmapsto} \frac{1}{n !} \sum_{g, \gamma} g(b) \otimes g p_{\gamma} \gamma \\
& =\frac{1}{n !} \sum_{g, \gamma} g \gamma\left(\gamma^{-1}\left(b p_{\gamma}\right)\right) \otimes g \gamma \mapsto \sum_{\gamma} \gamma^{-1}\left(b p_{\gamma}\right) .
\end{aligned}
$$

Thus $\Psi^{0}\left(\operatorname{ogr} R_{x}\right)$ coincides with $\widehat{\Phi}\left(R_{x}\right)$ as graded $A$-module maps from $S$ to $S$. As in the proof of Theorem 4.5, $\pi(S)=\mathcal{P} \in \operatorname{Coh} \operatorname{Hilb}(\mathrm{n})$ and so the sublemma follows.

We return to the proof of the proposition and let $(N, \Gamma) \in H_{c^{-}}$filt. By [Bj1, Section 2.3.11], we can construct an exact sequence in $H_{c}$-filt

$$
\cdots \stackrel{d}{\longrightarrow} F_{i} \stackrel{d}{\longrightarrow} F_{i-1} \underset{23}{\longrightarrow} \cdots \stackrel{d}{\longrightarrow} F_{0} \stackrel{\eta}{\longrightarrow} N \longrightarrow 0
$$


where the $F_{i}$ are filtered free $H_{c}$-modules whose filtrations shift the ord filtration and the induced complex

$$
\cdots \stackrel{\operatorname{gr} d}{\longrightarrow} \operatorname{gr} F_{i} \stackrel{\operatorname{gr} d}{\longrightarrow} \operatorname{gr} F_{i-1} \stackrel{\text { gr } d}{\longrightarrow} \cdots \stackrel{\text { gr } d}{\longrightarrow} \operatorname{gr} F_{0} \stackrel{\text { gr } \eta}{\longrightarrow} \operatorname{gr}_{\Gamma} N \longrightarrow 0
$$

is a free resolution of $\operatorname{gr}_{\Gamma} N$. In particular the maps $d$ are given by right multiplication by matrices with entries from $H_{c}$ and so the sublemma can be applied to them. By Theorem 4.5, if we apply $\widehat{\Phi}$ to (4.11.3) we obtain the complex of coherent sheaves on $\operatorname{Hilb}(\mathrm{n})$

$$
\cdots \stackrel{\operatorname{gr} \tilde{d}}{\longrightarrow} \mathcal{P}^{\oplus n_{i}} \stackrel{\operatorname{gr} \tilde{d}}{\longrightarrow} \mathcal{P}^{\oplus n_{i-1}} \stackrel{\operatorname{gr} \tilde{d}}{\longrightarrow} \cdots \stackrel{\text { gr } \tilde{d}}{\longrightarrow} \mathcal{P}^{\oplus n_{0}} \stackrel{\text { gr } \tilde{\eta}}{\longrightarrow} \widehat{\Phi}(N) \longrightarrow 0,
$$

where $n_{i}$ is the rank of $F_{i}$ over $H_{c}$ and gr $\tilde{d}$ is obtained from $d$ by repeated use of (4.11.1). As in the proof of Lemma 2.5(1), the surjectivity of $\eta$ implies that final map gr $\tilde{\eta}$ in (4.11.5) is also surjective. Unfortunately, as happened in the proof of Lemma 2.8, one cannot expect that gr $\tilde{d}$ is right exact and so we can only conclude that the zeroth cohomology of the deleted complex (4.11.5) surjects onto $\widehat{\Phi}(N)$.

On the other hand, $\left(\rho_{*} f^{*}(-)\right)^{W}$ is a right exact functor and so $\Psi^{0}(N)$ is the zeroth cohomology of the functor $\left(\rho_{*} f^{*}(-)\right)^{W}$ applied to the deleted complex (4.11.4). By the sublemma, this new complex is just the deleted complex (4.11.5). The proposition follows.

4.12. It is natural to ask whether Proposition 4.11 is the shadow of a much stronger statement.

Question 1. Let $(N, \Gamma) \in H_{c}$-filt. Is there a quasi-isomorphism of coherent sheaves between $\widehat{\Phi}_{\Gamma}(N)$ and $\Psi_{\Gamma}(N)$ ? In other words is $\Psi_{\Gamma}^{0}(N) \cong \widehat{\Phi}_{\Gamma}(N)$ and do the homology sheaves $\mathrm{H}^{i}\left(\Psi_{\Gamma}(N)\right)$ vanish for $i>0$ ?

It is instructive to rephrase the final part of this question. As we observed in the proof of Theorem 4.10, the inverse to $\Psi$ is the functor $R \Gamma(\mathcal{P}(-1) \otimes-) \otimes$ sign. By [Ha2, (45)] $\mathcal{P}(-1) \cong \mathcal{P}^{*}$, the dual bundle of $\mathcal{P}$. Thus the following question is a special case of Question 1.

Question 2. If $(N, \Gamma) \in H_{c}$-mod, does $\mathrm{H}^{i}\left(\operatorname{Hilb}(\mathrm{n}), \mathcal{P}^{*} \otimes \widehat{\Phi}_{\Gamma}(N)\right)=0$ for $i>0$ ?

This question is rather similar to [Ha3, Conjecture 3.2] which asks the following: Let $\mathcal{B}_{1}$ denote the tautological rank $n$ bundle and $\mathcal{P}_{1}$ the Procesi bundle on $\operatorname{Hilb}^{\mathrm{n}} \mathbb{C}^{2}$, as in the proof of Lemma 3.12. Then:

Conjecture. [Ha3] Does $\mathrm{H}^{i}\left(\mathrm{Hilb}^{\mathrm{n}} \mathbb{C}^{2}, \mathcal{P}_{1}^{*} \otimes \mathcal{B}_{1}^{\otimes \ell}\right)=0$ for all $i>0$ and all $\ell \geq 0$.

\section{Finite dimensional $U_{c}$-Modules and their Characteristic VARieties}

5.1. We assume throughout the section that $c \in \mathbb{C}$ satisfies Hypothesis 3.4. Some of the most interesting $H_{c^{-}}$ modules are the finite dimensional ones, which exist if (and only if) $c=r / n$, for some $r \in \mathbb{N}$ with $(r, n)=1$ [BEG2, Theorem 1.2]. For such $c$, the irreducible finite dimensional modules are just the modules $L_{c}($ triv), as defined below in (5.2). In particular the module $L_{1 / n}$ (triv) is one-dimensional (see [BEG2, Proposition 2.1]) and each $L_{1 / n+k}$ (triv) is induced from $L_{1 / n}$ (triv) via the shift functors $\widetilde{S}$.

Our $\mathbb{Z}$-algebra techniques are particularly well suited to studying these modules and we give the details in this section. We first prove in Proposition 5.4 that $\widehat{\Phi}\left(L_{1 / n}\right.$ (triv)) is simply $\mathcal{O}_{Z_{n}}$, where $Z_{n}$ is the punctual 
Hilbert scheme. This statement contains within it information about each $L_{1 / n+k}($ triv) and this allows us to answer conjectures of Berest, Etingof and Ginzburg from [BEG2, Section 7]. Essentially, these questions ask for a bigraded decomposition of the associated graded modules of $L_{1 / n+k}$ (triv) and $e L_{1 / n+k}$ (triv). However, the formal statement uses slightly different filtrations from the ones use so far and so we refer the reader to Theorem 5.11 for the precise statement.

5.2. Category $\mathcal{O}_{c}$. As in [GGOR] or [BEG1, Definition 2.4], we define $\mathcal{O}_{c}$ to be the abelian category of finitely-generated $H_{c}$-modules $M$ for which the action of $\mathbb{C}\left[\mathfrak{h}^{*}\right]$ on $M$ is locally nilpotent. By [Gu, Theorem 3] $\mathcal{O}_{c}$ is a highest weight category. Given $\mu \in \operatorname{Irrep}(W)$, we define the standard module $\Delta_{c}(\mu) \in \mathcal{O}_{c}$, to be the induced module

$$
\Delta_{c}(\mu)=H_{c} \otimes_{\mathbb{C}\left[\mathfrak{h}^{*}\right] * W} \mu
$$

where $\mathbb{C}\left[\mathfrak{h}^{*}\right] * W$ acts on $\mu$ by $p w \cdot m=p(0)(w \cdot m)$, for $p \in \mathbb{C}\left[\mathfrak{h}^{*}\right], w \in W$ and $m \in \mu$. It follows from the PBW Theorem 3.2.1 that $\Delta_{c}(\mu)$ is a free left $\mathbb{C}[\mathfrak{h}]$-module of rank $\operatorname{dim}(\mu)$. It is shown in [BEG1, Section 2] that each $\Delta_{c}(\mu)$ has a unique simple quotient $L_{c}(\mu)$, that the set $\left\{L_{c}(\mu): \mu \in \operatorname{Irrep}(W)\right\}$ provides a complete list of non-isomorphic simple objects in $\mathcal{O}_{c}$, and that every object in $\mathcal{O}_{c}$ has finite length.

5.3. The following observation will be used several times.

Lemma. Let $U^{\prime}$ and $U$ be subrings of a filtered $\mathbb{C}$-algebra $D=\bigcup_{i \geq 0} F^{i} D$ and $T$ a $\left(U^{\prime}, U\right)$-submodule of $D$. Give $U^{\prime}, U$ and $T$ the induced filtration and suppose that $U$ has a 1-dimensional module $\mathbb{C}=U / \mathfrak{n}$ with the trivial filtration $F^{r}(\mathbb{C})=F^{0}(\mathbb{C})=\mathbb{C}$ for all $r \geq 0$. Then, under the tensor product filtration on $T \otimes_{U} \mathbb{C}$, we have $F^{i}(T \otimes \mathbb{C})=\left(F^{i} T\right) \otimes \mathbb{C}$ and a surjective $\operatorname{gr}_{F} U^{\prime}$-module map $\operatorname{gr}_{F} T / \operatorname{gr}_{F} T \operatorname{gr}_{F} \mathfrak{n} \rightarrow \operatorname{gr}_{F}(T \otimes \mathbb{C})$.

Proof. Clearly $F^{i}(T \otimes \mathbb{C})=\sum_{r} F^{i-r} T \otimes F^{r}(\mathbb{C})=F^{i}(T) \otimes \mathbb{C}=\left(F^{i} T+T \mathfrak{n}\right) / T \mathfrak{n}$. This induces a surjective $\operatorname{map} F^{i} T / F^{i-1} T \rightarrow\left(F^{i} T+T \mathfrak{n}\right) /\left(F^{i-1} T+T \mathfrak{n}\right)=F^{i}(T \otimes \mathbb{C}) / F^{i-1}(T \otimes \mathbb{C})$, and hence a surjection $\operatorname{gr}_{F} T \rightarrow$ $\operatorname{gr}_{F}(T \otimes \mathbb{C})$. The kernel of this map contains $\operatorname{gr}_{F}(T \mathfrak{n}) \supseteq \operatorname{gr}_{F}(T) \operatorname{gr}_{F}(\mathfrak{n})$.

5.4. Let $c=1 / n$. By [BEG2, Proposition 2.1] or [G2, Lemma 4.6] the $H_{c}$-module $L_{c}$ (triv) is one dimensional. Thus, if we give $L_{c}$ (triv) the trivial filtration $\Gamma^{i}\left(L_{c}\right.$ (triv) $)=L_{c}$ (triv) for $i \geq 0$, then $\operatorname{gr}_{\Gamma} L_{c}($ triv $)=\mathbb{C}$ is the trivial $\mathbb{C}\left[\mathfrak{h} \oplus \mathfrak{h}^{*}\right] * W$-module concentrated in degree 0 . The first main result of this section identifies the $\operatorname{Hilb}(\mathrm{n})$-module $\widehat{\Phi}\left(L_{c}(\right.$ triv $\left.)\right)$.

Proposition. Let $c=1 / n$ and give $L_{c}($ triv $) \cong \mathbb{C}$ the trivial filtration $\Gamma$. Then $\widehat{\Phi}_{\Gamma}\left(L_{c}(\right.$ triv $\left.)\right) \cong \mathcal{O}_{Z_{\mathrm{n}}}$.

Proof. Recall that $A=\bigoplus_{k \geq 0} A^{k} \delta^{k}$. Identify $e L_{c}$ (triv) with $N=U_{c} / \widehat{\mathfrak{m}}$ for the appropriate ideal $\widehat{\mathfrak{m}}$. Filter $N$ by $F^{0}(N)=N$, and write $N(k)=B_{k 0} \otimes_{U_{c}} N$ as in (2.4). By [BEG1, Remark, p.306] $\mathfrak{h}$ and $\mathfrak{h}^{*}$ annihilate $L_{c}$ (triv), so $\mathfrak{m}=\operatorname{ogr}(\widehat{\mathfrak{m}})=\mathbb{C}\left[\mathfrak{h} \oplus \mathfrak{h}^{*}\right]_{+}^{W}$, the maximal ideal of $A^{0}=\mathbb{C}\left[\mathfrak{h} \oplus \mathfrak{h}^{*}\right]^{W}$ corresponding to the zero orbit. For any $k \geq 0,(3.9 .1)$ and Lemma 5.3 combine to give a surjection

$$
\eta_{k}: \frac{A^{k} \delta^{k}}{A^{k} \delta^{k} \mathfrak{m}} \cong \frac{\operatorname{ogr} B_{k 0}}{\underset{25}{\operatorname{ogr} B_{k 0} \operatorname{ogr} \widehat{\mathfrak{m}}}} \rightarrow \operatorname{gr} N(k)
$$


and, hence, a surjection $\eta: A / A \mathfrak{m} \rightarrow \operatorname{gr}(\widetilde{N})$. On the other hand, Lemma 3.12 shows that $\mathrm{H}^{0}\left(\mathrm{Z}_{\mathrm{n}}, \mathcal{L}^{k}\right) \cong$ $A^{k} \delta^{k} / A^{k} \delta^{k} \mathfrak{m}$ and so $\mathcal{O}_{\mathrm{Z}_{\mathrm{n}}}$ is the Hilb(n)-module corresponding to the graded $A$-module $A / A \mathfrak{m}$.

In order to complete the proof of the proposition it therefore suffices to show that $\eta$ is an isomorphism, which we do by computing dimensions. By comparing Lemma 3.15 and [BEG2, Theorem 1.14] we obtain

$$
\operatorname{dim} A^{k} \delta^{k} / A^{k} \delta^{k} \mathfrak{m}=\frac{1}{k n+1}\left(\begin{array}{c}
(k+1) n \\
n
\end{array}\right)=\operatorname{dim} e L_{c+k}(\operatorname{triv})=\operatorname{dim} N(k) .
$$

Since the maps $\eta_{k}$ are surjections they are therefore isomorphisms. Hence, so is $\eta$.

5.5. As remarked in (5.1), the simple module $L_{c}$ (triv) is finite dimensional whenever $c=r / n$ with $r \in \mathbb{N}$ and $(r, n)=1$. It would be interesting to know whether an analogue of Proposition 5.4 holds for these modules:

Problem. If $c=r / n$ with $(r, n)=1$, find $\widehat{\Phi}\left(L_{c}(\right.$ triv $\left.)\right)$ under some natural filtration on $L_{c}($ triv).

A shadow of the answer will be given in (6.9) which computes the restricted character of $L_{c}$ (triv).

5.6. By [GS, Proposition 3.16], $B_{k 0} \otimes_{U_{c}} e L_{c}$ (triv) $\cong e L_{c+k}$ (triv) for $k>0$ and so Proposition 5.4 also contains information about the modules $e L_{1 / n+k}$ (triv). For the rest of this section we will study the consequences of this observation. In particular we will determine the bigraded structure of the associated graded modules of these modules, thereby answering the conjectures from [BEG2, Section 7].

5.7. Torus action revisited. As we will discuss, this bigraded structure is the standard one for rings of differential operators, but it is not quite the bigrading used in (3.13) and [Ha3]. Instead, we need to consider a second action of $\mathbb{T}^{2}$ on $\mathbb{C}\left[\mathbb{C}^{2}\right]=\mathbb{C}[x, y]$, this time given by $\tau_{s, t} \cdot x=s t x, \tau_{s, t} \cdot y=s t^{-1} y$. This induces new $\mathbb{T}^{2}$-structures on $\operatorname{Hilb}(\mathrm{n}), \mathrm{Z}_{\mathrm{n}}, \mathcal{P}, \mathcal{L}$, etc.

It is routine to check that, if an element $f$ is bihomogeneous of weight $(i, j)$ for the action in (3.13), then under this new action $\tau_{s, t} \cdot f=s^{i+j} t^{i-j} f$ and so $f$ is bihomogeneous of weight $(i+j, i-j)$. Thus, under the present torus action, Lemma 3.14 provides the following identification of bigraded components:

$$
\mathrm{H}^{0}\left(\mathrm{Z}_{\mathrm{n}}, \mathcal{L}^{k}\right)^{i j} \cong\left(\frac{A^{k} \delta^{k}}{A^{k} \delta^{k} \mathfrak{m}}\right)^{i+N k, j+N k}, \quad \mathrm{H}^{0}\left(\mathrm{Z}_{\mathrm{n}}, \mathcal{P} \otimes \mathcal{L}^{k}\right)^{i j} \cong\left(\frac{J^{k} \delta^{k}}{J^{k} \delta^{k} \mathfrak{m}}\right)^{i+N k, j+N k} .
$$

5.8. Euler operator. As in [GS, (2.4)] the rings of differential operators $D(\mathfrak{h})$ and $D\left(\mathfrak{h}^{\text {reg }}\right)$ have a graded structure, given by the adjoint action $[\mathbf{E},-]$ of the Euler operator $\mathbf{E}=\sum x_{i} \partial_{i} \in D(\mathfrak{h})$. We will call this the Euler grading and write E-deg for the corresponding degree function; thus $\mathbf{E}$-deg $x_{i}=1$ and $\mathbf{E}$-deg $\partial_{i}=-1$. Since $\mathbf{E} \in D(\mathfrak{h})^{W}, \mathbf{E}$ commutes with $W$ in $D\left(\mathfrak{h}^{\text {reg }}\right) * W$ and so this grading extends to that ring with E-deg $W=0$. By inspection, (3.1.1) implies that the elements $y_{i}$ defined there also have degree -1 and so each $H_{c}$ is also graded under $[\mathbf{E},-]$ and we continue to call this the Euler grading.

It is well-known and easy to check that the $\mathbf{E}$-grading is compatible with the order filtration on $D\left(\mathfrak{h}^{\mathrm{reg}}\right) * W$, in the sense that $\left[\mathbf{E}, \operatorname{ord}^{n} D\left(\mathfrak{h}^{\text {reg }}\right) * W\right] \subseteq \operatorname{ord}^{n} D\left(\mathfrak{h}^{\text {reg }}\right) * W$ for all $n \geq 0$. Thus, we obtain an induced grading, again called the E-grading, on the associated graded object ogr $D\left(\mathfrak{h}^{\text {reg }}\right) * W \cong \mathbb{C}\left[\mathfrak{h}^{\text {reg }} \oplus \mathfrak{h}^{*}\right] * W$ and its subrings. Clearly this is again given by $\mathbf{E}-\operatorname{deg} \mathfrak{h}^{*}=1$ (which we define to mean that $\mathbf{E}-\operatorname{deg}(x)=1$ for every 
$0 \neq x \in \mathfrak{h}^{*}$ ) while $\mathbf{E}$ - $\operatorname{deg} \mathfrak{h}=-1$ and $\mathbf{E}$-deg $W=0$. In other words, the $\mathbf{E}$-grading coincides with the grading induced by the second component of $\mathbb{T}^{2}$ in (5.7); which is the point of defining that action.

5.9. The first conjecture from [BEG2, Section 7] asks whether the isomorphism from Proposition 5.4 can be extended to an appropriate $\mathbb{T}^{2}$-equivariant isomorphism. However the $\mathbb{T}^{2}$-action in [BEG2] is not the one arising from the ord filtration and E-grading; we must replace the ord filtration by the filtration tot defined by the total degree of differential operators. Fortunately, as we next show, it is easy to pass between the two filtrations.

We begin with the formal definitions. The filtration tot is defined on $D\left(\mathfrak{h}^{\text {reg }}\right) * W$ and its subspaces by giving $W$ total degree zero and $\mathfrak{h} \oplus \mathfrak{h}^{*} \backslash\{0\}$ total degree one. As usual, for any subset $M$ of $D\left(\mathfrak{h}^{\text {reg }}\right) * W$, we write $\operatorname{tot}^{r} M$ for the elements $m \in M$ of total degree tot-deg $(m) \leq r$ and denote the associate graded object by $\operatorname{tgr} M=\bigoplus_{r \geq 0} \operatorname{tgr}^{r} M$, where $\operatorname{tgr}^{r} M=\operatorname{tot}^{r} M / \operatorname{tot}^{r-1} M$. It follows from (5.8) that commutation by $\mathbf{E}$ preserves the ord and tot filtrations on $D\left(\mathfrak{h}^{\text {reg }}\right) * W$ and therefore induces a grading on $B_{i j}$ and its associated graded modules. Write $\operatorname{tot}^{r, s} B_{i j}$ (respectively ord ${ }^{r, s} B_{i j}$ ) to denote the elements of $\operatorname{tot}^{r} B_{i j}$ (respectively $\operatorname{ord}^{r} B_{i j}$ ) with E-degree equal to $s$. The E-graded decompositions $\operatorname{tgr}^{r} B_{i j}=\bigoplus_{s \in \mathbb{Z}} \operatorname{tgr}^{r, s} B_{i j}$ and $\operatorname{ogr}^{r} B_{i j}=\bigoplus \operatorname{ogr}^{r, s} B_{i j}$ are defined analogously. By construction, this bigraded structure on $\operatorname{tgr} B_{i j}$ is simply the one coming from the $\mathbb{T}^{2}$-action of (5.7).

Lemma. For each $i \geq j \geq 0$ there is an isomorphism $\operatorname{tgr} B_{i j} \rightarrow \operatorname{ogr} B_{i j}$ which identifies $\operatorname{tg}^{2 r+s, s} B_{i j}$ with $\operatorname{ogr}^{r, s} B_{i j}$. These isomorphisms induce a $\mathbb{Z}$-algebra isomorphism $\operatorname{tgr} B \rightarrow \operatorname{ogr} B$.

Proof. We fix $i \geq j \geq 0$ throughout the proof. By [GS, Lemma 6.10 and Corollary 6.14(1)] the action of $\mathbf{E}$ on $B_{i j}$ is diagonalisable. Therefore

$$
\operatorname{tgr} B_{i j}=\bigoplus_{s \in \mathbb{Z}} \bigoplus_{r \in \mathbb{Z}} \frac{\operatorname{tot}^{r, s} B_{i j}}{\operatorname{tot}^{r-1, s} B_{i j}} \quad \text { and } \quad \operatorname{ogr} B_{i j}=\bigoplus_{s \in \mathbb{Z}} \bigoplus_{r \in \mathbb{Z}} \frac{\operatorname{ord}^{r, s} B_{i j}}{\operatorname{ord}^{r-1, s} B_{i j}} .
$$

If $b \in \operatorname{ord}^{r} B_{i j} \backslash \operatorname{ord}^{r-1} B_{i j}$, set ord-deg $(b)=r$. Then tot-deg $(b)=\mathbf{E}-\operatorname{deg}(b)+2$ ord-deg $(b)$ and so

$$
\begin{aligned}
\operatorname{ord}^{r, s} B_{i j} & =\left\{b \in B_{i j}: \operatorname{ord}-\operatorname{deg}(b) \leq r \text { and } \mathbf{E}-\operatorname{deg}(b)=s\right\} \\
& =\left\{b \in B_{i j}: \operatorname{tot}-\operatorname{deg}(b) \leq 2 r+s \text { and } \mathbf{E}-\operatorname{deg}(b)=s\right\} \\
& =\operatorname{tot}^{2 r+s, s} B_{i j} .
\end{aligned}
$$

This proves the first claim of the lemma. The second claim follows since the map $\operatorname{tgr} B \rightarrow \operatorname{ogr} B$ is induced by the identity map on $B$.

5.10. Assume that $c=1 / n$. By [GS, Proposition 3.16] we can identify

$$
e L_{c+k}(\text { triv }) \cong e H_{c+k} \delta e_{-} \otimes_{U_{c+k-1}} e H_{c+k-1} \delta e \otimes_{U_{c+k-2}} \cdots \otimes_{U_{c+1}} e H_{c+1} \delta e \otimes_{U_{c}} \mathbb{C}
$$

and

$$
L_{c+k} \text { (triv) } \cong H_{c+k} \delta e_{-} \otimes_{U_{c+k-1}} e H_{c+k-1} \delta e \otimes_{U_{c+k-2}} \cdots \otimes_{U_{c+1}} e H_{c+1} \delta e \otimes_{U_{c}} \mathbb{C} .
$$


These modules have an $\mathbf{E}$-gradation and a filtration induced from the tensor product tot-filtration, which induce a bigraded structure on $\operatorname{tgr} L_{c+k}$ (triv) and $\operatorname{tgr} e L_{c+k}$ (triv). Moreover, since the tot filtration is $W$ stable, $\operatorname{tgr} L_{c+k}$ (triv) is then a bigraded $W$-module.

We need a minor extra adjustment to the tot filtration in order to state (and prove) the conjectures from [BEG2]. To be precise, the conjectures are stated in [BEG2] for the tensor product filtration arising from the isomorphism

$$
e L_{c+k}(\text { triv }) \cong e H_{c+k} e_{-} \otimes_{U_{c+k-1}} e H_{c+k-1} e_{-} \otimes_{U_{c+k-2}} \cdots \otimes_{U_{c+1}} e H_{c+1} e_{-} \otimes_{U_{c}} \mathbb{C} .
$$

In other words, one ignores the copy of $\delta$ in each tensor summand of $e L_{c+k}$. We will write the tot filtration arising from (5.10.3) as tot $_{\mathrm{B}}$. Of course, this is only a book-keeping device to account for the powers of $\delta$ and so it follows immediately that

$$
\operatorname{tgr}_{\mathrm{B}}^{r, s} e L_{c+k}(\text { triv })=\operatorname{tgr}^{r+N k, s+N k} e L_{c+k}(\text { triv }) \quad \text { where } \quad N=n(n-1) / 2=\operatorname{deg} \delta .
$$

Using (5.10.2) in place of (5.10.1), the same comments apply to $L_{c+k}$ (triv) and give

$$
\operatorname{tgr}_{\mathrm{B}}^{r, s} L_{c+k}(\text { triv })=\operatorname{tgr}^{r+N k, s+N k} L_{c+k}(\text { triv). }
$$

5.11. We now confirm [BEG2, Conjectures 7.2 and 7.3].

Theorem. Let $c=1 / n$ and $k \in \mathbb{N}$. Give $L_{c}$ (triv) the trivial filtration, and consider the $\mathbb{T}^{2}$-action of (5.7).

(1) Give $e L_{c+k}$ (triv) the tensor product filtration arising from (5.10.1). Then there is a bigraded isomorphism $\operatorname{tgr}_{\mathrm{B}} e L_{c+k}($ triv $) \cong \mathrm{H}^{0}\left(\mathrm{Z}_{\mathrm{n}}, \mathcal{L}^{k}\right)$.

(2) Give $L_{c+k}$ (triv) the tensor product filtration arising from the identity (5.10.2). Then there is a $W$-equivariant, bigraded isomorphism

$$
\operatorname{tgr}_{\mathrm{B}} L_{c+k}(\text { triv }) \cong \mathrm{H}^{0}\left(\mathrm{Z}_{\mathrm{n}}, \mathcal{P} \otimes \mathcal{L}^{k-1}\right) \otimes \operatorname{sign} .
$$

Proof. (1) By Lemma 5.9 and [GS, Lemma 7.2], the tensor product filtration on $B_{i j}$ induced from the tot filtration on the $B_{k+1, k}$ coincides with the tot filtration on $B_{i j}$. So we need not distinguish between them. As in the proof of Proposition 5.4, identify $L=e L_{c}$ (triv) $=\mathbb{C}=U_{c} / \widehat{\mathfrak{m}}$ and give $L$ the trivial filtration. Then, for any $k \geq 0$, the induced tensor product filtration on $L(k) \cong e L_{c+k}($ triv $)$ is equal to the one coming from the tensor product filtration on the right hand side of (5.10.1). By Lemma 5.3 there is a surjection $\eta^{k}: \operatorname{tgr}\left(B_{k 0}\right) / \operatorname{tgr}\left(B_{k 0}\right) \operatorname{tgr}(\widehat{\mathfrak{m}}) \rightarrow \operatorname{gr} L(k)$. This is clearly a graded morphism under both the tot and $\mathbf{E}$-gradings.

Now tgr $\widehat{\mathfrak{m}}=\mathfrak{m}=\mathbb{C}\left[\mathfrak{h} \oplus \mathfrak{h}^{*}\right]_{+}^{W}$. Thus (3.9.1) and Lemma 5.9 give bigraded isomorphisms

$$
\left(\frac{\operatorname{tgr} B_{k 0}}{\left(\operatorname{tgr} B_{k 0}\right)(\operatorname{tgr} \widehat{\mathfrak{m}})}\right)^{r+N k, s+N k} \cong\left(\frac{A^{k} \delta^{k}}{A^{k} \delta^{k} \mathfrak{m}}\right)^{r+N k, s+N k}
$$

for all $r, s \in \mathbb{Z}$. By (5.7.1), this final term is isomorphic to $\mathrm{H}^{0}\left(\mathrm{Z}_{\mathrm{n}}, \mathcal{L}^{k}\right)^{r, s}$. Thus, (5.10.4) and (5.11.1) combine to show that, for each $r$ and $s$, the map $\eta^{k}$ restricts to a surjection $\eta_{r, s}: \mathrm{H}^{0}\left(\mathrm{Z}_{\mathrm{n}}, \mathcal{L}^{k}\right)^{r, s} \rightarrow \operatorname{tgr}_{\mathrm{B}}^{r, s} e L_{c+k}($ triv $)$. 
Finally, by Lemma 3.15 and [BEG2, Theorem 1.14], we know that $\operatorname{dim}_{\mathbb{C}} \mathrm{H}^{0}\left(\mathrm{Z}_{\mathrm{n}}, \mathcal{L}^{k}\right)=\operatorname{dim} e L_{c+k}($ triv $)<\infty$. Therefore, each $\eta_{r, s}$ must be an isomorphism and we are done.

(2) In this case, the filtration on each $L_{c+k}$ (triv) is the one coming from the tensor product filtration on the right hand side of (5.10.2). However, by [GS, Lemma 7.2] this is the same filtration as that coming from the tensor product filtration on $L_{c+k} \cong M \otimes_{U_{c}} \mathbb{C}$, where $M=H_{c+k} \delta e B_{k-10}$. Thus, by (5.10.5),

$$
\operatorname{tot}_{B}^{r, s} L_{c+k}=\operatorname{tot}^{r+N k, s+N k} M \otimes \mathbb{C} .
$$

By Lemma 5.3 there is a surjection $\chi: \operatorname{tgr} M /(\operatorname{tgr} M)(\operatorname{tgr} \widetilde{\mathfrak{m}}) \rightarrow \operatorname{tgr}\left(L_{c+k}\right)$, which is bigraded under the tot and E gradings. By [GS, Proposition B.1],

$$
\left(\frac{\operatorname{tgr} M}{\operatorname{tgr} M \operatorname{tgr} \widehat{\mathfrak{m}}}\right)^{r+N k, s+N k} \cong\left(\frac{J^{k-1} \delta^{k}}{J^{k-1} \delta^{k} \mathfrak{m}}\right)^{r+N k, s+N k} \cong \operatorname{sign} \otimes\left(\frac{J^{k-1} \delta^{k-1}}{J^{k-1} \delta^{k-1} \mathfrak{m}}\right)^{r+N(k-1), s+N(k-1)}
$$

for all $r, s \in \mathbb{Z}$. By (5.7.1), again, the right hand side of (5.11.3) is isomorphic to $\mathrm{H}^{0}\left(\mathrm{Z}_{\mathrm{n}}, \mathcal{P} \otimes \mathcal{L}^{\mathrm{k}-1}\right)^{\mathrm{r}, \mathrm{s}} \otimes$ sign. By construction and Lemma 3.12, each of these identifications is $W$-equivariant. Therefore, (5.11.3) and (5.11.2) combine to give a $W$-equivariant surjection

$$
\chi^{r, s}: \mathrm{H}^{0}\left(\mathrm{Z}_{\mathrm{n}}, \mathcal{P} \otimes \mathcal{L}^{k-1}\right)^{r, s} \otimes \operatorname{sign} \rightarrow \operatorname{tgr}_{B}^{r, s} L_{c+k}
$$

for each $r$ and $s$. By [BEG2, Theorem 1.11] and Lemma 3.15 we know that $\operatorname{dim} \operatorname{tgr}_{B} L_{c+k}=(k n+1)^{n-1}=$ $\operatorname{dim} \mathrm{H}^{0}\left(\mathrm{Z}_{\mathrm{n}}, \mathcal{P} \otimes \mathcal{L}^{k-1}\right)$ for all $k \geq 1$. Hence $\chi^{r, s}$ is an isomorphism, as required.

5.12. Using (3.15.2) and (3.16.1) one can explicitly write down the two variable Poincaré series for $L_{c+k}$ (triv) and $e L_{c+k}$ (triv) arising from this theorem. Of course, by (5.7), those formulæ need to be slightly modified to apply here but, for example, it follows from (3.16.1) that under the grading of this theorem, $e L_{c+k}$ (triv) has the two-variable Poincaré series $p\left(e L_{c+k}(\right.$ triv $\left.), s, t\right)=C_{n}^{(k)}\left(s t, s t^{-1}\right)$.

\section{Characteristic CyCles}

6.1. In this section we expand on the results from Sections 4 and 5 in order to understand the characteristic varieties Char $M \subseteq \operatorname{Hilb}(\mathrm{n})$ and the characteristic cycles $\mathbf{C h} M$ for category $\mathcal{O}$-modules $M$ (the reader should recall the notation and conventions from Section 2 concerning these objects). Even for a standard module $\Delta=\Delta_{c}(\mu)$, the answer is quite subtle and in marked contrast to the usual associated variety $\operatorname{Char}_{0} \Delta \subseteq \mathfrak{h} \oplus \mathfrak{h}^{*} / W$. Indeed, $\operatorname{Char}_{0} \Delta$ is clearly independent of $\mu$ whereas Char $\Delta$ varies with and even determines $\mu$. The precise result is given in Theorem 6.7 which also gives an explicit formula for the characteristic cycle $\mathbf{C h} \Delta$. Our results are less complete for other objects of $\mathcal{O}_{c}$, but they are sufficient to show in Corollary 6.10 that $K\left(\mathcal{O}_{c}\right)$ is isomorphic to the top degree Borel-Moore homology group $\mathrm{H}_{2 n-2}(Z, \mathbb{C})$ for the variety $Z=\tau^{-1}(\mathfrak{h} / W)$.

As usual we will assume that $c$ satisfies Hypothesis 3.4 throughout the section. 
6.2. Before proceeding, we will need a minor variant of $\left[\mathrm{GS}\right.$, Corollary 4.13]. Let $\mathbb{C}[\mathfrak{h}]^{\operatorname{co} W}=\mathbb{C}[\mathfrak{h}] / \mathbb{C}[\mathfrak{h}]_{+}^{W} \mathbb{C}[\mathfrak{h}]$ denote the algebra of coinvariants. For $\mu \in \operatorname{Irrep}(W)$ define the fake degree of $\mu$ to be the polynomial $f_{\mu}(v)=\sum_{i \geq 0}\left[\mathbb{C}[\mathfrak{h}]_{i}^{\operatorname{co} W}: \mu\right] v^{i}$.

Recall from (5.8) that $\mathbb{C}\left[\mathfrak{h} \oplus \mathfrak{h}^{*}\right]$ has a natural $\mathbb{Z}$-grading called the $\mathbf{E}$ grading. Given an E-graded module $M=\bigoplus M_{i}$, we will write the corresponding Poincaré series as $p(M, v)=\sum v^{i} \operatorname{dim}_{\mathbb{C}} M_{i}$.

Lemma. Set $N=n(n-1) / 2$ and write $K=\mathfrak{h} \mathbb{C}\left[\mathfrak{h} \oplus \mathfrak{h}^{*}\right] \subset \mathbb{C}\left[\mathfrak{h} \oplus \mathfrak{h}^{*}\right]$ for the ideal generated by the elements of $\mathfrak{h}$. Then $J^{k} \delta^{k} / J^{k} \delta^{k} K$ is $\mathbf{E}$-graded for any $k \geq 1$ and has Poincaré series

$$
p\left(J^{k} \delta^{k} / J^{k} \delta^{k} K, v\right)=v^{N k} \frac{\sum_{\mu} f_{\mu}(1) f_{\mu}(v) v^{k\left(n(\mu)-n\left(\mu^{t}\right)\right)}}{\prod_{i=2}^{n}\left(1-v^{i}\right)} .
$$

Proof. As in [GS, (4.13)], it is routine to check that the given modules are E-graded.

Let $C=\mathbb{C}\left[\mathfrak{h}^{*}\right]_{+}^{W}$. Since $J^{1}$ is symmetric in $\mathfrak{h}$ and $\mathfrak{h}^{*}$, it follows from $[$ GS, Corollary 4.13$]$ that $J^{k} / J^{k} C$ has Poincaré series

$$
p\left(J^{k} / J^{k} C, v\right)=\frac{\sum_{\mu} f_{\mu}(1) f_{\mu}(v) v^{k\left(n(\mu)-n\left(\mu^{t}\right)\right)}[n]_{v^{-1}} !}{\prod_{i=2}^{n}\left(1-v^{i}\right)}, \quad \text { where }[n]_{v} !=\frac{\prod_{i=1}^{n}\left(1-v^{i}\right)}{(1-v)^{n}} .
$$

The derivation of this formula in [GS] was obtained by noting that the fundamental invariants, which provide the generators of $C$, form an r-sequence in each $J^{k}$. This allows one to obtain the Poincaré series for $J^{k} / J^{k} C$ from the Poincaré series for $J^{k}$ given by [GS, Corollary 4.11]. By [GS, Lemma 4.4(2)], the natural generators of $K$ also form an r-sequence in $J^{k}$ and so the same argument can be used to find the Poincaré series of $J^{k} / J^{k} K$.

In more detail, the generators of $K$ all have degree -1 while the fundamental invariants have degrees $-2 \geq-r \geq-n$ and $\delta^{k}$ has degree $N k$. In the notation of [GS, (4.11)], the formula [GS, (4.13.3)] gives

$$
p\left(J^{k} / J^{k} C, v\right)=\left(\prod_{i=2}^{n}\left(1-t^{i}\right) p\left(J^{k}, s, t\right)\right)_{s=v, t=v^{-1}} .
$$

Using the same argument one obtains:

$$
p\left(J^{k} \delta^{k} / J^{k} \delta^{k} K, v\right)=v^{N k}\left((1-t)^{n-1} p\left(J^{k}, s, t\right)\right)_{s=v, t=v^{-1}} .
$$

Comparing these two formulæ gives

$$
p\left(J^{k} \delta^{k} / J^{k} \delta^{k} K, v\right)=\frac{v^{N k}\left(1-v^{-1}\right)^{(n-1)}}{\prod_{i=2}^{n}\left(1-v^{-i}\right)} p\left(J^{k} / J^{k} C, v\right)=\frac{v^{N k}}{[n]_{v^{-1}} !} p\left(J^{k} / J^{k} C, v\right) .
$$

Combined with (6.2.2) this implies (6.2.1).

6.3. The next few results give partial information on the characteristic cycle $\mathbf{C h} \Delta$ for a standard module $\Delta$ that will be used in the complete description of $\mathbf{C h} \Delta$ in Theorem 6.7.

For a $W$-module $V$ and $\mu \in \operatorname{Irrep}(W)$ set $V_{\mu}=(V \otimes \mu)^{W}$. By construction, the fibres of the Procesi bundle $\mathcal{P}$ carry the regular $W$-representation and so $\mathcal{P}_{\mu}$ is a vector bundle of rank $\operatorname{dim} \mu$. Write $K=\mathfrak{h} \mathbb{C}\left[\mathfrak{h} \oplus \mathfrak{h}^{*}\right]$ as 
in (6.2). It follows from (3.7) that $\mathbb{C}\left[\mathfrak{h} \oplus \mathfrak{h}^{*}\right] \cong \mathcal{O}\left(X_{n}\right)=\mathrm{H}^{0}(\operatorname{Hilb}(\mathrm{n}), \mathcal{P})$. Thus $\mathbb{C}\left[\mathfrak{h} \oplus \mathfrak{h}^{*}\right]$ acts on $\mathcal{P}$ and so $\mathcal{P} K$ and $(\mathcal{P} K)_{\mu}$ are naturally defined for any $\mu \in \operatorname{Irrep}(W)$.

Let $c \geq 0$. We give $\Delta_{c}(\mu)$ the induced filtration $\Gamma^{t}\left(\Delta_{c}(\mu)\right)=\operatorname{ord}^{t} H_{c} \cdot(1 \otimes \mu)$. Since $\Delta_{c}(\mu) \cong \mathbb{C}[\mathfrak{h}] \otimes \mu$, this is the same as the trivial filtration $\Gamma^{t}\left(\Delta_{c}(\mu)\right)=\Delta_{c}(\mu)$ for all $t$, but the present formulation will be more convenient in the sequel.

Lemma. For each $\mu \in \operatorname{Irrep}(W)$ there is an isomorphism $\widehat{\Phi}_{\Gamma}\left(\Delta_{c}(\mu)\right) \cong \mathcal{P}_{\mu} /(\mathcal{P} K)_{\mu}$ of sheaves on $\operatorname{Hilb}(\mathrm{n})$.

Proof. Let $X=H_{c} \otimes_{\mathbb{C}\left[\mathfrak{h}^{*}\right]} \mathbb{C}$, filtered by $\Gamma^{t}(X)=\operatorname{ord}^{t} H_{c} \cdot(1 \otimes \mathbb{C})$, and set

$$
X(k)=S_{c+k-1} \circ \cdots \circ S_{c}(e X) \cong e H_{c}(k) \otimes_{\mathbb{C}\left[\mathfrak{h}^{*}\right]} \mathbb{C},=B_{k 0} \otimes_{U_{c}} e H_{c} \otimes_{\mathbb{C}\left[\mathfrak{h}^{*}\right]} \mathbb{C} \quad \text { for } k \in \mathbb{N} .
$$

The filtration $\Gamma$ on $X$ induces the tensor product filtration on $X(k)$, which we also call $\Gamma$. By [GS, Lemma 7.2], the tensor product and ord filtrations on $B_{k 0} \otimes_{U_{c}} e H_{c}$ coincide and so $\Gamma^{t} X(k)=\left(\operatorname{ord}^{t} e H_{c}(k)\right) \cdot(1 \otimes \mathbb{C})$ for all $t$ and $k$. Thus, by Theorem 4.5, there is a surjective mapping $p^{\prime}: J^{k} \delta^{k} \rightarrow \operatorname{gr}_{\Gamma} X(k)$. Moreover, since $\mathbb{C}\left[\mathfrak{h}^{*}\right]$ acts trivially on $1 \otimes 1 \in X$, this factors through a surjection

$$
p: \frac{J^{k} \delta^{k}}{J^{k} \delta^{k} K} \longrightarrow \operatorname{gr}_{\Gamma} X(k) .
$$

Since the $\mathbf{E}$-gradation commutes with the ord-filtration and the isomorphism of Theorem 4.5 is $\mathbf{E}$-graded, the map $p$ is E-graded. Comparing [GS, Corollary 6.14(2)] with Lemma 6.2 shows that $X(k)$ (which equals $N(k)$ in the notation of [GS]) and $J^{k} \delta^{k} / J^{k} \delta^{k} K$ have the same Poincaré series under the E-grading. Since $p$ is surjective on each (finite dimensional) component, it is therefore an isomorphism. Equivalently, $p$ induces an isomorphism $\operatorname{gr}_{\Gamma} \tilde{X} \cong \bigoplus_{k \geq 0} J^{k} \delta^{k} / J^{k} \delta^{k} K$.

There is a natural action of $W$ on $X(k)$ given by $w \cdot(r \otimes 1)=r w^{-1} \otimes 1$ for $w \in W$ and $r \in H_{c}(k)$, with the induced action on $\operatorname{gr}_{\Lambda} X(k)$. We next show that $p$ is $W$-equivariant under this action. For $r \in H_{c}(k)$, let $\bar{r}$ denote the image of the principal symbol $\sigma(r)$ in $J^{k} \delta^{k} / J^{k} \delta^{k} K$. The definition of multiplication in $D\left(\mathfrak{h}^{\text {reg }}\right) * W$ from (3.1) says that $\omega r \omega^{-1}=\omega(r)$, for $r \in \mathbb{C}[\mathfrak{h}] \mathbb{C}\left[\mathfrak{h}^{*}\right] \subseteq H_{c+k}$ and $\omega \in W$. By [GS, Lemma 6.11(1)] and (3.2.1), $e H_{c}(k) \subseteq e H_{c+k}=e \mathbb{C}[\mathfrak{h}] \mathbb{C}\left[\mathfrak{h}^{*}\right]$ and so the construction of $p^{\prime}$ implies that, under the natural action of $W$ on $J^{k} \delta^{k}$,

$$
p(\omega(\bar{r})) \equiv e \omega(\sigma(r)) \otimes 1 \equiv e \omega \sigma(r) \omega^{-1} \otimes 1 \equiv e \sigma(r) \omega^{-1} \otimes 1 \equiv \omega(p(\bar{r})) \quad \bmod J^{k} \delta^{k} K
$$

for all such $r$ and $\omega$. Thus $p$ and (by construction) the functors $S_{c+j}$ are indeed $W$-equivariant.

Next, give $H_{c} \otimes_{\mathbb{C}\left[\mathfrak{h}^{*}\right] * W} \mathbb{C} W$ the $W$-action analogous to that on $X$ so the isomorphism $X \cong H_{c} \otimes_{\mathbb{C}[\mathfrak{h} *] * W} \mathbb{C W}$ is $W$-equivariant. Then

$$
X_{\mu} \cong\left[H_{c} \otimes_{\mathbb{C}\left[\mathfrak{h}^{*}\right] * W} \mathbb{C} W\right]_{\mu}=H_{c} \otimes_{\mathbb{C}\left[\mathfrak{h}^{*}\right] * W}(\mathbb{C} W)_{\mu}=H_{c} \otimes_{\mathbb{C}\left[\mathfrak{h}^{*}\right] * W} \mu=\Delta_{c}(\mu)
$$

Since $p$ and the $S_{j}$ are $W$-equivariant, (6.3.1) induces an isomorphism

$$
\left(J^{k} \delta^{k} / J^{k} \delta^{k} K\right)_{\mu} \cong\left(\operatorname{gr}_{\Lambda} X(k)\right)_{\mu}=\operatorname{gr}_{\Lambda}\left(X_{\mu}(k)\right)=\operatorname{gr}_{\Lambda} e \Delta_{c}(\mu)(k) .
$$


Thus (6.3.2) induces an isomorphism of graded $A$-modules

$$
\widetilde{e \Delta_{c}(\mu)} \cong \bigoplus_{k \geq 0}\left(\frac{J^{k} \delta^{k}}{J^{k} \delta^{k} K}\right)_{\mu}
$$

Finally, we need to translate this equation to one involving sheaves. Consider the short exact sequence

$$
0 \longrightarrow \bigoplus_{k \geq 0} J^{k} \delta^{k} K \longrightarrow \bigoplus_{k \geq 0} J^{k} \delta^{k} \longrightarrow \bigoplus_{k \geq 0} \frac{J^{k} \delta^{k}}{J^{k} \delta^{k} K} \longrightarrow 0
$$

of $A$-modules. By (3.7), the image of this sequence in $A$-qgr $\cong$ Coh Hilb(n) is isomorphic to the short exact sequence of sheaves $0 \rightarrow \mathcal{P} K \rightarrow \mathcal{P} \rightarrow \mathcal{P} / \mathcal{P} K \rightarrow 0$. By (3.8.1) this isomorphism is $W$-equivariant and so the module $\bigoplus_{k \geq 0}\left(J^{k} \delta^{k}\right)_{\mu} \in A$-grmod has image $\mathcal{P}_{\mu} \in \operatorname{Coh} \operatorname{Hilb}(\mathrm{n})$. Combining these facts shows that the image of (6.3.3) in Coh Hilb(n) is precisely $\mathcal{P}_{\mu} /(\mathcal{P} K)_{\mu}$.

6.4. To describe the characteristic cycles of objects from $\mathcal{O}_{c}$ we need to recall a set of Lagrangian subvarieties which were introduced by Grojnowski in [Gr, Section 3] (see also [Na, Sections 7.2 and 9.2]).

Recall from (3.6) that we identify $\mathbb{C}^{2 n} / W$ with $S^{n} \mathbb{C}^{2}$, write $\widehat{\tau}:$ Hilb $^{n} \mathbb{C}^{2} \rightarrow \mathbb{C}^{2 n} / W$ and identify $\mathfrak{h}$ with a subspace of $\mathbb{C}^{n} \times \mathbf{0} \subset \mathbb{C}^{2 n}$. Set $\widetilde{C}=\widehat{\tau}^{-1}\left(\mathbb{C}^{n} \times \mathbf{0}\right)$; equivalently $\widetilde{C}=\left\{I \in \operatorname{Hilb}^{\mathrm{n}} \mathbb{C}^{2}: \operatorname{Supp} \mathbb{C}[x, y] / I \subseteq S^{n} C\right\}$ where $C=\{(z, 0): z \in \mathbb{C}\} \subseteq \mathbb{C}^{2}$. By [Gr, Proposition 3], $\widetilde{C}$ is a pure variety of dimension $n$. To describe its irreducible components, we use the stratification $S^{n} \mathbb{C}^{2}=\bigsqcup_{\lambda \vdash n} S_{\lambda} \mathbb{C}^{2}$ where, for a partition $\lambda=\left(\lambda_{1}, \ldots, \lambda_{r}\right)$ with exactly $r$ non-zero parts,

$$
S_{\lambda} \mathbb{C}^{2}=\left\{\sum_{i=1}^{r} \lambda_{i}\left[x_{i}\right]: x_{1}, \ldots, x_{r} \in \mathbb{C}^{2} \text { are distinct }\right\} .
$$

By [Gr, Proposition 3] or [Na, p.111] the irreducible components of $\widetilde{C}$ are the closures $\bar{C}_{\lambda}$ of the subvarieties

$$
\widetilde{C}_{\lambda}=\widetilde{C} \cap\left\{I \in \operatorname{Hilb}^{\mathrm{n}} \mathbb{C}^{2}: \operatorname{Supp} \mathbb{C}[x, y] / I \in S_{\lambda} \mathbb{C}^{2}\right\}
$$

Moreover, $\operatorname{dim} \bar{C}_{\lambda}=n$ for all $n$.

Set $Z=\tau^{-1}(\mathfrak{h} / W)$ which, by the last paragraph, is equal to $\widetilde{C} \cap \operatorname{Hilb}(\mathrm{n})$. We will occasionally write $Z=Z(n)$ when we need to specify $n$. In order to identify the components of $Z$, we recall from [GS, Proof of Corollary 4.10] that the identification $\mathbb{C}\left[\mathbb{C}^{2 n}\right]^{W}=\mathbb{C}\left[\mathfrak{h} \oplus \mathfrak{h}^{*}\right]^{W}\left[\mathbf{z}, \mathbf{z}^{*}\right]$ induces a factorisation Hilb $\mathbb{C}^{2}=$ $\operatorname{Hilb}(\mathrm{n}) \times \mathbb{C}^{2}$, where $\mathbb{C}^{2}=\operatorname{Spec} \mathbb{C}\left[\mathbf{z}, \mathbf{z}^{*}\right]$. It follows that $\widetilde{C}=Z \times \mathbb{C}$ and so we have proved the following result.

Lemma. The variety $Z$ is pure of dimension $n-1$ with irreducible components

$$
Z_{\lambda}=\overline{\operatorname{Hilb}(\mathrm{n}) \cap \widetilde{C}_{\lambda}} \quad \text { for } \lambda \in \operatorname{Irrep}(W) .
$$

In this notation, the punctual Hilbert scheme $Z_{\mathrm{n}}$ equals $Z_{(n)}$. 
6.5. Lemma. If $\mu \in \operatorname{Irrep}(W)$ then $\operatorname{Char}\left(\Delta_{c}(\mu)\right)$ is a union of subvarieties $Z_{\lambda}$, for some $\lambda \in \operatorname{Irrep}(W)$. In particular, $\operatorname{Char}\left(\Delta_{c}(\mu)\right)$ is equidimensional.

Proof. By Lemma 6.3, we need to calculate the support variety of the sheaf $\mathcal{P}_{\mu} /(\mathcal{P} K)_{\mu}$. By (6.3.4),

$$
\tau(\operatorname{Supp} \mathcal{P} / \mathcal{P} K)=\mathcal{V}\left(K \cap \mathbb{C}\left[\mathfrak{h} \oplus \mathfrak{h}^{*}\right]^{W}\right)=\mathfrak{h} / W
$$

as a subvariety of $\mathfrak{h} \oplus \mathfrak{h}^{*} / W$. Therefore, by the definition of $Z$, we have $\operatorname{Supp} \mathcal{P}_{\mu} /(\mathcal{P} K)_{\mu} \subseteq Z$.

Let $\left\{y_{1}, \ldots, y_{n-1}\right\}$ be a basis of $\mathfrak{h}$. Arguing as in [GS, (4.9.2)] we see that $y_{1}, \ldots, y_{n-1}$ is a regular sequence in $S=\mathbb{C}\left[\mathfrak{h} \oplus \mathfrak{h}^{*}\right]\left[t J^{1} \delta\right]$, the Rees ring of $X_{n}$, and so we have an $W$-equivariant Koszul resolution

$$
0 \rightarrow \mathcal{O}_{X_{n}} \otimes \bigwedge^{n-1} \mathfrak{h}^{*} \rightarrow \cdots \rightarrow \mathcal{O}_{X_{n}} \otimes \bigwedge^{i} \mathfrak{h}^{*} \rightarrow \cdots \rightarrow \mathcal{O}_{X_{n}} \rightarrow \frac{\mathcal{O}_{X_{n}}}{\mathcal{O}_{X_{n}} K} \rightarrow 0
$$

Tensoring this by $\mu$, applying $\rho_{*}$ and taking $W$-fixed points gives an exact sequence

$$
0 \rightarrow\left(\mathcal{P} \otimes \bigwedge^{n-1} \mathfrak{h}^{*} \otimes \mu\right)^{W} \rightarrow \cdots \rightarrow\left(\mathcal{P} \otimes \bigwedge^{i} \mathfrak{h}^{*} \otimes \mu\right)^{W} \rightarrow \cdots \rightarrow(\mathcal{P} \otimes \mu)^{W} \rightarrow \frac{\mathcal{P}_{\mu}}{(\mathcal{P} K)_{\mu}} \rightarrow 0
$$

As this resolution has length $n-1$, the new intersection theorem [BKR, Corollary 5.2] implies that the irreducible components of the support of the cohomology sheaves have codimension at most $n-1$ in Hilb(n). In other words, the irreducible components of $\operatorname{Supp} \mathcal{P}_{\mu} /(\mathcal{P} K)_{\mu}$ have dimension at least $n-1$. Since $\operatorname{Supp} \mathcal{P}_{\mu} /(\mathcal{P} K)_{\mu} \subseteq Z$, the result therefore follows from Lemma 6.4.

6.6. Lemma. The multiplicity mult $_{Z_{(n)}}(\mathcal{P} / \mathcal{P} K)$ equals 1 .

Proof. Let $\mathcal{P}_{1}$ denote the Procesi bundle on $\mathrm{Hilb}^{\mathrm{n}} \mathbb{C}^{2}$, as in the proof of Lemma 3.12. Then $\mathcal{P} / \mathcal{P} K=$ $\mathcal{P}_{1} /\left(\mathcal{P}_{1} K+\mathcal{P}_{1}\left(\mathbf{z}, \mathbf{z}^{*}\right)\right)$ under the embedding $\mathbb{C}\left[\mathfrak{h} \oplus \mathfrak{h}^{*}\right] \hookrightarrow \mathbb{C}\left[\mathfrak{h} \oplus \mathfrak{h}^{*}\right]\left[\mathbf{z}, \mathbf{z}^{*}\right]=\mathbb{C}\left[\mathbb{C}^{2 n}\right]$ of (3.6).

For a partition $\lambda$ of $n$, recall that the monomial ideal $I_{\lambda}$ from (3.15.1) is a point of $Z_{n}$. We claim, as is well-known, that $I_{\lambda}$ is also a point of $Z_{\lambda}$. To see this, suppose that $\sum_{i=1}^{r} \lambda_{i}\left[q_{i}\right] \in S_{\lambda} \mathbb{C}^{2} \cap \mathfrak{h}$. Then the ideal

$$
I(\lambda, \mathbf{q})=\bigcap_{i=1}^{r}\left(y^{\lambda_{i}} \mathbb{C}[x, y]+\left(x-q_{i}\right) \mathbb{C}[x, y]\right)
$$

clearly belongs to $Z_{\lambda}$. Since $S_{\lambda} \mathbb{C}^{2}$ is stable under the $\mathbb{T}^{2}$ action of (3.13), so is $Z_{\lambda}$. Thus, $I_{\lambda}=\lim _{t \rightarrow 0} I(\lambda, t \mathbf{q})$ is also in $Z_{\lambda}$.

Since $I_{\lambda}$ is killed by $\mathbf{z}$, the geometric fibres of $\mathcal{P} / \mathcal{P} K$ and $\mathcal{P}_{1} /\left(\mathcal{P}_{1} K+\mathcal{P}_{1} \mathbf{z}^{*}\right)$ at $I_{\lambda}$ are equal. Let $\mathbb{T}^{2}$ act on $\mathbb{C}^{2}$ as in (3.13), and use the conventions of (3.13) for the corresponding bigraded decomposition $\mathcal{P}_{1}\left(I_{\lambda}\right)=$ $\sum_{i j} \mathcal{P}_{1}\left(I_{\lambda}\right)^{i j}$. These bigraded components are $W$-modules; indeed, by [Ha3, (46) and Proposition 3.4] (which is proved in [Ha2, Section 3.9]), the bigraded $W$-equivariant structure of $\mathcal{P}_{1}\left(I_{\lambda}\right)$ is given by $\left[\mathcal{P}_{1}\left(I_{\lambda}\right)\right]=$ $\sum_{\mu} s^{n(\lambda)} K_{\mu \lambda}\left(t, s^{-1}\right)[\mu]$. Factoring $\mathcal{P}_{1}$ by $\mathcal{P}_{1} K+\mathcal{P}_{1} \mathbf{z}^{*}$ kills precisely the elements with positive $\mathfrak{h}$-degree, and so the bigraded $W$-structure of $\mathcal{P}_{1}\left(I_{\mu}\right) /\left(\mathcal{P}_{1} K+\mathcal{P}_{1} \mathbf{z}^{*}\right)\left(I_{\mu}\right)$ is given by $\sum_{\lambda} s^{n(\lambda)} K_{\mu \lambda}\left(0, s^{-1}\right)[\mu]$. Specialising $s$ to 1 and using (3.11) shows that the $W$-equivariant structure of these fibres is given by

$$
\frac{\mathcal{P}}{\mathcal{P} K}\left(I_{\lambda}\right)=\frac{\mathcal{P}_{1}}{\left(\mathcal{P}_{1} K+\mathcal{P}_{1} \mathbf{z}^{*}\right)}\left(I_{\lambda}\right)=\sum_{\mu} K_{\mu \lambda}[\mu]
$$


In particular $(3.11)$ implies that $\operatorname{dim}(\mathcal{P} / \mathcal{P} K)\left(I_{(n)}\right)=1$. As the dimension of the geometric fibre at $I_{(n)}$ is an upper bound for the multiplicity of $\mathcal{P} / \mathcal{P} K$ along $Z_{(n)}$, this implies that mult $_{Z_{(n)}} \mathcal{P} / \mathcal{P} K \leq 1$.

On the other hand, by construction, $\mathcal{P}_{(n)}=\mathcal{O}_{\mathrm{Hilb}(\mathrm{n})}$ and so there is an inclusion

$$
\mathcal{O}_{Z_{(n)}}=\mathcal{O}_{\operatorname{Hilb}(\mathrm{n})} /\left(K \cap \mathcal{O}_{\operatorname{Hilb}(\mathrm{n})}\right) \subseteq \mathcal{P} / \mathcal{P} K
$$

Since $Z_{(n)}$ is an irreducible component of $Z$ it follows that $1=\operatorname{mult}_{Z_{(n)}} \mathcal{O}_{Z} \leq \operatorname{mult}_{Z_{(n)}} \mathcal{P} / \mathcal{P} K$.

6.7. We can now describe the characteristic cycle of any standard module in terms of the varieties $Z_{\lambda}$ and the Kostka numbers $K_{\mu \lambda}$ from (3.11).

Theorem. Let $\Delta_{c}(\mu)$ be the standard $H_{c}$-module corresponding to $\mu \in \operatorname{Irrep}(W)$. Then

$$
\mathbf{C h}\left(\Delta_{c}(\mu)\right)=\sum_{\lambda} K_{\mu \lambda}\left[Z_{\lambda}\right]
$$

Remarks. (1) By (3.11), $K_{\mu \lambda}=0$ unless $\mu \geq \lambda$ and $K_{\mu \mu}=1$. Combined with the theorem, this therefore shows that $\mu$ is determined by Char $\Delta_{c}(\mu)$.

(2) Very recently, Gan and Ginzburg [GG] have used $\mathcal{D}$-modules to give another way of defining the characteristic cycle of a category $\mathcal{O}_{c}$-module as a subscheme of Hilb(n). It would be interesting to know the relationship between their cycle and ours.

Proof. By Lemma 6.5 it remains to determine the multiplicity of $\mathcal{P}_{\mu} /(\mathcal{P} K)_{\mu}$ along each $Z_{\lambda}$. We will do this by reducing to the case of Lemma 6.6.

We first need to introduce some notation. Let $m \in \mathbb{N}$ and write $\widehat{\tau}_{m}: \operatorname{Hilb}^{m} \mathbb{C}^{2} \rightarrow \mathbb{C}^{2 m} / \mathfrak{S}_{m}$ for the crepant resolution. The Procesi bundles for $\operatorname{Hilb}(m)$ and $\operatorname{Hilb}^{m} \mathbb{C}^{2}$ will be written as $\mathcal{P}(m)$ and $\mathcal{P}_{1}(m)$ respectively, whilst the analogue of $K$ will be written $K(m)$. For $\alpha \in \mathbb{C}$, set

$$
\overline{\mathcal{P}}_{1, \alpha}(m)=\frac{\mathcal{P}_{1}(m)}{\mathcal{P}_{1}(m) K(m)+\mathcal{P}_{1}(m)\left(\mathbf{z}-\alpha, \mathbf{z}^{*}\right)} \quad \text { and } \quad \quad \overline{\mathcal{P}}(m)=\overline{\mathcal{P}}_{1,0}(m)=\frac{\mathcal{P}(m)}{\mathcal{P}(m) K(m)} .
$$

As in [Ha2, Definition 3.2.4] or [GS, (4.2)], the isospectral Hilbert scheme $\mathbb{X}_{m}$ (which is the $\mathbb{C}^{2 m}$ analogue of $\left.X_{n}\right)$ is described as a set by

$$
\mathbb{X}_{m}=\left\{\left(I, p_{1}, \ldots, p_{m}\right): I \in \operatorname{Hilb}^{m} \mathbb{C}^{2}, p_{1}, \ldots, p_{m} \in \mathbb{C}^{2}, \operatorname{Supp} I=\sum\left[p_{i}\right]\right\} .
$$

We have mult $_{Z_{\lambda}} \overline{\mathcal{P}}(n)=$ mult $_{Z_{\lambda}} \overline{\mathcal{P}}_{1,0}(n)$. If $I$ is a generic point of $Z_{\lambda}$ then mult $Z_{\lambda} \overline{\mathcal{P}}_{1,0}(n)=$ mult $_{I} \overline{\mathcal{P}}_{1,0}(n)$, the multiplicity of $\overline{\mathcal{P}}_{1,0}(n)$ at $I$ in $\operatorname{Hilb}^{\mathrm{n}} \mathbb{C}^{2}$. Therefore

$$
\operatorname{mult}_{Z_{\lambda}} \overline{\mathcal{P}}(n)=\operatorname{mult}_{I} \overline{\mathcal{P}}_{1,0}(n)
$$

Write Supp $I=\sum\left[p_{i}\right]=\lambda_{1}\left[q_{1}\right]+\cdots \lambda_{r}\left[q_{r}\right]$ for distinct $q_{1}, \ldots, q_{r} \in C=\mathbb{C} \times\{0\}$. By [Ha2, Lemma 3.3.1] and induction there is a neighbourhood of $\left(I, p_{1}, \ldots, p_{n}\right) \in \mathbb{X}_{n}$ that is isomorphic to a neighbourhood of the point

$$
\left(\left(I_{1}, q_{1}, \ldots, q_{1}\right),\left(I_{2}, q_{2}, \ldots, q_{2}\right), \ldots,\left(I_{r}, q_{r}, \ldots, q_{r}\right)\right) \in \mathbb{X}_{\lambda_{1}} \times \mathbb{X}_{\lambda_{2}} \times \cdots \times \mathbb{X}_{\lambda_{r}}
$$


Here $I_{1}, \ldots, I_{r}$ are the primary ideals with support $\lambda_{1}\left[q_{1}\right], \ldots, \lambda_{r}\left[q_{r}\right]$ such that $I_{1} \cap \cdots \cap I_{r}=I$. It follows that

$$
\begin{aligned}
\left(\mathcal{P}_{1}\right)_{I}=\left(\rho_{*} \mathcal{O}_{\mathbb{X}_{n}}\right)_{I}=\bigoplus\left(\mathcal{O}_{\mathbb{X}_{n}}\right)_{\left(I, p_{1}, \ldots, p_{n}\right)} & =\bigoplus\left(\mathcal{O}_{\mathbb{X}_{\lambda_{1}}}\right)_{\left(I_{1}, q_{1}, \ldots, q_{1}\right)} \otimes \cdots \otimes\left(\mathcal{O}_{\mathbb{X}_{\lambda_{r}}}\right)_{\left(I_{r}, q_{r}, \ldots, q_{r}\right)} \\
& =\bigoplus \bigotimes\left(\mathcal{P}_{1, q_{j}}\left(\lambda_{j}\right)\right)_{I_{j}},
\end{aligned}
$$

where the summations are over all distinct orderings of $p_{1}, \ldots, p_{n}$. As the stabiliser of $\left(p_{1}, \ldots, p_{n}\right) \in \mathbb{C}^{2 n}$ is isomorphic to the Young subgroup $S_{\lambda}=S_{\lambda_{1}} \times \cdots \times S_{\lambda_{r}}$, there are $n ! /\left|S_{\lambda}\right|$ different orderings. Hence (6.7.1) implies that

$$
\operatorname{mult}_{Z_{\lambda}} \overline{\mathcal{P}}(n)=\operatorname{mult}_{I} \overline{\mathcal{P}}_{1,0}(n)=\frac{n !}{\left|S_{\lambda}\right|} \prod_{j=1}^{r} \operatorname{mult}_{I_{j}} \overline{\mathcal{P}}_{1, q_{j}}\left(\lambda_{j}\right)
$$

where the multiplicities of the final term are taken at $I_{j}$ in $\operatorname{Hilb}^{\lambda_{j}} \mathbb{C}^{2}$.

In order to calculate the right hand side of (6.7.2), we need to assume that each $I_{j}$ is a generic point of the scheme $V_{j}=\widehat{\tau}_{\lambda_{j}}^{-1}\left(\lambda_{j}\left[q_{j}\right]\right) \subseteq$ Hilb $^{\lambda_{j}} \mathbb{C}^{2}$. We will confirm by a dimension count that this can be achieved. Since $\operatorname{dim} V_{j}=\lambda_{j}-1$, we have $\operatorname{dim}\left(V_{1} \times \cdots \times V_{r}\right)=\sum_{j=1}^{r}\left(\lambda_{j}-1\right)$. We may choose the $r$-tuple $\left(q_{1}, \ldots, q_{r}\right)$ from a non-empty open subset of $\left\{\left(q_{1}, \ldots, q_{r}\right): \sum_{j=1}^{r} \lambda_{j} q_{r}=0\right\}$. Then as we vary the $q_{j}$ 's in this parameter space the spaces $V_{1} \times \cdots \times V_{r}$ sweep out a variety of dimension $\sum_{j=1}^{r}\left(\lambda_{j}-1\right)+(r-1)=n-1=\operatorname{dim} Z_{\lambda}$. We conclude that $I$ can be chosen so that the $I_{j}$ 's are generic. It follows that $\operatorname{mult}_{I_{j}} \overline{\mathcal{P}}_{1, q_{j}}\left(\lambda_{j}\right)=$ mult $_{V_{j}} \overline{\mathcal{P}}_{1, q_{j}}\left(\lambda_{j}\right)$. Shifting the base from $q_{j}$ to 0 and writing $Z_{(m)}=\widehat{\tau}_{m}^{-1}(\mathbf{0})$ for the punctual Hilbert scheme in Hilb ${ }^{m} \mathbb{C}^{2}$ shows that

$$
\operatorname{mult}_{V_{j}} \overline{\mathcal{P}}_{1, q_{j}}\left(\lambda_{j}\right)=\operatorname{mult}_{Z_{\left(\lambda_{j}\right)}} \overline{\mathcal{P}}_{1,0}\left(\lambda_{j}\right)=\operatorname{mult}_{Z_{\left(\lambda_{j}\right)}} \overline{\mathcal{P}}\left(\lambda_{j}\right)
$$

By Lemma 6.6 this final multiplicity equals 1 . Combined with (6.7.2) this gives mult $_{Z_{\lambda}} \overline{\mathcal{P}}(n)=n ! /\left|S_{\lambda}\right|$.

Finally we compute mult $Z_{\lambda} \overline{\mathcal{P}}(n)_{\mu}$. As the dimension of geometric fibres bounds multiplicity from above, and $I_{\lambda} \in Z_{\lambda}$, (6.6.1) shows that

$$
\operatorname{mult}_{Z_{\lambda}} \overline{\mathcal{P}}(n)_{\mu} \leq \operatorname{dim} \overline{\mathcal{P}}(n)\left(I_{\lambda}\right)_{\mu}=K_{\mu \lambda} .
$$

Hence

$$
\frac{n !}{\left|S_{\lambda}\right|}=\operatorname{mult}_{Z_{\lambda}} \overline{\mathcal{P}}(n)=\sum_{\nu} \operatorname{mult}_{Z_{\lambda}} \overline{\mathcal{P}}(n)_{\nu} \operatorname{dim} \nu \leq \sum_{\nu} K_{\nu \lambda} \operatorname{dim} \nu
$$

By [Mac, Remark following I(7.8)], $\sum_{\nu} K_{\nu \lambda} \operatorname{dim} \nu=\operatorname{dim}_{\operatorname{Ind}_{S_{\lambda}}^{W}} \mathbb{C}=n ! /\left|S_{\lambda}\right|$ and so (6.7.3) is an equality for each $\mu$.

6.8. It would be interesting to know if there is a result analogous to Theorem 6.7 that holds for the simple modules $L_{c}(\mu)$, but we are unable to prove this. Indeed we do not even know whether the characteristic varieties Char $L_{c}(\mu)$ are equi-dimensional; this is a special case of of Question 4.9. The theorem does however provide information about the restricted characteristic varieties $\mathbf{r C h} L_{c}(\mu)$, as defined in (2.8.1). 
Corollary. For all $\mu \in \operatorname{Irrep}(W)$ there exist integers $a_{\lambda, \mu} \in \mathbb{N}$ such that

$$
\operatorname{rCh}\left(L_{c}(\mu)\right)=\left[Z_{\mu}\right]+\sum_{\lambda<\mu} a_{\lambda, \mu}\left[Z_{\lambda}\right]
$$

Proof. Recall from Lemma 6.4 that each $Z_{\lambda}$ has dimension $n-1$. As in (3.10), the partition $\left(1^{n}\right)$ corresponding to the sign representation of $W$ is minimal in the dominance ordering. By [GS, Remark 3.6(2)] $L_{c}$ (sign) $=\Delta_{c}$ (sign). Hence $\mathbf{r C h} L_{c}($ sign $)=\sum_{\lambda} K_{\left(1^{n}\right) \lambda}\left[Z_{\lambda}\right]=\left[Z_{\left(1^{n}\right)}\right]$, by Theorem 6.7 and (3.11). This begins an induction.

Now fix a partition $\mu$ and suppose that (6.8.1) holds for all partitions $\nu<\mu$. By [GS, Lemma 3.5 and Remark 3.5(1)], there is a short exact sequence $0 \rightarrow M \rightarrow \Delta_{c}(\mu) \rightarrow L_{c}(\mu) \rightarrow 0$ where the simple composition factors of $M$ are of the form $L_{c}(\nu)$ for $\nu<\mu$. By the inductive hypothesis and Lemma 2.8(c) $\mathbf{r C h} M=\sum_{\nu<\mu} b_{\nu}\left[Z_{\nu}\right]$ for some $b_{\nu} \in \mathbb{N}$. On the other hand, by Theorem 6.7 and $(3.11), \mathbf{r C h} \Delta_{c}(\mu)=$ $\left[Z_{\mu}\right]+\sum_{\lambda<\mu} K_{\mu \lambda}\left[Z_{\lambda}\right]$. Applying Lemma 2.8, again, shows that

$$
\operatorname{rCh} L_{c}(\mu)=\operatorname{rCh} \Delta_{c}(\mu)-\operatorname{rCh} M=\left[Z_{\mu}\right]+\sum_{\lambda<\mu} a_{\lambda, \mu}\left[Z_{\lambda}\right]
$$

for some $a_{\lambda, \mu} \in \mathbb{N}$. This completes the induction step and hence the proof of the lemma.

6.9. One case where it is easy to identify $\operatorname{rCh} L_{c}(\mu)$ is when $\mu=$ triv and $L_{c}$ (triv) is finite dimensional; thus $c=r / n$ with $(r, n)=1$ by [BEG2, Theorem 1.2]. In this case, $\operatorname{Char}_{0} L_{c}$ (triv) is the zero orbit $\mathbf{0}$ in $\mathfrak{h} \oplus \mathfrak{h}^{*} / W$ and so Proposition 4.8 shows that Char $L_{c}$ (triv) $\subseteq \tau^{-1}(\mathbf{0})=\mathrm{Z}_{\mathrm{n}}$. By Corollary 6.8 , this forces Char $L_{c}$ (triv) $=\operatorname{rCh} L_{c}($ triv $)=\left[\mathrm{Z}_{\mathrm{n}}\right]$.

6.10. Let $H_{2 n-2}(Z, \mathbb{C})$ be the top degree Borel-Moore homology group of the variety $Z$. By Lemma 6.4 and [Na, Section 8.2], the irreducible components $Z_{\lambda}$ of $Z$ form a basis of $H_{2 n-2}(Z, \mathbb{C})$, so its dimension is equal to the number of partitions of $n$.

Corollary. Taking the reduced characteristic cycle $\mathbf{r C h} M$ of a module $M \in \mathcal{O}_{c}$ induces an isomorphism of vector spaces $\mathbf{r C h}: K\left(\mathcal{O}_{c}\right) \otimes_{\mathbb{Z}} \mathbb{C} \stackrel{\sim}{\rightarrow} H_{2 n-2}(Z, \mathbb{C})$.

Proof. We first check that $\mathbf{r C h}$ is well-defined. So for any short exact sequence $0 \rightarrow M_{1} \rightarrow M_{2} \rightarrow M_{3} \rightarrow 0$ in $\mathcal{O}_{c}$, we must show that $\mathbf{r C h} M_{2}=\mathbf{r C h} M_{1}+\mathbf{r C h} M_{3}$. Since every object in $\mathcal{O}_{c}$ has a finite composition series, it is enough by Lemma 2.8(c) to prove that $\operatorname{dim} \operatorname{Char} L_{c}(\mu)=n-1$ for all $\mu \in \operatorname{Irrep}(W)$. But this is immediate from Corollary 6.8.

As $K\left(\mathcal{O}_{c}\right) \otimes_{\mathbb{Z}} \mathbb{C}$ and $H_{2 n-2}(Z, \mathbb{C})$ are vector spaces with bases $\left\{\left[L_{c}(\mu)\right]\right\}$ and $\left\{\left[Z_{\mu}\right]\right\}$, respectively, Corollary 6.8 also shows that the matrix which represents $\mathbf{r C h}$ for these choices of bases is unitriangular when we order by the dominance ordering. As such a matrix is invertible, $\mathbf{r C h}$ is an isomorphism. 
6.11. In this subsection we give a refinement of Corollary 6.10 that also justifies the comments made in (1.6) from the introduction.

According to $[\mathrm{Gr}]$ and $[\mathrm{Na},(9.13)]$ there is an isomorphism

$$
\xi: \bigoplus_{n \geq 0} H_{2 n-2}(Z(n), \mathbb{C}) \stackrel{\sim}{\rightarrow} \mathbb{C}\left[p_{k}: k \in \mathbb{N}\right]
$$

between the cohomology of the Lagrangian subvarieties $Z(n) \subseteq \operatorname{Hilb}(\mathrm{n})$ and the space of symmetric functions. Under this identification $\left[Z_{\lambda}\right]$ is sent to the monomial symmetric function $m_{\lambda}$, [Na, Corollary 9.15]. Thus by Theorem 6.7 and (3.11)

$$
\xi\left(\mathbf{r C h} \Delta_{c}(\mu)\right)=\sum_{\lambda} K_{\mu \lambda} m_{\lambda}=s_{\mu}
$$

the Schur function of $\mu$.

For generic $c, \mathcal{O}_{c}$ is semisimple and so this gives a natural description of $K\left(\mathcal{O}_{c}\right)$ in terms of symmetric functions. When $\mathcal{O}_{c}$ is not semisimple this is insufficient, and this is the case we examine here. By [BEG1, Corollary 2.11] and [Du], $\mathcal{O}_{c}$ is not semisimple if and only if $c \in \mathcal{C}$, as defined in (3.4). We fix such a value of $c$ and we assume that $\mathcal{O}_{c}$ is equivalent to $S_{q}$-mod where $S_{q}=S_{q}(n, n)$ is the $q$-Schur algebra and $q=\exp (2 \pi i c)$ is a primitive eth root of unity. As mentioned in the introduction, this is conjectured in [GGOR, Remark 5.17] and a proof has recently been announced by Rouquier.

Under this assumption, and in the notation of [GS, (3.5)], there is an equality of multiplicities

$$
\left[\Delta_{c}(\lambda): L_{c}(\tau)\right]=\left[W_{q}(\lambda): F_{q}(\tau)\right]
$$

The multiplicities on the right can be described as follows, where we refer the reader to [LT] for all definitions and notation. Let $\mathcal{F}_{v}$ be the $v$-deformed Fock space representation of $U_{v}(\widehat{\mathfrak{g} l})$, a deformation of the space of symmetric functions in an indeterminate $v$. The standard basis $\{|\lambda\rangle\}$ of $\mathcal{F}_{v}$ indexed by all partitions corresponds to the basis of symmetric functions given by the Schur functions. By [LT, Theorem 4.1], there exist upper and lower canonical bases of $\mathcal{F}_{v}$, written $\{G(\lambda)\}$ and $\left\{G^{-}(\lambda)\right\}$ respectively. If we write

$$
G(\mu)=\sum_{\lambda} d_{\lambda \mu}(q)|\lambda\rangle \quad \text { and } \quad G^{-}(\lambda)=\sum_{\mu} e_{\lambda \mu}(q)|\mu\rangle,
$$

then $d_{\lambda \mu}(q) \in \mathbb{Z}[q]$ and $e_{\lambda \mu}(q) \in \mathbb{Z}\left[q^{-1}\right]$.

If we consider only partitions of $n$, then by [LT, Section 4] these bases are related by the matrix equality

$$
\left[e_{\lambda \mu}\left(q^{-1}\right)\right]=\left[d_{\lambda^{t} \mu^{t}}(q)\right]^{-1} .
$$

The confirmation of [LT, Conjecture 5.2] in [VV] shows that $d_{\lambda^{t} \mu^{t}}(1)=\left[W_{q}(\lambda): F_{q}(\mu)\right]$. Combined with (6.11.2) and (6.11.3), this implies that $\mathbf{r C h}\left(L_{c}(\lambda)\right)=\xi^{-1}\left(\sum_{\mu} e_{\lambda \mu}(1) s_{\mu}\right)=\xi^{-1}\left(G^{-}(\lambda)_{v=1}\right)$. In other words,

Proposition. Assume that $\mathcal{O}_{c}$ is equivalent to $S_{q}$-mod and pick $\lambda \in \operatorname{Irrep}(W)$. Then the reduced characteristic cycle $\mathbf{r C h}\left(L_{c}(\lambda)\right)$ is exactly the canonical basis element $\xi^{-1}\left(G^{-}(\lambda)_{v=1}\right)$ in $H_{2 n-2}(Z, \mathbb{C})$ corresponding to the lower canonical basis element $G^{-}(\lambda)$ of the ring of symmetric functions from [LT]. 
Remark. Thanks to [VV], the polynomials $d_{\lambda \mu}(q)$ are parabolic Kazhdan-Lusztig polynomials. This hints at the possibility that the rational Cherednik algebra connects Macdonald theory with Kazhdan-Lusztig theory by providing a bridge between $q$-Schur algebras and Hilbert schemes. As an example of this, Theorem 6.7 and (6.11.2) give a number of different positive integral factorisations of the Kostka matrix in terms of evaluations of various Kazhdan-Lusztig polynomials.

6.12. An example. The results of this section are nicely illustrated by the case $n=2$ and $c=\frac{1}{2}$. (By Remark 3.4(1) all the results of this paper do hold in this case.) For these values of $n$ and $c$, [EG, Proposition 8.2] implies that $U_{c}=\mathbb{C}\left\langle x^{2}, x y, y^{2}\right\rangle \cong U\left(\mathfrak{s l}_{2}(\mathbb{C})\right) /(\Omega)$, where $\Omega$ is the Casimir element. Moreover, $M=e \Delta_{c}$ (triv) identifies with the non-simple Verma module over $U\left(\mathfrak{s l}_{2}(\mathbb{C})\right) /(\Omega)$ while $N=e \Delta_{c}(\operatorname{sign})$ embeds into $e \Delta_{c}($ triv $)$ with factor $L_{c}($ triv $) \cong \mathbb{C}$.

First consider $M$ and note that $M \cong \mathbb{C}\left[x^{2}\right]$ as a module over $\mathbb{C}\left[x^{2}\right]=\mathbb{C}[\mathfrak{h}]^{W}$. Give $M$ the induced ord filtration $\Lambda$ : thus $M=\Lambda^{0} M$. Now, $M(1)=e H_{c+1} \delta e \otimes M \cong e \Delta_{c+1}$ (triv) $\cong \mathbb{C}\left[x^{2}\right]$, by [GS, Proposition 3.16]. However, the tensor product filtration on $M(1)$ is a little subtle. Indeed, since $\delta=x$ it is clear that

$$
\Lambda^{0} M(1) \supseteq e \mathbb{C}\left[x^{2}\right] x \delta e \otimes 1=\mathbb{C}\left[x^{2}\right] x^{2},
$$

but one can check that the first occurrence of 1 in the filtration comes from $e y \delta e \otimes 1=-2 e \otimes 1$. Thus, under the tensor product filtration $\Lambda$, one has $\Lambda^{0} M(1)=\mathbb{C}\left[x^{2}\right] x^{2}$ and $\Lambda^{1} M(1) / \Lambda^{0} M(1) \cong \mathbb{C}$. We leave it to the reader to prove, by induction, that

Lemma. As above, take $n=2, c=\frac{1}{2}$ and let $M=e \Delta_{c}$ (triv) $\supset N=e \Delta_{c}$ (sign). Pick $k \geq 1$.

(1) The tensor product filtration $\Lambda$ on $M(k)=B_{c+k, c} \otimes M$ satisfies $\Lambda^{0} M(k)=\mathbb{C}\left[x^{2}\right] x^{2 k}$ and $x^{2 k-2 i} \in$ $\Lambda^{i} M(k) \backslash \Lambda^{i-1} M(k)$ for $0 \leq i \leq k$.

(2) $\operatorname{gr}_{\Lambda} \widetilde{M}=D_{1} \oplus D_{2}$, where $D_{1} \cong \mathbb{C}\left[x^{2}\right] x^{2} \oplus \mathbb{C}\left[x^{2}\right] x^{4} \oplus \cdots$ and $D_{2} \cong \mathbb{C} \otimes_{A^{0}} A$. Consequently, $\mathbf{r C h} M=$ Char $M=\mathcal{V}_{1}+\mathcal{V}_{2}$, where $\mathcal{V}_{1}=Z_{\left(1^{n}\right)}$ and $\mathcal{V}_{2}=Z_{(n)}$ is the punctual Hilbert scheme.

Note that part (2) of the lemma is in accordance with Theorem 6.7. We emphasise that $V(\operatorname{gr} \widetilde{M})$ has two components whereas $V(\operatorname{gr} \widetilde{N})$ has just one. In contrast, under the ord filtration $M$ and $N$ both have associated graded module isomorphic to $\mathbb{C}\left[x^{2}\right]$ and so $\mathrm{Char}_{0} M=\mathrm{Char}_{0} N$.

6.13. Keep the notation and hypotheses from (6.12). Proposition 5.4 and Theorem 6.7 imply that $L=$ $e L_{c}$ (triv) satisfies $\operatorname{gr}_{\Lambda} \widetilde{L}=D_{2}$ with $\mathbf{r C h} L=Z_{(n)}$, while $N=e \Delta$ (sign) satisfies $\operatorname{gr}_{\Lambda} \widetilde{N}=D_{1}$ with $\mathbf{r C h} N=$ $Z_{\left(1^{n}\right)}$. This also follows from the computations in (6.12). Indeed, give $N$ the filtration induced from that on $M$. Then $N=\mathbb{C}\left[x^{2}\right] x^{2}$ and $N(k)=e \Delta_{c+k}($ sign $)=B_{c+k, c} \otimes N=\mathbb{C}\left[x^{2}\right] x^{2 k+2}$. Repeating the argument from (6.12) then shows that $N(k)=\Lambda^{0} N(k)$ for all $k$, whence the result.

6.14. There are a couple of consequences of these computations that are worth noting. First, for a $U_{c^{-}}$-module $P$, even one in category $\mathcal{O}_{c}$, it is important to use the tensor product filtration on $\widetilde{P}$, even if the ord filtration would appear more natural. For example, suppose that one begins with $M=e \Delta_{c}$ (triv) as above and gives 
each $M(k) \cong \mathbb{C}\left[x^{2}\right]$ the ord filtration $\Gamma$; thus $\Gamma^{0} M(k)=M(k)$ for each $k$. Then $\operatorname{gr}_{\Gamma} \widetilde{M}=\mathbb{C}\left[x^{2}\right] \oplus \mathbb{C}\left[x^{2}\right] \cdots$ But this module has a different characteristic variety to the variety Char $M$ computed in (6.13). This does not contradict the earlier results since an easy variant of the proof of Lemma 2.7 can be used to show that $\operatorname{gr}_{\Gamma} \widetilde{M}$ cannot finitely generated as an $A$-module.

Second, recall from (4.3) that the tensor product and ord filtrations on the $B_{i j}$ are equal. The last paragraph shows that the analogous result fails for $M=e L_{c}$ (triv).

\section{Bimodules}

7.1. In this short section we show how to extend our results on the characteristic varieties of one-sided modules to $H_{c^{-}}$and $U_{c^{-}}$-bimodules, and we discuss the resulting characteristic varieties of Harish-Chandra bimodules. Since it requires no extra work, we will actually prove these results for $\left(U_{c}, U_{d}\right)$-bimodules and so we will assume throughout that $c, d \in \mathbb{C}$ satisfy the hypothesis of (3.4). As usual, analogues of the results proved here do hold for more general values of $c$ and $d$.

7.2. Let $\omega$ be the anti-automorphism of $D\left(\mathfrak{h}^{\text {reg }}\right) * W$ defined by $\omega(x)=x, \omega(y)=-y$ and $\omega(w)=w^{-1}$ for $x \in \mathfrak{h}^{*}, y \in \mathfrak{h}$ and $w \in W$. By [GGOR, (4.2)], $\omega$ restricts to an involutive anti-automorphism on both $H_{c}$ and $U_{c}$. Let $R \otimes S$ denote the usual external tensor product of two $\mathbb{C}$-algebras $R$ and $S$. Then we can identify the category $\left(U_{c}, U_{d}\right)$-bimod of finitely generated $\left(U_{c}, U_{d}\right)$-bimodules with the category of finitely generated left $U_{c} \otimes U_{d}$-modules: for any such bimodule $M$ and $m \in M$, we set $(p \otimes q) \cdot m=p m \omega(q)$ for $p \in U_{c}$ and $q \in U_{d}$.

We have a straightforward generalisation of Theorem 1.2.

Theorem. If $c, d$ satisfy Hypothesis 3.4 there exists a filtered $\mathbb{Z}$-algebra $C$ such that

(1) $\left(U_{c}, U_{d}\right)$-bimod is equivalent to $C$-qgr;

(2) $\operatorname{gr} C$, the associated graded ring of $C$, is isomorphic to (the $\mathbb{Z}$-algebra associated with) the homogeneous coordinate ring $\bigoplus_{k \geq 0} \mathrm{H}^{0}\left(\operatorname{Hilb}(\mathrm{n}) \times \operatorname{Hilb}(\mathrm{n}), \pi_{1}^{*} \mathcal{L}^{k} \otimes \pi_{2}^{*} \mathcal{L}^{k}\right)$, where $\pi_{j}$ denotes the projection map onto the $j^{\text {th }}$ component of $\operatorname{Hilb}(\mathrm{n}) \times \operatorname{Hilb}(\mathrm{n})$.

Proof. As above, we replace $\left(U_{c}, U_{d}\right)$-bimod by $U_{c} \otimes U_{d}$-mod. By (3.5), the $\left(U_{c+1} \otimes U_{d+1}, U_{c} \otimes U_{d}\right)$-bimodule $e H_{c+1} \delta e \otimes e H_{d+1} \delta e$ induces a Morita equivalence between $U_{c} \otimes U_{d}$-mod and $U_{c+1} \otimes U_{d+1}$-mod. Hence, defining $C$ to be the Morita $\mathbb{Z}$-algebra associated to the data

$$
\left\{U_{c+i} \otimes U_{d+i}, e H_{c+i+1} \delta e \otimes e H_{d+i+1} \delta e ; i \in \mathbb{N}\right\}
$$

and applying Lemma 2.2 gives the first part of the theorem.

In the notation of (3.5.1), $C_{i j}=B_{i j}(c) \otimes B_{i j}(d)$ for all $i \geq j \geq 0$. If we give $C$ the componentwise tensor product filtration,

$$
F^{k} C_{i j}=\sum_{r+s \leq k} \operatorname{ord}^{r} B_{i j} \otimes \operatorname{ord}^{s} B_{i j}
$$


then we deduce from [GS, Theorem 6.4(2)] that

$$
\operatorname{gr} C=\bigoplus_{i \geq j \geq 0} \operatorname{gr} B_{i j} \otimes \operatorname{gr} B_{i j} \cong \bigoplus_{i \geq j \geq 0} \mathrm{H}^{0}\left(\operatorname{Hilb}(\mathrm{n}), \mathcal{L}^{i-j}\right) \otimes \mathrm{H}^{0}\left(\operatorname{Hilb}(\mathrm{n}), \mathcal{L}^{i-j}\right) .
$$

The right hand side of this equation is the $\mathbb{Z}$-algebra associated to the graded algebra

$$
\bigoplus_{k \geq 0} \mathrm{H}^{0}\left(\operatorname{Hilb}(\mathrm{n}), \mathcal{L}^{k}\right) \otimes \mathrm{H}^{0}\left(\operatorname{Hilb}(\mathrm{n}), \mathcal{L}^{k}\right)=\bigoplus_{k \geq 0} \mathrm{H}^{0}\left(\operatorname{Hilb}(\mathrm{n}) \times \operatorname{Hilb}(\mathrm{n}), \pi_{1}^{*} \mathcal{L}^{k} \otimes \pi_{2}^{*} \mathcal{L}^{k}\right)
$$

which proves part (2) of the theorem.

7.3. Write $\left(U_{c}, U_{d}\right)$-bifilt for the category of $\left(U_{c}, U_{d}\right)$-bimodules $M$ with a good filtration $\Lambda$; by definition this means that $\left(\operatorname{ord}^{i} U_{c}\right)\left(\Lambda^{j} M\right)\left(\operatorname{ord}^{k} U_{d}\right) \subseteq \Lambda^{i+j+k} M$, for all $i, j, k$ and that the associated graded object $\operatorname{gr}_{\Lambda} M$ is finitely generated as an $\left(\operatorname{ogr} U_{c}, \operatorname{ogr} U_{d}\right)$-bimodule. Under the identification of $\left(U_{c}, U_{d}\right)$-bimod with $U_{c} \otimes U_{d}$-mod, this category is simply $U_{c} \otimes U_{d}$-filt. Thanks to Theorem 7.2, the requirements (1)-(4) of (2.3) are fulfilled, and so we have a natural functor

$$
\Upsilon^{\prime}:\left(U_{c}, U_{d}\right) \text {-bifilt } \longrightarrow \operatorname{Coh}(\operatorname{Hilb}(\mathrm{n}) \times \operatorname{Hilb}(\mathrm{n})) \quad(M, \Lambda) \mapsto \pi \operatorname{gr}_{\Lambda} M
$$

We wish to adjust $\Upsilon^{\prime}$ to take account of the twisting by $\omega$ which occurs when we pass from $\left(U_{c}, U_{d}\right)$-bimod to $U_{c} \otimes U_{d}$-mod. Note first that the ord filtration is preserved by $\omega$, so we obtain an induced action of $\omega$ on $\mathbb{C}\left[\mathfrak{h} \oplus \mathfrak{h}^{*}\right] \subset \operatorname{ogr} D\left(\mathfrak{h}^{\text {reg }}\right) * W$ given by the formulæ in (7.2). Clearly this is also induced by the automorphism $\bar{\omega}$ of $\mathfrak{h} \oplus \mathfrak{h}^{*}$ which is the identity on $\mathfrak{h}$ and multiplies $\mathfrak{h}^{*}$ by -1 . Then $\bar{\omega}$ induces automorphisms of Hilb(n) and $\mathfrak{h} \oplus \mathfrak{h}^{*} / W$ which we also denote by $\bar{\omega}$. We set

$$
\Upsilon=\left(1 \times \bar{\omega}^{*}\right) \circ \Upsilon^{\prime}:\left(U_{c}, U_{d}\right) \text {-bifilt } \longrightarrow \operatorname{Coh}(\operatorname{Hilb}(\mathrm{n}) \times \operatorname{Hilb}(\mathrm{n}))
$$

Following the discussion in Section 2, we define the characteristic variety Char $M \subseteq \operatorname{Hilb}(\mathrm{n}) \times \operatorname{Hilb}(\mathrm{n})$ of a finitely generated $\left(U_{c}, U_{d}\right)$-bimodule $M$ to be the support variety of $\Upsilon_{\Lambda} M$, for any good filtration $\Lambda$. Similarly, the associated variety $\operatorname{Char}_{0} M \subseteq\left(\mathfrak{h} \oplus \mathfrak{h}^{*}\right) / W \times\left(\mathfrak{h} \oplus \mathfrak{h}^{*}\right) / W$ is the support variety of $\left(1 \times \bar{\omega}^{*}\right)\left(\operatorname{gr}_{\Lambda} M\right)$.

7.4. Harish-Chandra bimodules. Under the canonical embeddings of $\mathbb{C}[\mathfrak{h}]^{W}$ into $U_{c}$ and $U_{d}$ any $\left(U_{c}, U_{d}\right)$ bimodule $M$ inherits the structure of a $\mathbb{C}[\mathfrak{h}]^{W}$-bimodule. Similarly, $M$ is a $\mathbb{C}\left[\mathfrak{h}^{*}\right]^{W}$-bimodule. Following [BEG1, Definition 8.8] a Harish-Chandra $\left(U_{c}, U_{d}\right)$-bimodule can therefore be defined to be a finitely generated $\left(U_{c}, U_{d}\right)$-bimodule on which the induced adjoint actions of $\mathbb{C}[\mathfrak{h}]^{W}$ and $\mathbb{C}\left[\mathfrak{h}^{*}\right]^{W}$ are locally nilpotent. We let ${ }_{c} \mathcal{H C}_{d}$ denote the category of Harish-Chandra $\left(U_{c}, U_{d}\right)$-bimodules, a full subcategory of $\left(U_{c}, U_{d}\right)$-bimod.

Proposition. If $M \in{ }_{c} \mathcal{H} \mathcal{C}_{d}$, then Char $M \subseteq \operatorname{Hilb}(\mathrm{n}) \times{ }_{\mathfrak{h} \oplus \mathfrak{h}^{*} / W} \operatorname{Hilb}(\mathrm{n})$.

Remark. The variety $\operatorname{Hilb}(\mathrm{n}) \times_{\mathfrak{h} \oplus \mathfrak{h}^{*} / W} \operatorname{Hilb}(\mathrm{n})$ is an analogue of the Steinberg variety $T^{*} \mathcal{B} \times{ }_{\mathcal{N}} T^{*} \mathcal{B}$ which features prominently in geometric representation theory. We refer the reader to $[\mathrm{BB}$, Section 3$]$ for results on the characteristic varieties of Harish-Chandra bimodules in the Lie-theoretic context. 
Proof. Set $\Omega=\left(\mathfrak{h} \oplus \mathfrak{h}^{*}\right) / W \times\left(\mathfrak{h} \oplus \mathfrak{h}^{*}\right) / W$ and let $\Gamma$ be a good filtration on $M$. We claim that the associated variety $\mathrm{Char}_{0} M$ is contained in the diagonal $\Delta$ of $\Omega$. Given the claim, Proposition 4.8 implies that

$$
\operatorname{Char} M \subseteq(\tau \times \tau)^{-1}\left(\operatorname{Char}_{0} M\right) \subseteq(\tau \times \tau)^{-1}(\Delta)=\operatorname{Hilb}(\mathrm{n}) \times_{\mathfrak{h} \oplus \mathfrak{h}^{*} / W} \operatorname{Hilb}(\mathrm{n}),
$$

where $\tau: \operatorname{Hilb}(\mathrm{n}) \rightarrow \mathfrak{h} \oplus \mathfrak{h}^{*} / W$ is the usual resolution of singularities. Thus the proposition follows.

Let $I=\operatorname{ann}_{\mathbb{C}[\Omega]}\left(\operatorname{gr}_{\Gamma} M\right)$ and write $\operatorname{Graph}(\bar{\omega})=\left\{(z, \bar{\omega}(z)): z \in \mathfrak{h} \oplus \mathfrak{h}^{*} / W\right\} \subset \Omega$. Since the definition of $\operatorname{Char}_{0}$ in (7.3) has a twist by $\bar{\omega}^{*}$, the claim is equivalent to the assertion that $\mathcal{V}(I) \subseteq \operatorname{Graph}(\bar{\omega})$. To prove this, note first that, as in [EG, Appendix D], gr $U_{c} \otimes \operatorname{gr} U_{d}$ inherits a Poisson structure from multiplication in $U_{c} \otimes U_{d}$. For $a, b, a^{\prime}, b^{\prime} \in \mathbb{C}\left[\mathfrak{h} \oplus \mathfrak{h}^{*}\right]^{W}$ this is given by

$$
\left\{a \otimes b, a^{\prime} \otimes b^{\prime}\right\}=\left\{a, a^{\prime}\right\} \otimes b b^{\prime}+a a^{\prime} \otimes\left\{b, b^{\prime}\right\},
$$

where the bracket $\{$,$\} on \mathbb{C}\left[\mathfrak{h} \oplus \mathfrak{h}^{*}\right]^{W}$ is the one arising from multiplication in either ogr $U_{c}$ or ogr $U_{d}$. (These two brackets are the same since they are both equal to the one arising from the canonical symplectic structure on $\mathfrak{h} \oplus \mathfrak{h}^{*}$.)

Since $M$ is a Harish-Chandra module, $p \otimes 1-1 \otimes \omega(p)$ acts locally nilpotently on $M$ for any element $p \in \mathbb{C}[\mathfrak{h}]^{W}$ or $p \in \mathbb{C}\left[\mathfrak{h}^{*}\right]^{W}$. We will write $\hat{p}=\sigma(p)$ for the principal symbol of $p \in U_{c}$ (or $p \in U_{d}$ ). Since $\operatorname{gr}_{\Gamma} M$ is finitely generated, on passing to associated graded modules, it follows that $\hat{p} \otimes 1-1 \otimes \omega(\hat{p}) \in \sqrt{I}$ for all homogeneous elements $\hat{p} \in \mathbb{C}[\mathfrak{h}]^{W}$ or $\hat{p} \in \mathbb{C}\left[\mathfrak{h}^{*}\right]^{W}$. As $\omega$ is an anti-automorphism of $U_{d}$ we have $\{\omega(\hat{p}), \omega(\hat{q})\}=\omega\{\hat{q}, \hat{p}\}$ for any homogeneous elements $\hat{p}, \hat{q} \in \operatorname{ogr} U_{c}=\operatorname{ogr} U_{d}$ and so

$$
\{\hat{p} \otimes 1-1 \otimes \omega(\hat{p}), \hat{q} \otimes 1-1 \otimes \omega(\hat{q})\}=\{\hat{p}, \hat{q}\} \otimes 1+1 \otimes\{\omega(\hat{p}), \omega(\hat{q})\}=\{\hat{p}, \hat{q}\} \otimes 1-1 \otimes \omega(\{\hat{p}, \hat{q}\}),
$$

for any such $\hat{p}, \hat{q}$. By Gabber's integrability theorem [Ga, Theorem 1] or $[\operatorname{Bj} 2$, Theorem 1.3], $\{\sqrt{I}, \sqrt{I}\} \subseteq \sqrt{I}$ while, by [Wa, Appendix 2], $\mathbb{C}\left[\mathfrak{h} \oplus \mathfrak{h}^{*}\right]^{W}$ is generated by the homogeneous elements in $\mathbb{C}[\mathfrak{h}]^{W}, \mathbb{C}[\mathfrak{h} *]^{W}$ and their iterated Poisson brackets. The displayed equation therefore implies that $\sqrt{I}$ contains $r \otimes 1-1 \otimes \omega(r)$ for all $r \in \mathbb{C}\left[\mathfrak{h} \oplus \mathfrak{h}^{*}\right]^{W}$. In other words, $\mathcal{V}(I) \subseteq \operatorname{Graph}(\bar{\omega})$. This completes the proof of the claim, and hence of the proposition.

7.5. The obvious analogue of Proposition 7.4 also holds for the category of Harish-Chandra $\left(H_{c}, H_{d}\right)$ bimodules. The reason is that, thanks to the natural analogue of [GS, Theorem 3.3], the idempotent functor which sends $M \in\left(H_{c}, H_{d}\right)$-bimod to $e M e \in\left(U_{c}, U_{d}\right)$-bimod is an equivalence of categories.

7.6. Questions. We end this section with several questions and comments about Harish-Chandra modules.

Let $Y$ be the variety Hilb(n) $\times_{\mathfrak{h} \oplus \mathfrak{h}^{*} / W}$ Hilb(n) introduced in Proposition 7.4. It follows from the proof of Theorem 4.10 that the resolution of singularities $\tau: \operatorname{Hilb}(\mathrm{n}) \rightarrow \mathfrak{h} \oplus \mathfrak{h}^{*} / W$ is semismall in the sense of [Na, Definition 6.7]. Following [Na, Section 6.2] we can make this more precise. First, $\mathfrak{h} \oplus \mathfrak{h}^{*} / W$ is stratified as in (6.4) by the locally closed subvarieties $S_{\lambda}=\left(\mathfrak{h} \oplus \mathfrak{h}^{*} / W\right) \cap S_{\lambda} \mathbb{C}^{2}$ for $\lambda$ a partition of $n$. The resolution 
$\tau$ then restricts to a topological fibre bundle $\tau: \tau^{-1}\left(S_{\lambda}\right) \rightarrow S_{\lambda}$ with fibre equal to the product of punctual Hilbert schemes $Z_{\left(\lambda_{1}\right)} \times \cdots \times Z_{\left(\lambda_{r}\right)}$, where $\lambda=\left(\lambda_{1}, \ldots, \lambda_{r}\right)$ has exactly $r$ nonzero parts. If we set

$$
Y_{\lambda}=\left\{\left(z, z^{\prime}\right) \in Y: \tau(z)=\tau\left(z^{\prime}\right) \in S_{\lambda}\right\}
$$

then $Y=\bigsqcup_{\lambda} Y_{\lambda}$ and $\operatorname{dim} Y_{\lambda}=(2 r-2)+(n-r)+(n-r)=2(n-1)$ for each $\lambda$ with $r$ nonzero parts. Thus $Y$ is pure of dimension $2(n-1)$ with irreducible components equal to the closures of the various $Y_{\lambda}$.

This raises two questions:

(1) Does every Harish-Chandra bimodule have (restricted) characteristic cycle equal to a weighted sum of the $Y_{\lambda}$ ?

(2) If so, what are those weights for important Harish-Chandra bimodules, for example the bimodules $B_{i j}(c)$ from (3.5.1)? Further examples of Harish-Chandra bimodules appear in [BEG1, Section 8].

7.7. Recall from [CG, Section 2.7] the convolution product on Borel-Moore homology.

Lemma. Under the convolution product there is an algebra isomorphism

$$
\Xi: H_{4(n-1)}(Y, \mathbb{C}) \cong \operatorname{Rep}(W) \otimes_{\mathbb{Z}} \mathbb{C},
$$

where $\operatorname{Rep}(W)$ is the representation ring of $W$.

Proof. This follows immediately from the formalism of [CG, Section 8.9]. Indeed, let $x \in S_{\lambda}$. Since $\tau^{-1}(x)$ is irreducible, the local system on $S_{\lambda}$ associated to $H_{2(n-r)}\left(\tau^{-1}(x), \mathbb{C}\right)$ is trivial. Applying [CG, Corollary 8.9.8 and Proposition 8.9.9(b)] shows that $H_{4(n-1)}(Y, \mathbb{C})$ is a commutative semisimple $\mathbb{C}$-algebra with basis labelled by partitions of $n$. Since $\operatorname{Rep}(W) \otimes_{\mathbb{Z}} \mathbb{C}$ has a non-degenerate associative bilinear form given by the inner product on characters is non-degenerate, it is semisimple, and so the lemma follows.

A solution to the next problem would provide a significant refinement of the lemma.

Problem. Identify the $\mathbb{T}^{2}$-equivariant Grothendieck group $K^{\mathbb{T}^{2}}(Y)$ under the convolution product.

7.8. When $c=d$, write ${ }_{c} \mathcal{H C}_{d}=\mathcal{H C}_{c}$. It is known that $\mathcal{H C}_{c}$ is monoidal under tensoring over $U_{c}$, and that $\mathcal{H C}_{c}$ is equivalent to $\mathbb{C} W$-mod as a monoidal category for $c \in \mathbb{N}$, [BEG1, Lemma 8.3 and Theorem 8.5]. It acts on $\mathcal{O}_{c}$ by tensoring over $H_{c}$.

Question. Is there a ring isomorphism $\Theta: K\left(\mathcal{H C}_{c}\right) \longrightarrow \operatorname{Rep}(W)$ such that

(1) the following diagram commutes.

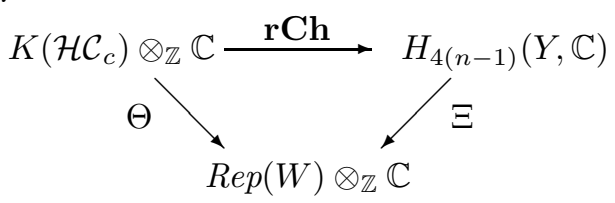

(2) $K\left(\mathcal{O}_{c}\right)$ corresponds to the regular representation under the map sending $\left[\Delta_{c}(\lambda)\right]$ to $[\lambda]$ ? 
Implicit in these questions is the assertion that the simple objects of $\mathcal{H C}_{c}$ can be labelled by partitions of $n$; once again, this is known only for $c \in \mathbb{N}$ where it follows from the equivalence of monoidal categories $\mathcal{H C}_{c} \simeq \mathbb{C} W$-mod. One can ask: Does such an equivalence exists for all $c \notin \mathcal{C}$.

If part (1) of the question is true, then Question 7.6(1) would follow for simple and hence all HarishChandra modules.

\section{A Conze Embedding}

8.1. Let $Q$ be a minimal primitive ideal in the enveloping algebra $U(\mathfrak{g})$ of a semisimple complex Lie algebra g. A classic result of Conze $[\mathrm{Co}]$ shows that $U(\mathfrak{g}) / Q$ embeds in a Weyl algebra $A_{m}=D\left(\mathbb{C}^{m}\right)$ in such a way that the restriction to $U(\mathfrak{g}) / Q$ of the canonical $D\left(\mathbb{C}^{m}\right)$-module $\mathcal{O}\left(\mathbb{C}^{m}\right)$ is a Verma module. Moreover, $D\left(\mathbb{C}^{m}\right)$ is a union of Harish-Chandra bimodules $P_{i}^{\prime}$ coming from the translation principle [JS, Proposition 5.5].

The main result of this section shows that the natural analogue of this result holds for $U_{c}$. Indeed, as we show, $U_{c} \hookrightarrow D(\mathfrak{h} / W)=\bigcup P_{i}$, where the $P_{i}$ are the natural analogues of the Harish-Chandra bimodules $P_{i}^{\prime}$ : they are the duals of the progenerative bimodules $B_{i 0}(c)$. In particular, $D(\mathfrak{h} / W)$ is a flat left $U_{c}$-module. As might be expected from the earlier sections, this result also corresponds to a basic fact about Hilb(n). In the notation of [Ha1] we consider the principal affine cell $V=\mathcal{O}_{\left(1^{n}\right)}$ in $\operatorname{Hilb}(\mathrm{n})$ corresponding to the partition $\left(1^{n}\right)$. It follows from [Ha1, Corollaries 2.7 and 2.8] that $V \cong \mathbb{C}^{2 n-2}$ is the open set $\mathcal{D}(\delta)=\operatorname{Hilb}(\mathrm{n}) \backslash \mathcal{V}(\delta)$ complementary to the zero set of $\delta$. As we show, ogr $D(\mathfrak{h} / W)=\bigcup \delta^{-i} A^{i}=\mathcal{O}(V)$.

8.2. We now turn to the proofs of these assertions. Pick $c \in \mathbb{C}$ satisfying Hypothesis 3.4.

For $i \in \mathbb{N}$ let $P_{i+1}^{i}=\delta^{-1} e_{-} H_{c+i+1} e$ and notice that, by (3.5), this is the dual of the progenerative $\left(U_{c+i+1}, U_{c+i}\right)$-bimodule $B_{i+1, i}(c)$. Thus, for $i>j \geq 0$,

$$
P_{i}^{j}=P_{j+1}^{j} P_{j+2}^{j+1} \cdots P_{i}^{i-1}=\left(B_{i j}(c)\right)^{*}
$$

is a progenerative $\left(U_{c+j}, U_{c+i}\right)$-bimodule. By [GS, Lemma 6.11(1)], $B_{i j}(c) \subseteq U_{c+i} \cap U_{c+i+1}$ and so, by induction, $U_{c} \subseteq P_{i}^{0} \subseteq P_{i+1}^{0}$, for all $i \geq 1$.

8.3. Lemma. For all $\ell \geq 1, P_{\ell}^{0} \subseteq D(\mathfrak{h} / W)$.

Proof. Pick $i \geq 0$. The algebra $H_{c+i+1}$ acts naturally on $\mathbb{C}[\mathfrak{h}]$ (see the proof of [EG, Proposition 4.5]) and so $P_{i+1}^{i}=\delta^{-1} e_{-} H_{c+i+1} e$ acts naturally on $\mathbb{C}\left[\mathfrak{h}^{\mathrm{reg}}\right]$. It is important to notice that (3.3.1) is an equality in $D\left(\mathfrak{h}^{\text {reg }}\right) * W$ and so the action of $H_{c+i}$ on $\mathbb{C}\left[\mathfrak{h}^{\text {reg }}\right]$ induced from that of $P_{i+1}^{i}$ does equal the action coming from the inclusion $H_{c+i} \subseteq D\left(\mathfrak{h}^{\text {reg }}\right) * W$.

In fact, the action of $P_{i+1}^{i}$ on $\mathbb{C}\left[\mathfrak{h}^{\text {reg }}\right]$ restricts to an action on $\mathbb{C}[\mathfrak{h}]^{W}=\mathbb{C}[\mathfrak{h} / W]$. To see this note that, for $p \in H_{c+i+1}$, the element $e_{-}$pe maps $\mathbb{C}[\mathfrak{h}]^{W}$ to $e_{-} \mathbb{C}[\mathfrak{h}]=\mathbb{C}[\mathfrak{h}]^{\text {sign }}$. Since $\mathbb{C}[\mathfrak{h}]^{\text {sign }}=\delta \mathbb{C}[\mathfrak{h}]^{W}$, we conclude that $\delta^{-1} e_{-}$pe maps $\mathbb{C}[\mathfrak{h}]^{W}$ to itself.

It remains to check that elements $\delta^{-1} e_{-} p e \in P_{i+1}^{i}$ are actually differential operators on $\mathbb{C}[\mathfrak{h}]^{W}$. But if $q \in \mathbb{C}[\mathfrak{h}]^{W}$ then $\left[q, \delta^{-1} e_{-} p e\right]=\delta^{-1} e_{-}[q, p] e$. By $\left[\right.$ BEG1, Lemma 3.3(v)] $\mathbb{C}[\mathfrak{h}]^{W}$ acts ad-nilpotently on $H_{c+i}$ 
and so $q$ also acts ad-nilpotently on $\delta^{-1} e_{-} p e \in P_{i+1}^{i}$. By definition, this means that $P_{i+1}^{i} \subseteq D(\mathfrak{h} / W)$. By induction, the same is true for the multiples $P_{i}^{j}$ of these spaces.

8.4. We next want to understand $\bigcup \delta^{-i} A^{i}$. To begin, consider $\operatorname{Hilb}^{\mathrm{n}} \mathbb{C}^{2}$ and the corresponding union $\bigcup_{i} \delta^{-i} \mathbb{A}^{i}$, where $\mathbb{A}^{0}=\mathbb{C}\left[\mathbb{C}^{2 n}\right]^{W}, \mathbb{A}^{1}=\mathbb{C}\left[\mathbb{C}^{2 n}\right]^{\text {sign }}$, and $\mathbb{A}^{i}=\left(\mathbb{A}^{1}\right)^{i}$ in the notation of $[\mathrm{GS}$, (4.3)]. We bigrade $\mathbb{A}^{0}$ and hence the $\mathbb{A}^{i}$ by the Euler and order gradations; thus $\mathbb{C}\left[\mathbb{C}^{2 n}\right]=\mathbb{C}\left[u_{1}, \ldots, u_{n}, v_{1}, \ldots, v_{n}\right]$ is graded by $\operatorname{bideg}\left(u_{i}\right)=(1,0)$ while $\operatorname{bideg}\left(v_{j}\right)=(-1,1)$, and we have chosen our variables so that $W$ acts by permutation of the subscripts.

In the notation of $\left[\mathrm{Ha1}\right.$, Section 2] we want to consider the affine open cell $U=U_{\left(1^{n}\right)} \subset \mathrm{Hilb}^{\mathrm{n}} \mathbb{C}^{2}$. By [Ha1, Corollary 2.7], $\mathcal{O}(U)$ is generated by elements $\Delta_{D} \Delta_{\left(1^{n}\right)}^{-1}$ where, by [Ha1, $(2.22)$ and the paragraph after $(2.32)]$, the $\Delta_{D}$ are a basis of $\mathbb{A}^{1}$ and $\Delta_{\left(1^{n}\right)}=\delta$. Thus $\mathcal{O}(U)=\bigcup \delta^{-i} \mathbb{A}^{i}=\mathbb{C}\left[\delta^{-1} \mathbb{A}^{1}\right]$. Moreover, by $[$ Ha1, Corollary 2.8], $\mathcal{O}_{U}=\mathcal{O}(U)$ is a polynomial $\operatorname{ring} R=\mathbb{C}\left[e_{1}, \ldots e_{n}, a_{1}, \ldots, a_{n}\right]$ (where our indexing differs by one from Haiman's). By [Ha1], the $e_{i}$ 's are the elementary symmetric functions in the $u_{j}$ 's and so (possibly after reordering $) \operatorname{bideg}\left(e_{j}\right)=(j, 0)$ for each $j$. Note that $e_{1}=\sum u_{i}=\mathbf{z}$, in the notation of (3.6). The $a_{j}$ are the coefficients of the Lagrange interpolation polynomial $\phi_{a}(x)=\sum_{i=1}^{n} a_{i} x^{i-1}=\sum_{j} v_{j} \prod_{k \neq j}\left(x-u_{k}\right)\left(u_{j}-u_{k}\right)^{-1}$. Since this polynomial is homogeneous as a polynomial in either the $v$ 's or $u$ 's, with total degrees 1 in the $v$ 's and 0 in the $u$ 's, it follows that $\operatorname{bideg}\left(a_{j}\right)=(-j, 1)$ for each $j$. In this case it is not easy to express the element $\mathbf{z}^{*}=\sum v_{i}$ from (3.6) in terms of these variables, but at least $\operatorname{bideg}\left(\mathbf{z}^{*}\right)=(-1,1)=\operatorname{bideg}\left(a_{1}\right)$.

Now consider Hilb(n). By [GS, Lemma 4.9(1)] and the comments of the last paragraph, $A^{0}=\mathbb{A}^{0} /\left(e_{1}, \mathbf{z}^{*}\right)$. Therefore,

$$
\bigcup_{i} \delta^{-i} A^{i}=\mathbb{C}\left[\delta^{-1} A^{1}\right]=\mathbb{C}\left[\delta^{-1} \mathbb{A}^{1}\right] /\left(e_{1}, \mathbf{z}^{*}\right)=R /\left(e_{1}, \mathbf{z}^{*}\right) .
$$

Since $S=R /\left(e_{1}\right)$ is a domain, the element $\mathbf{z}^{*}$ is a regular element in $S$ of the same bidegree as the element $a_{1}$. Thus the factor rings $\mathbb{C}\left[\delta^{-1} A^{1}\right]=S /\left(\mathbf{z}^{*}\right)$ and $S /\left(a_{1}\right)$ have the same bigraded Poincaré series. On the other hand, the restriction of $\mathcal{O}_{U}$ to $\operatorname{Hilb}(\mathrm{n})$ is clearly just the open subvariety $\mathcal{D}(\delta)$ complementary to $\mathcal{V}(\delta)$ in $\operatorname{Hilb}(\mathrm{n})$. Combining these observations gives:

Lemma. $\mathbb{C}\left[\delta^{-1} A^{1}\right]=\bigcup \delta^{-i} A^{i}=\mathcal{O}(V)$, where $V=\mathcal{D}(\delta)$. Also, $\mathbb{C}\left[\delta^{-1} A^{1}\right]$ has the same bigraded Poincaré series as the polynomial ring $\mathbb{C}\left[e_{2}, \ldots e_{n}, a_{2}, \ldots, a_{n}\right]$, where bideg $\left(e_{i}\right)=(i, 0)$ and bideg $\left(a_{i}\right)=(-i, 1)$.

8.5. We can now prove our analogue of the Conze embedding.

Proposition. Assume that $c \in \mathbb{C}$ satisfies Hypothesis 3.4. Then $D(\mathfrak{h} / W)=\bigcup_{i \geq 0} P_{i}^{0}$. Moreover,

$$
\operatorname{ogr} D(\mathfrak{h} / W)=\bigcup \operatorname{ogr} P_{i}^{0}=\mathbb{C}\left[\delta^{-1} A^{1}\right]=\mathcal{O}(V)
$$

where $V \cong \mathbb{C}^{2 n-2}$ is the open affine subvariety $\mathcal{D}(\delta)$ of $\operatorname{Hilb}(\mathrm{n})$.

Proof. By (3.5) the $P_{i}^{0}$ are progenerators for $U_{c}$. Give $P_{i}^{0}$ the induced filtration ord arising from the order filtration ord on $D\left(\mathfrak{h}^{\text {reg }}\right) * W$. There is a potential ambiguity, here, since $P_{i}^{0}$ can also be given an order filtration 
induced from the order filtration on $D(\mathfrak{h} / W)$. However, thanks to [Le, Lemma 3.2.1 and Proposition 3.5] the restriction map $D\left(\mathfrak{h}^{\text {reg }}\right)^{W} \rightarrow D\left(\mathfrak{h}^{\text {reg }} / W\right)$ is a filtered isomorphism and so the two filtrations agree. In particular, ogr $P_{i}^{0} \subseteq \operatorname{gr} D(\mathfrak{h} / W)$.

If $j \geq 0$, then [GS, Lemma 6.8] shows that

$$
\operatorname{ogr} P_{j+1}^{j}=\delta^{-1} \operatorname{ogr}\left(e_{-} H_{c+j+1} e\right)=\delta^{-1} e_{-}\left(\mathbb{C}\left[\mathfrak{h} \oplus \mathfrak{h}^{*} * W\right) e=\Theta\left(\delta^{-1} A^{1}\right),\right.
$$

where $\Theta: \mathbb{C}\left[\mathfrak{h}^{\text {reg }} \oplus \mathfrak{h}^{*}\right]^{W} \rightarrow e\left(\mathbb{C}\left[\mathfrak{h}^{\text {reg }} \oplus \mathfrak{h}^{*}\right]^{W}\right) e$ is the isomorphism given by $\Theta(f)=e f e$. Therefore, by induction and [GS, Lemma 6.7]

$$
\Theta\left(\delta^{-i} A^{i}\right)=\Theta\left(\left(\delta^{-1} A^{1}\right)^{i}\right)=\left(\operatorname{ogr} P_{1}^{0}\right)\left(\operatorname{ogr} P_{2}^{1}\right) \cdots\left(\operatorname{ogr} P_{i}^{i-1}\right) \subseteq \operatorname{ogr} P_{i}^{0}
$$

and so, by Lemma 8.3,

$$
\mathbb{C}\left[\delta^{-1} A^{1}\right]=\bigcup_{i \geq 0} \delta^{-i} A^{i} \text { is isomorphic to a subspace of } \bigcup_{i \geq 0} \operatorname{ogr} P_{i}^{0} \subseteq \operatorname{ogr} D(\mathfrak{h} / W) .
$$

Clearly each $P_{i}^{0}$ is graded under the E-grading and so gr $P_{i}^{0}$ has an induced (E, ord) bigrading, as in (8.4). Now $\mathbb{C}[\mathfrak{h}]^{W}=\mathbb{C}\left[e_{2}, \ldots, e_{n}\right]$ is a polynomial ring whose generators have degree and hence $\mathbf{E}$-degree E- $\operatorname{deg}\left(e_{i}\right)=i$. Since the equation $\partial_{e_{i}} e_{i}-e_{i} \partial_{e_{i}}=1$ in $D(\mathfrak{h} / W)$ is E-homogeneous, E-deg $\partial_{e_{i}}=-i$ for each $i$. Therefore $\operatorname{ogr} D(\mathfrak{h} / W)=\mathbb{C}\left[e_{2}, \ldots, e_{n}, d_{2}, \ldots, d_{n}\right]$ is a polynomial ring whose generators satisfy $\operatorname{bideg} e_{i}=(i, 0)$ and bideg $d_{i}=(-i, 1)$.

By (8.5.1) and Lemma 8.4, $\mathbb{C}\left[\delta^{-1} A^{1}\right]$ is therefore a bigraded algebra with exactly the same bigraded Poincaré series as that of its overring $\operatorname{ogr} D(\mathfrak{h} / W)$. Since the bigraded components of these algebras are finite dimensional, they must be equal. In particular, (8.5.1) implies that $\bigcup_{i \geq 0} \operatorname{ogr} P_{i}^{0}=\operatorname{ogr} D(\mathfrak{h} / W)$ and hence that $\bigcup_{i \geq 0} P_{i}^{0}=D(\mathfrak{h} / W)$. Finally, as $\operatorname{ogr} D(\mathfrak{h} / W)$ is a polynomial ring, this also implies that $V=$ $\operatorname{Spec}\left(\mathbb{C}\left[\delta^{-1} A^{1}\right]\right)$ is isomorphic to $\mathbb{C}^{2 n-2}$.

8.6. Since the $P_{i}^{0}$ are projective left $U_{c}$-modules we obtain:

Corollary. Keep the hypotheses and notation of Proposition 8.5. Then $D(\mathfrak{h} / W)$ is a flat left $U_{c}$-module.

We remark that $D(\mathfrak{h} / W)$ will typically not be a flat right module over $U_{c}$. Indeed this even fails for $n=2$, when $U_{c}$ is a primitive factor ring of $U\left(\mathfrak{s l}_{2}\right)$ (see [JS, (5.7)]).

\section{INDEX OF NotATION}

$A^{1}$, alternating polynomials, $A=\bigoplus A^{k} \delta^{k}$,

$\widehat{A}=\bigoplus_{i \geq j \geq 0} A^{i-j} \delta^{i-j}$,

$\mathcal{B}$, the tautological rank $n$ bundle,

$B=\oplus B_{i j}$,

$\mathcal{C}$, the set of singular values for $c$,

$\widetilde{C}, \widetilde{C}_{\lambda}$,

Char, characteristic varieties,
$\mathrm{Char}_{0}$, associated varieties,

$\mathbf{C h}$, characteristic cycle,

rCh, restricted characteristic cycle,

$d_{\mu}=\left\{(i, j) \in \mathbb{N} \times \mathbb{N}: j<\mu_{i+1}\right\}$,

$\delta=\prod_{s \in \mathcal{S}} \alpha_{s}$,

$\Delta_{c}(\tau)$, the standard module, dominance ordering on $\operatorname{Irrep}(W)$,
(2.6),(7.3) 
Euler operator $\mathbf{E}=\sum x_{i} \delta_{i}, \mathbf{E}-\mathrm{deg}$, $e, e_{-}$, trivial and sign idempotents,

fake degrees $f_{\tau}$,

good filtrations,

$H_{c}$, the rational Cherednik algebra, $\mathfrak{h}, \mathfrak{h}^{*}$,

Hilbert schemes $\operatorname{Hilb}^{\mathrm{n}} \mathbb{C}^{2}$, Hilb(n),

$I_{\mu}$, monomial ideal for partition $\mu$,

Irrep $(W)$, the irred. reps. of $W$,

$J^{1}=\mathbb{C}\left[\mathfrak{h} \oplus \mathfrak{h}^{*}\right] A^{1}$,

$K_{\mu \lambda}(t, s)$, Kostka-Macdonald coefficients,

$L_{c}(\tau)$, simple $\mathcal{O}_{c}$-module,

$\mathcal{L}=\bigwedge^{n} \mathcal{B} \cong \mathcal{O}_{\text {Hilb(n) }}(1)$,

$\mathcal{O}_{c}$, category $\mathcal{O}$ for $H_{c}$,

ord, ogr, order filtration and gradation,

$\mathcal{P}=\rho_{*} \mathcal{O}_{X_{n}}$, the Procesi bundle,

$\mathcal{P}_{\mu}, V_{\mu}$,
$\Phi, \widehat{\Phi}$,

Poincaré series $p(M, s, t), p(M, v)$,

qgr, Qgr,

$\rho: X_{n} \rightarrow \operatorname{Hilb}^{\mathrm{n}} \mathbb{C}^{2}$,

$\sigma(m)$, the principal symbol of $m$,

sign, the sign representation of $W$,

$\mathbb{T}^{2}$-action

$\widehat{\tau}: \operatorname{Hilb}^{\mathrm{n}} \mathbb{C}^{2} \rightarrow \mathbb{C}^{2 n} / W$,

$\tau: \operatorname{Hilb}(\mathrm{n}) \rightarrow \mathfrak{h} \oplus \mathfrak{h}^{*} / W$,

tot, tgr, total degree filtration/gradation

triv, the trivial $W$-representation,

$U_{c}=e H_{c} e$, the spherical subalgebra,

$Z=Z(n)=\sigma^{-1}(\mathfrak{h} / W), Z_{\lambda}$,

$\mathrm{Z}_{\mathrm{n}}=Z_{(n)}$, the punctual Hilbert scheme,

\section{REFERENCES}

[BEG1] Y. Berest, P. Etingof and V. Ginzburg, Cherednik algebras and differential operators on quasi-invariants, Duke Math. J., 118 (2003), 279-337.

[BEG2] Y. Berest, P. Etingof and V. Ginzburg, Finite dimensional representations of rational Cherednik algebras, Int. Math. Res. Not., 2003, No. 19, 1053-1088.

[Bj1] J.-E. Björk, Rings of Differential Operators, North-Holland Mathematical Library Vol 21, North-Holland, Amsterdam, 1979.

[Bj2] J.-E. Bjork, Filtered noetherian rings, in Noetherian Rings and their applications, Ed. L. W. Small, Math Surveys and Monographs Vol. 24, Amer. Math. Soc., Providence, RI.

[BB] W. Borho and J.-L. Brylinski, Differential operators on homogeneous spaces, III, Characteristic varieties of HarishChandra modules and of primitive ideals, Inventiones Math., 80 (1982), 1-68.

[BKR] T. Bridgeland, A. King and M. Reid, The McKay correspondence as an equivalence of derived categories, J. Amer. Math. Soc., 14 (2001), 535-554.

[CG] N. Chriss and V. Ginzburg, Representation Theory and Complex Geometry, Birkhäuser (1997).

[Co] N. Conze, Algèbres d'opérateurs différentiels et quotients des algèbres enveloppantes, Bull. Soc. Math. France, 102 (1974), 379-415.

[De] C. Dezélée, Représentations de dimension finie de l'algèbre de Cherednik rationnelle, Bull. Soc. Math. France, 131 (2003), 465-482.

[Du] C. F. Dunkl, Singular polynomials for the symmetric groups, to appear, math.RT/0403277.

[EG] P. Etingof and V. Ginzburg, Symplectic reflection algebras, Calogero-Moser space, and deformed Harish-Chandra homomorphism, Invent. Math., 147 (2002), 243-348.

[Ga] O. Gabber, The integrability of the characteristic variety, Amer. J. Math., 103 (1981), 445-468.

[GG] W. L. Gan and V. Ginzburg, Almost commuting varieties, D-modules and Cherednik algebras, to appear, math.RT/0409262.

[GH1] A. M. Garsia and M. Haiman, A remarkable $q, t$-Catalan sequence and $q$-Lagrange inversion, J. Algebra. Combin., 5 (1996), 191-244. 
[GH2] A. M. Garsia and M. Haiman, Some natural bigraded $S_{n}$-modules and $q, t$-Kostka coefficients. The Foata Festschrift, Electron. J. Combin., 3 (1996), no. 2, Research Paper 24 (electronic).

[GGOR] V. Ginzburg, N. Guay, E. Opdam and R. Rouquier, On the category $\mathcal{O}$ for rational Cherednik algebras, Invent. Math., 154 (2003), 617-651.

[G2] I. Gordon, On the quotient by diagonal invariants, Invent. Math., 153 (2003), 503-518.

[GS] I. Gordon and J. T. Stafford, Rational Cherednik algebras and Hilbert schemes, to appear, math.RA/0407516.

[Gr] I. Grojnowski, Instantons and affine algebras I: the Hilbert scheme and vertex operators, Int Math. Res. Lett., 3 (1996), no.2, 275-291.

[EGA] A. Grothendieck, Éléments de géométrie algébrique II, Publ. Math. IHES 8 (1961).

$[\mathrm{Gu}] \quad$ N. Guay, Projective modules in the category $\mathcal{O}$ for the Cherednik algebra, J. Pure and Appl. Algebra, 182 (2003), 209-221.

[Ha1] M. Haiman, $t, q$-Catalan numbers and the Hilbert scheme, Discrete Math., 198 (1998), 201-224.

[Ha2] M. Haiman, Hilbert schemes, polygraphs and the Macdonald positivity conjecture, J. Amer. Math. Soc., 14 (2001), 941-1006.

[Ha3] M. Haiman, Vanishing theorems and character formulas for the Hilbert scheme of points in the plane, Invent. Math., 149 (2002), 371-407.

[JS] A. Joseph and J. T. Stafford, Modules of $\mathfrak{k}$-finite vectors over semisimple Lie algebras, Proc. London Math. Soc., 49 (1984), 361-384.

[KL] G. Krause and T. Lenagan, Growth of algebras and Gelfand-Kirillov dimension, Revised edition, Graduate Studies in Math. 22, Amer. Math. Soc., Providence, RI, 2000.

[LT] B. Leclerc and J.-Y. Thibon, Canonical bases of $q$-deformed Fock spaces, Int. Math. Res. Notices, 9 (1996), $447-456$.

[Le] T. Levasseur, Relèvements d'opérateurs différentiels, in Operator Algebras, Unitary Representations, Enveloping Algebras, and Invariant Theory, Ed. A. Connes et al, Prog. Math. 92, (1990) Birkhäuser, Boston 1990.

[MR] J. C. McConnell and J. C. Robson, Noncommutative Noetherian Rings, Grad. Texts in Math. 30, Amer. Math. Soc., Providence, RI, 2000.

[Mac] I. G. Macdonald, Symmetric Functions and Hall Polynomials, Second Edition, Oxford Math. Monographs, OUP, Oxford, 1995.

[Na] H. Nakajima, Lectures on Hilbert Schemes of Points on Surfaces, Univ. Lect. Series Vol. 18, AMS, Providence, RI, 1999.

[VV] M. Varagnolo and E. Vasserot, On the decomposition matrices of the quantized Schur algebra, Duke Math. J., 100 (1999), 267-297.

[Wa] N. Wallach, Invariant differential operators on a reductive Lie algebra and Weyl group representations, J. Amer. Math. Soc., 6 (1993), 779-816.

[Wt] K. Watanabe, Certain invariant subrings are Gorenstein, I, Osaka J. Math., 11 (1974), 1-8.

Department of Mathematics, Glasgow University, Glasgow G12 8QW, Scotland

E-mail address: ig@maths.gla.ac.uk

Department of Mathematics, University of Michigan, Ann Arbor, Mi 48109-1109, USA.

E-mail address: jts@umich.edu 